\title{
A Homologation Strategy for the Generation of 1-Chloroalkyl Radicals from Organoboranes
}

\author{
Gong Xu, Monique Lüthy, Jacqueline Habegger, and Philippe Renaud* \\ University of Bern, Department of Chemistry and Biochemistry, Freiestrasse 3, CH-3012 \\ Bern \\ philippe.renaud@dcb.unibe.ch
}

\section{Supporting Information}
${ }^{1} \mathrm{H}$ - and ${ }^{13} \mathrm{C}-\mathrm{NMR}$ spectra of compounds $\mathbf{4 a}-\mathbf{5 2}$
p. 2-89
Ee determination of $(R)$-1-(4-chlorophenxl)ethanol p. 90
Ee determination of $\mathbf{3 6}$
p. 91
Ee determination of $\mathbf{4 8}$
p. 92
$\mathrm{X}$-Ray crystal structure of syn-37
p. $93-95$
X-Ray crystal structure of $\mathbf{4 4}$
p. $96-98$
X-Ray crystal structure of $\mathbf{4 8}$
p. 99-101 


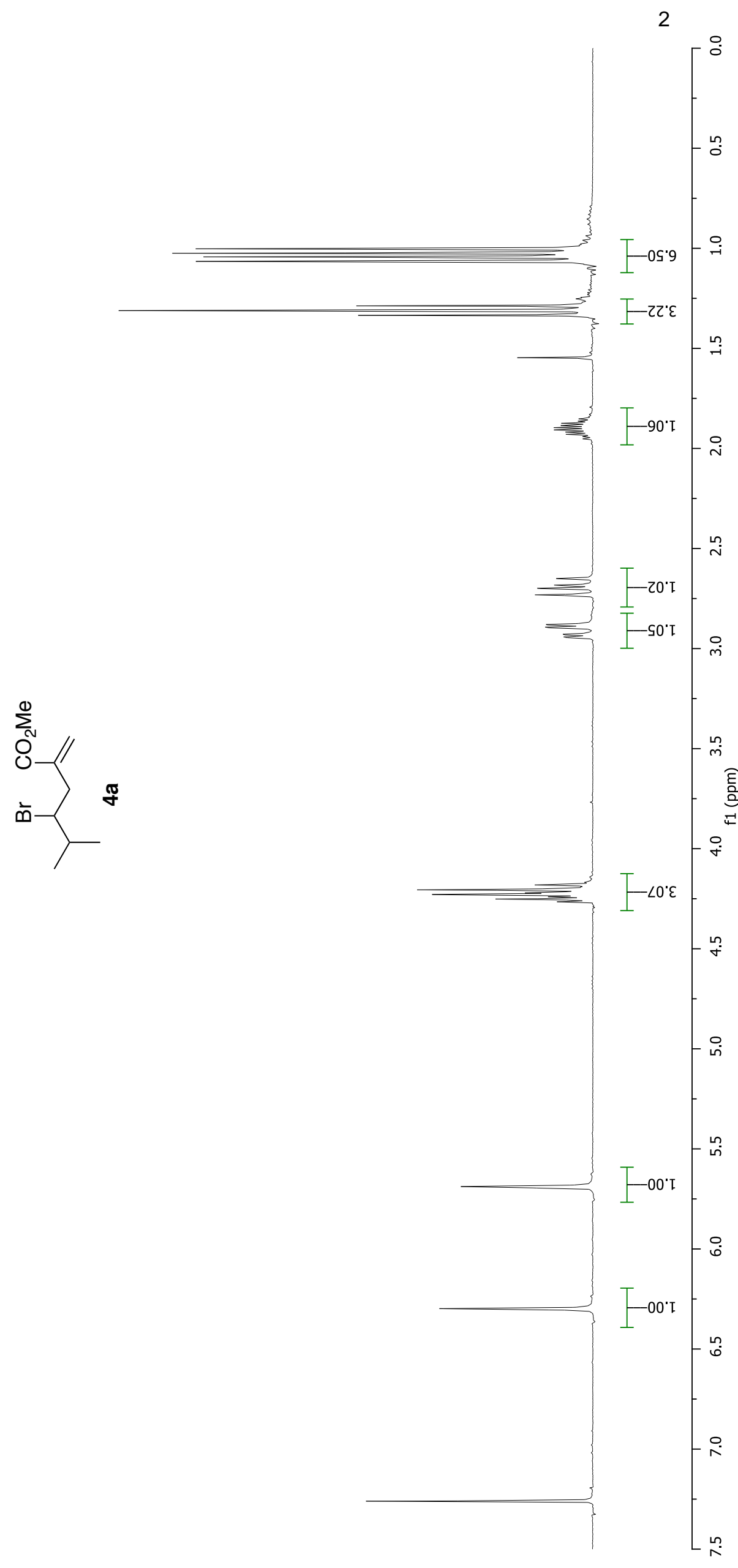


$\varepsilon^{\prime} \triangleright I-$

$0.8 \mathrm{I}-$

I'IZ -

$s^{\prime} \downarrow \varepsilon-$

$8.6 \varepsilon-$

0.โ9-

$8^{\circ} \varepsilon 9-$

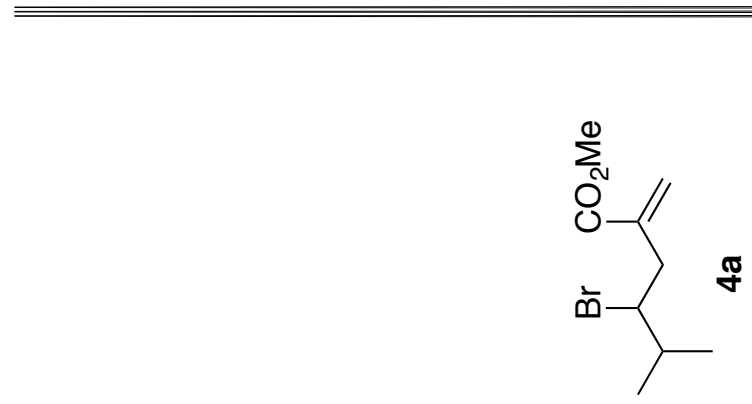

I. 82 I -

$9^{\circ} \angle \varepsilon I-$

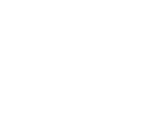

${ }^{0}$

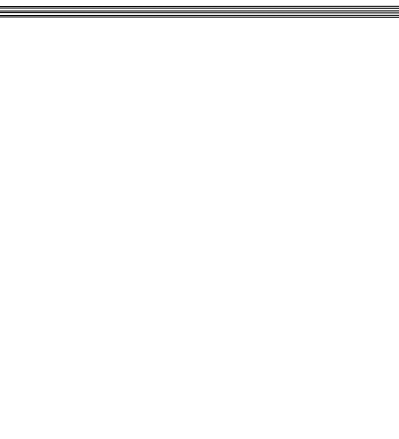

8.99I -

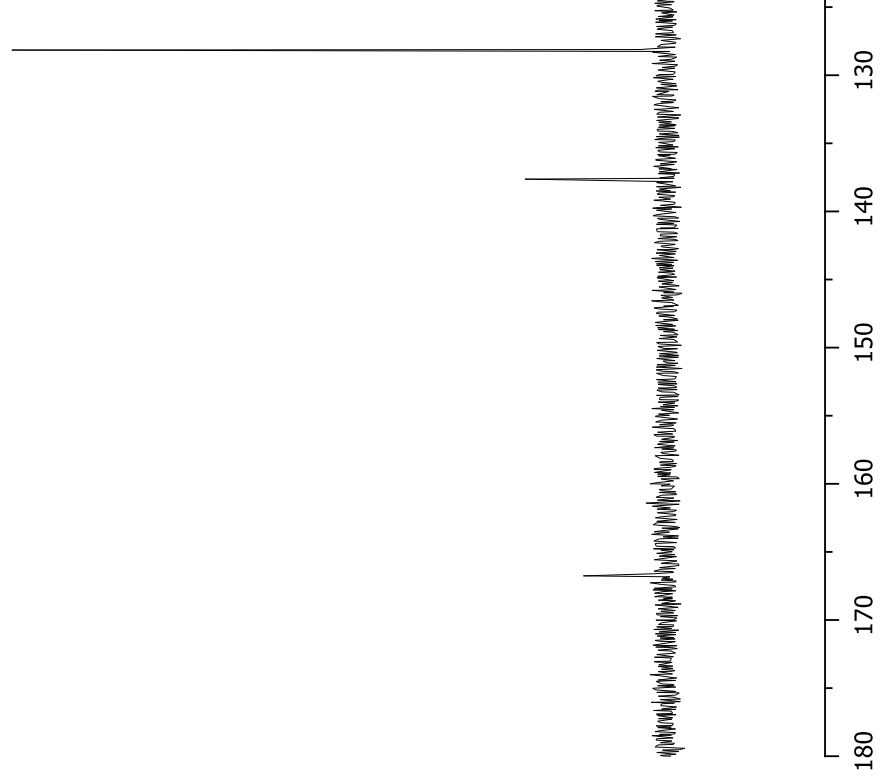




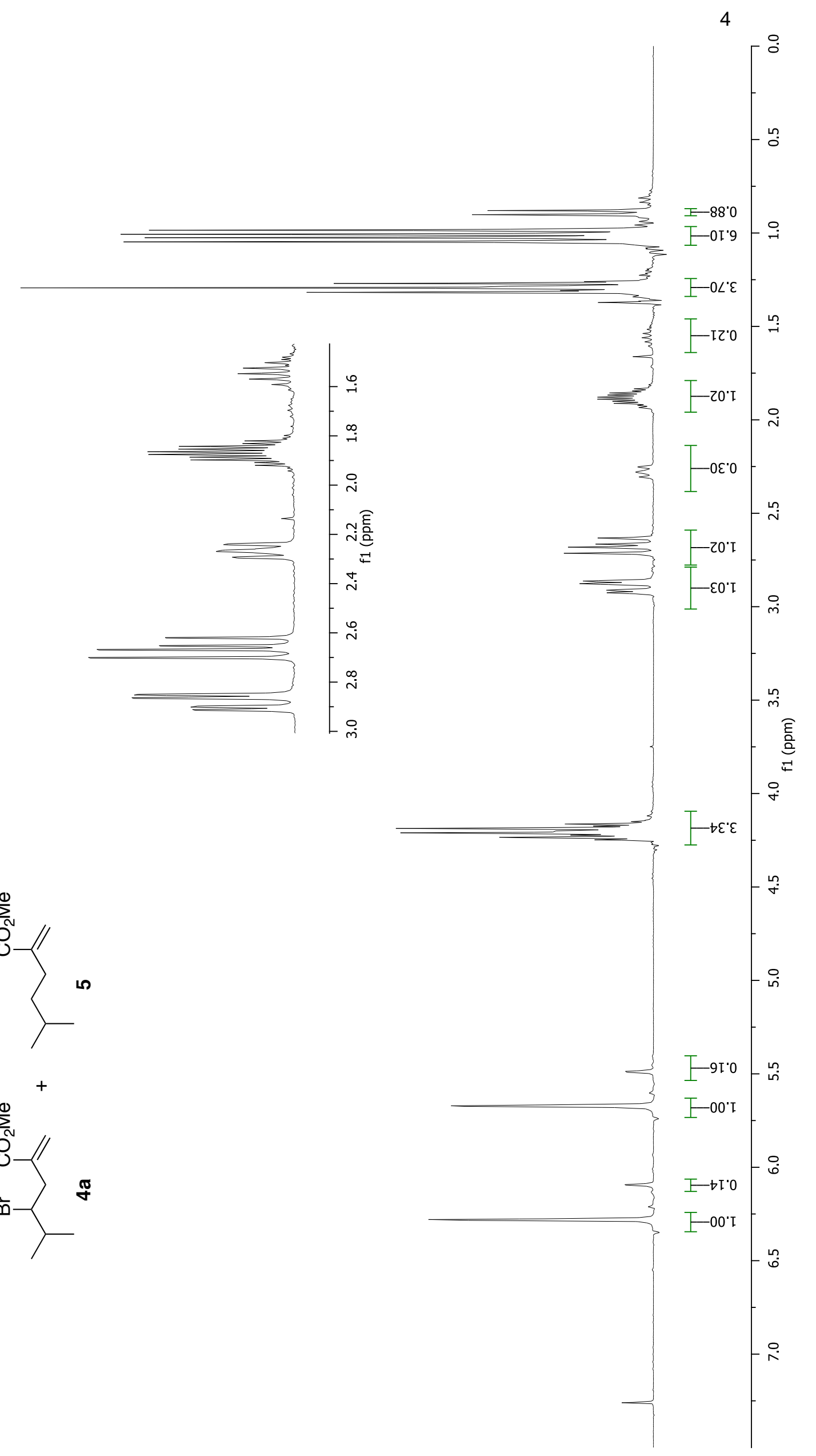


$6: \angle 2-$

$t^{\circ} t \varepsilon-$

$8 \angle \varepsilon-$

$\angle " 6 \varepsilon-$

9.09
6.09
2.89

L'E9 -

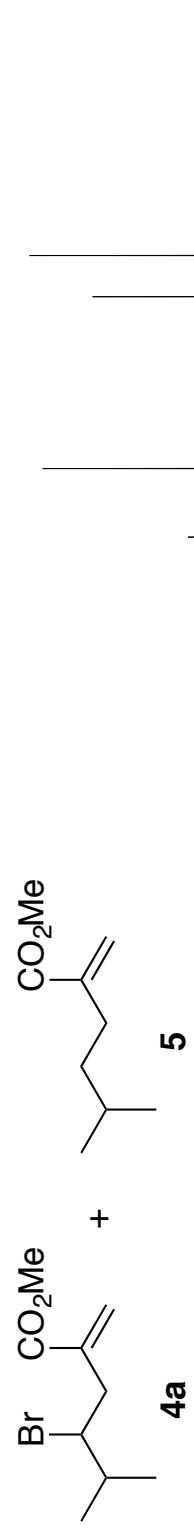

$0 \circ \mathrm{BLI}$

I. $82 \mathrm{I}$

$9^{\circ} \angle \varepsilon I-$

s.ItI -

$\angle 99 \mathrm{I}$

$\angle ' \angle 9 I-$

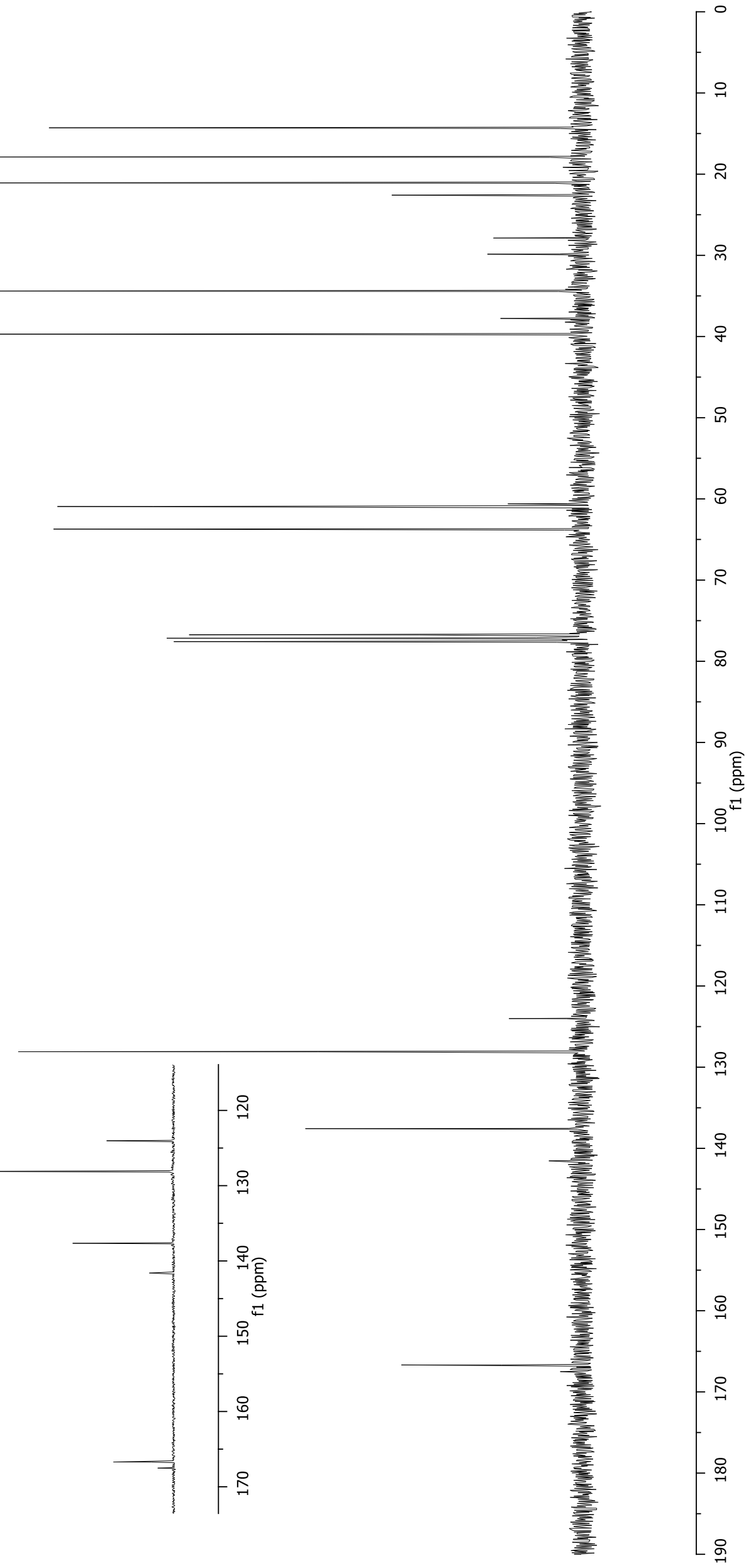




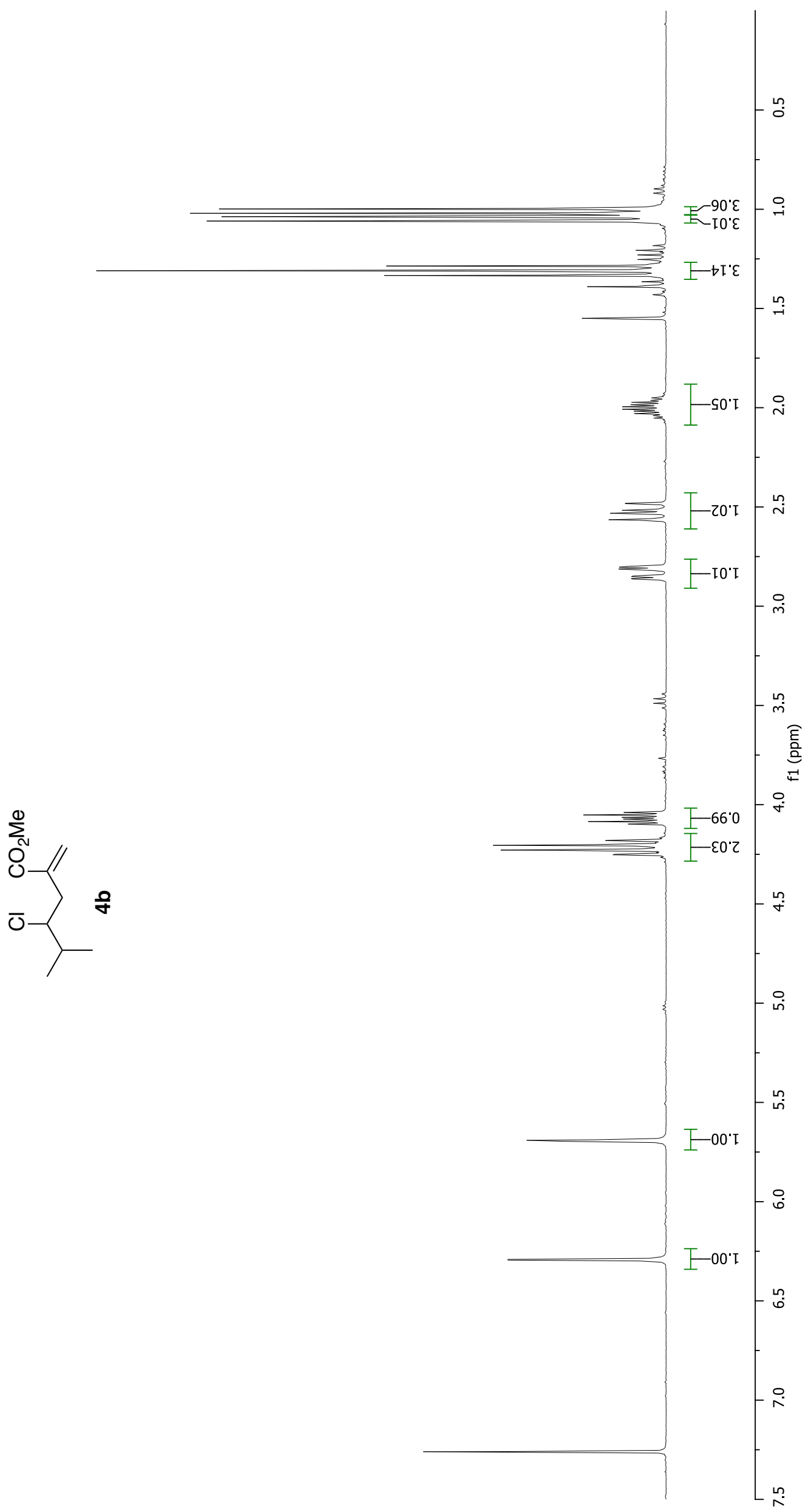




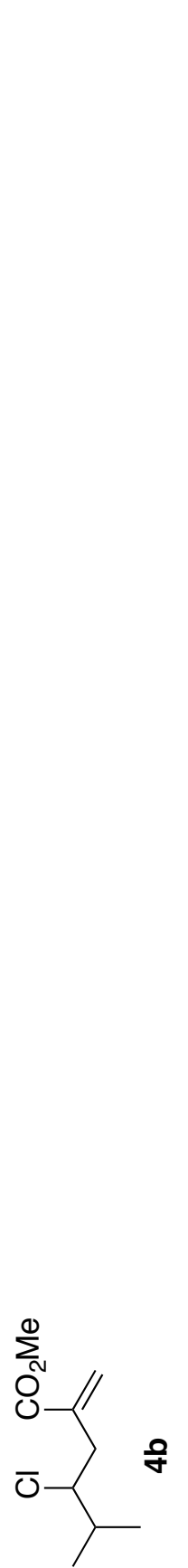

I.8ZI -

I'LEI -

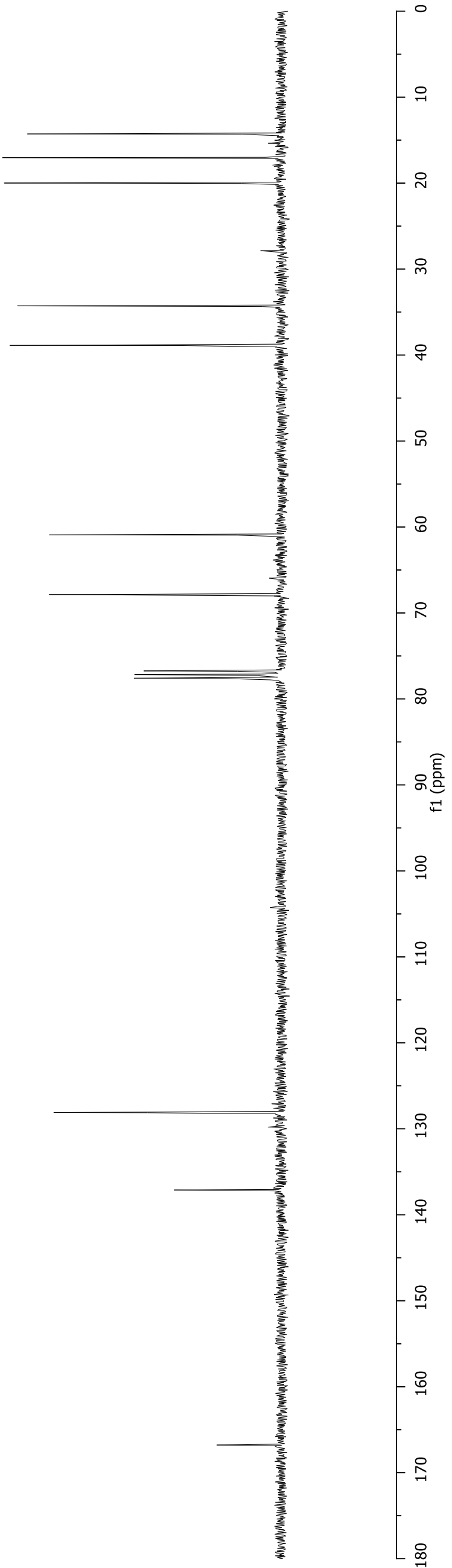




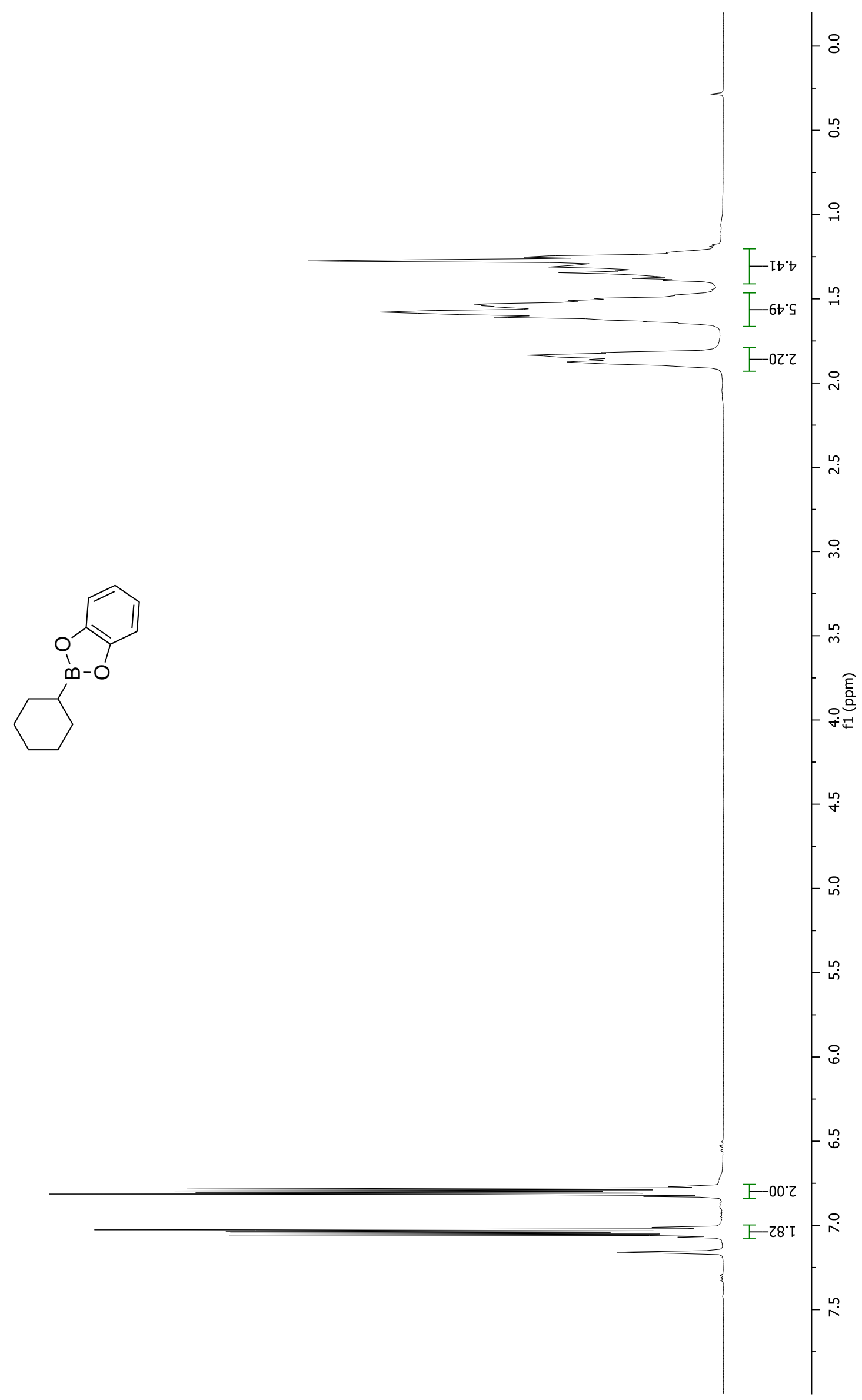


$\left.\begin{array}{l}0^{\prime} z z- \\ 0^{\prime}<z-\overline{-} \\ \varepsilon^{\prime}\langle z \\ z^{\prime} 8 z\end{array}\right]$

9'ZII -

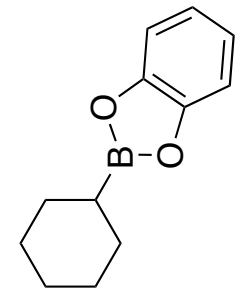

우

ํ.

요

q

in

8

ㅇ

$-\infty$

व $^{\text {틈 }}$

움

욱

్ำ

L'ZZI -

$68 \mathrm{DI}-$

욕

$\stackrel{\circ}{\circ}$

$\stackrel{7}{7}$ 


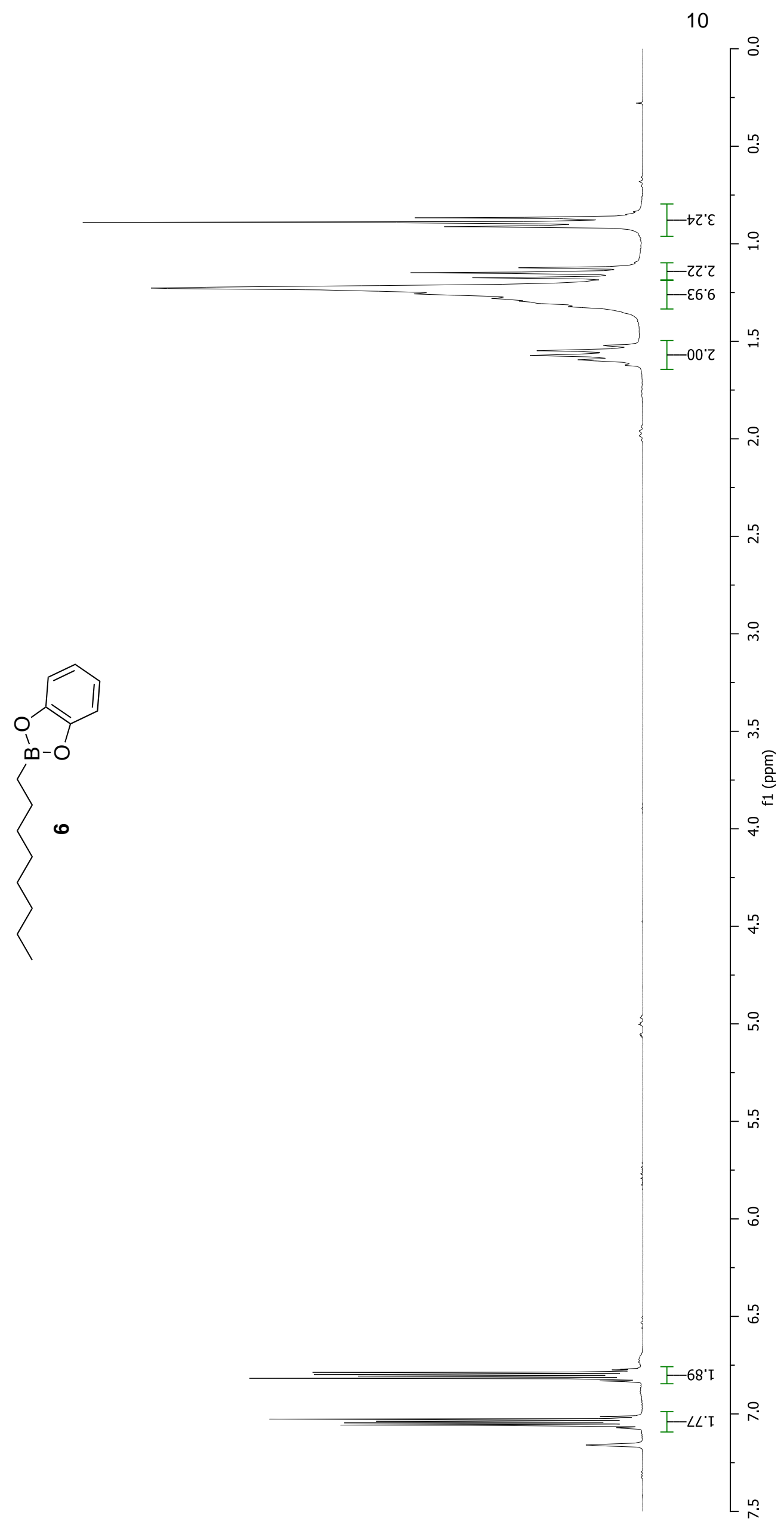


0.I -

เ๋เI-

โ'ยZ

โ'เ乙-

9.62-

8.62

$\varepsilon ' ح \varepsilon$

9'Zع

S'ZII -

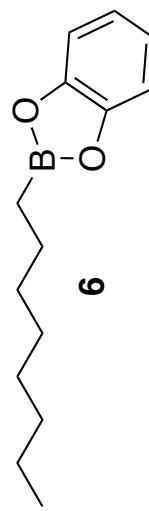

L'ZZI -

6.8tI -

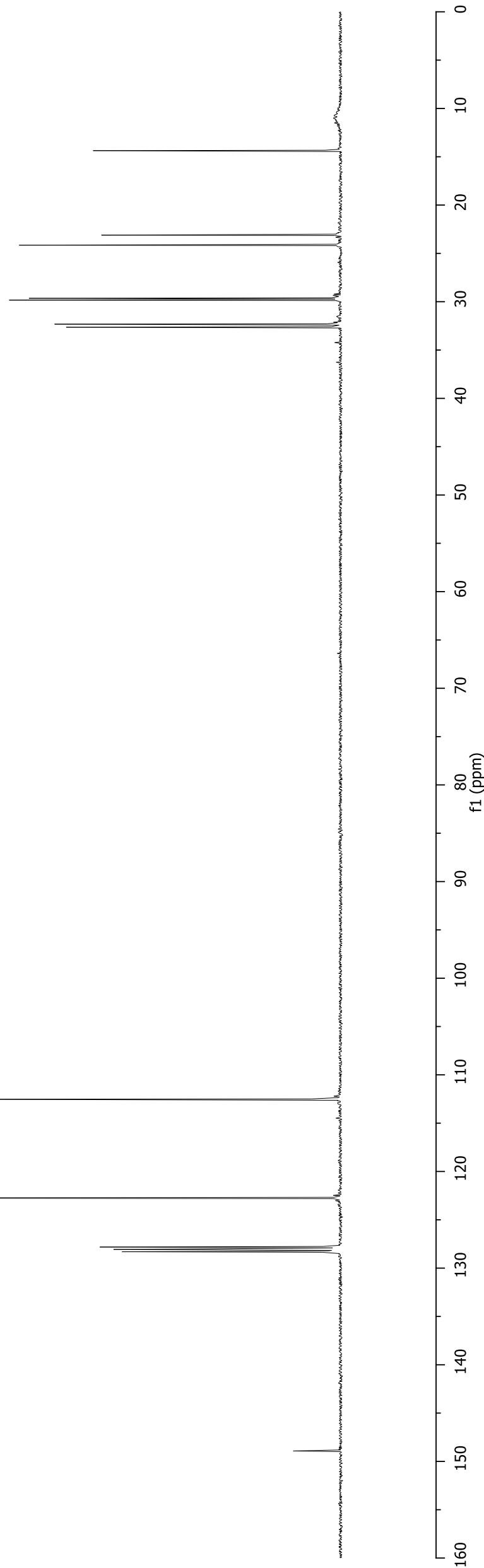




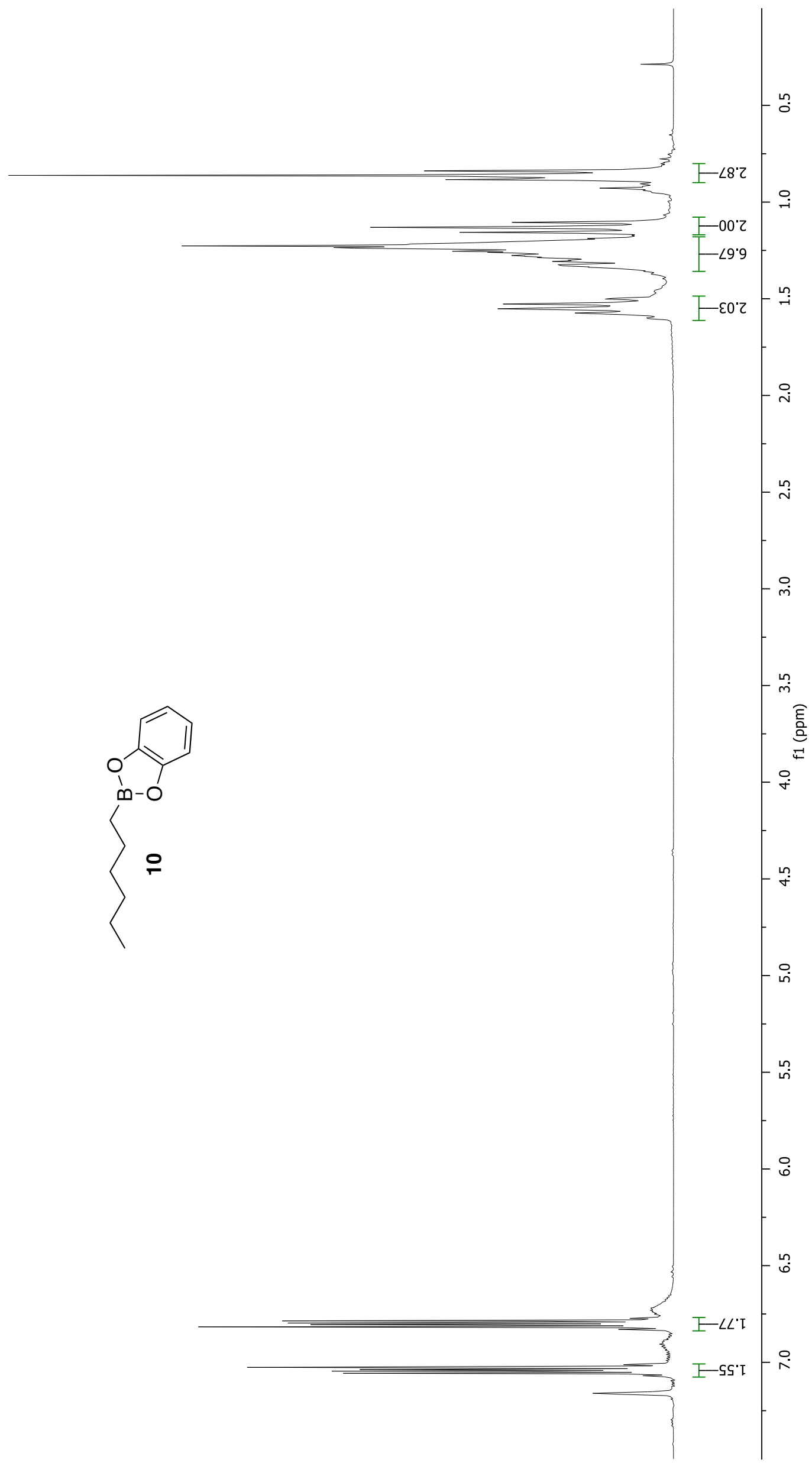


0.II-

$\varepsilon^{\prime \prime} \downarrow I-$

$0 . \varepsilon Z=$

I.

$\left.\begin{array}{l}0{ }^{\circ} z \varepsilon \\ \varepsilon^{\prime} z \varepsilon\end{array}\right\}$

S'ZII -

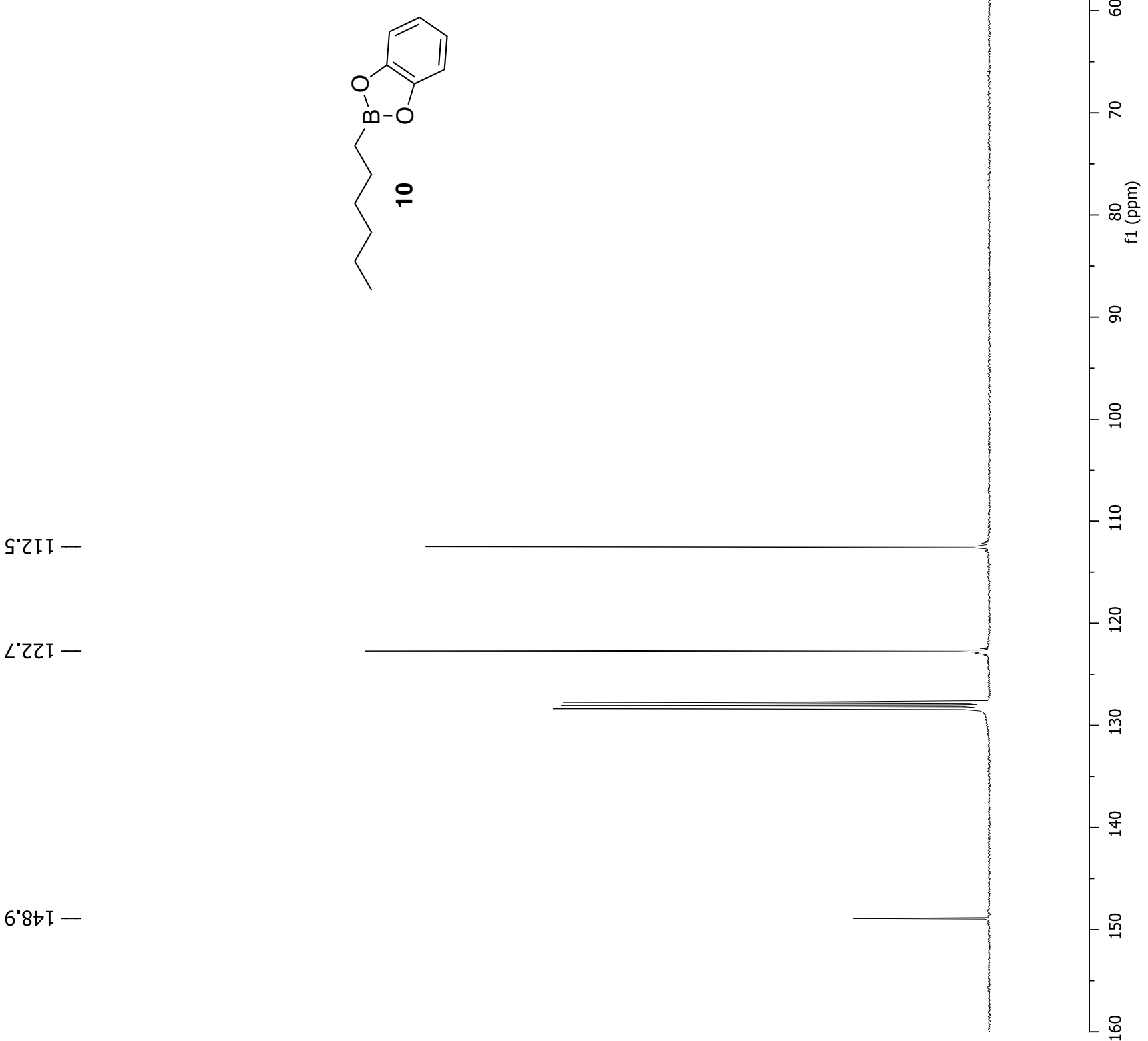




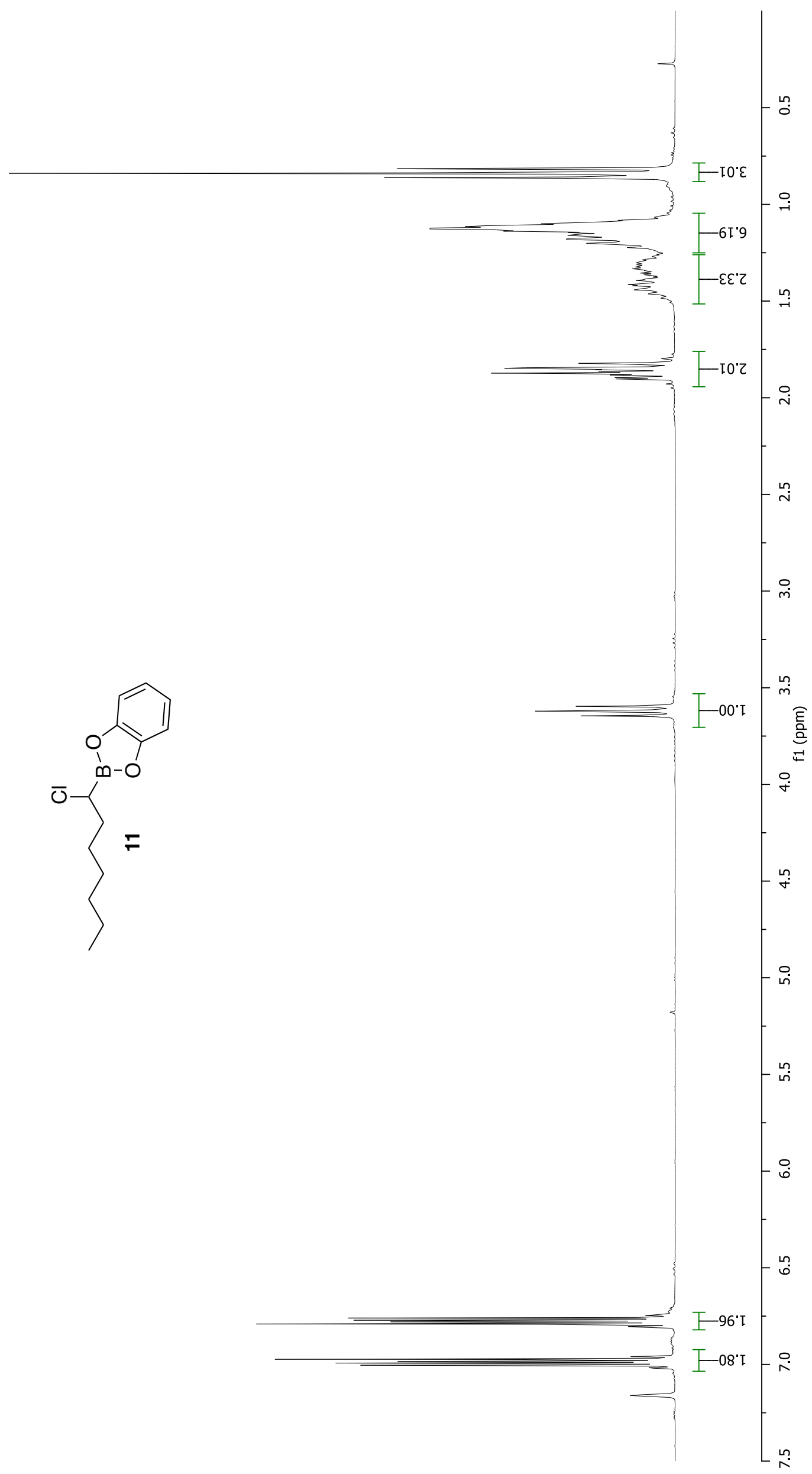


$\varepsilon^{*} \triangleright I$

$6 \cdot 22-$

$s^{\circ} \angle Z-$

6. $1 \varepsilon-$

$b^{\circ} \downarrow \varepsilon-$

でてー

0.EI -
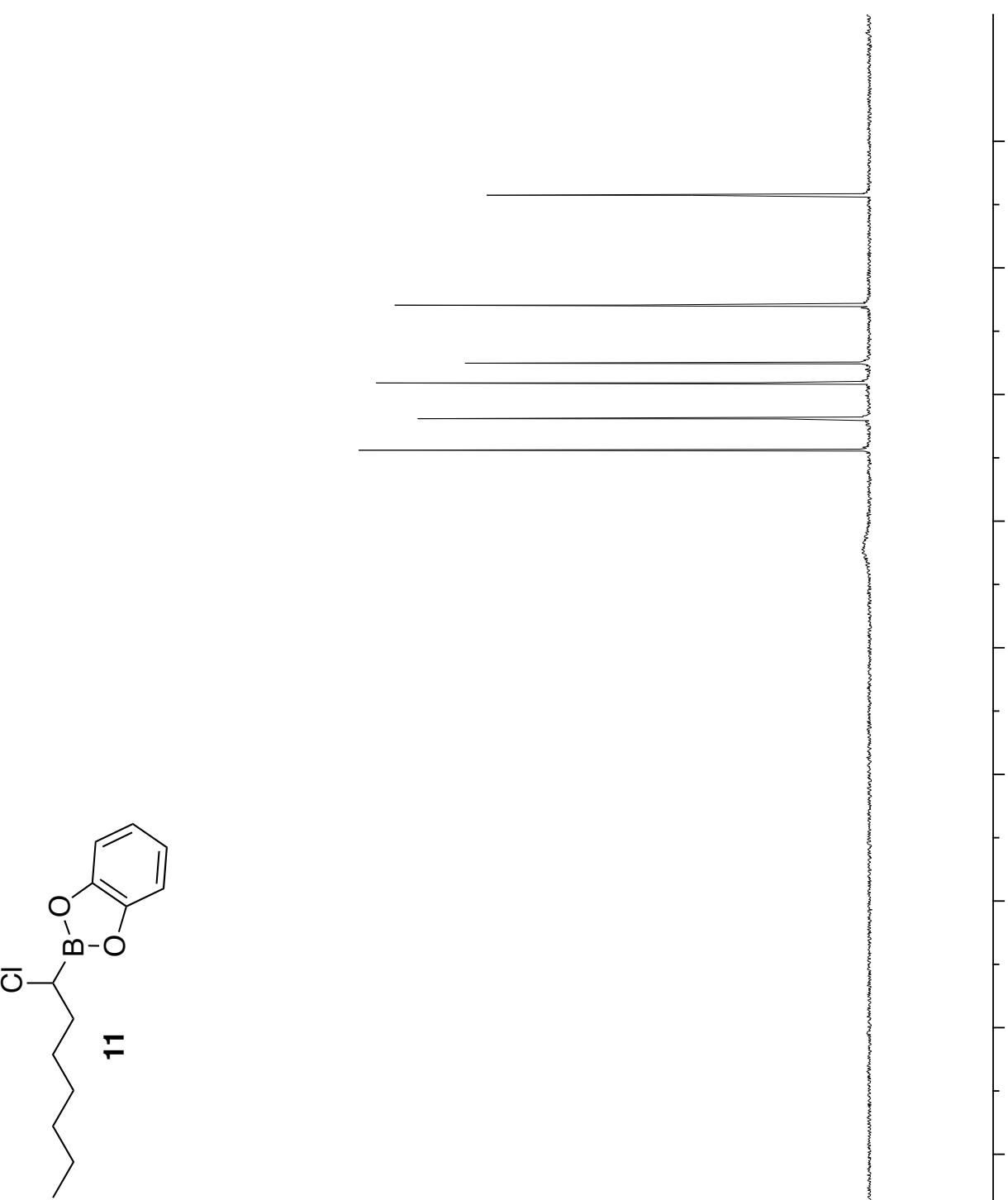

๑๋ยZI -

t.8tI -

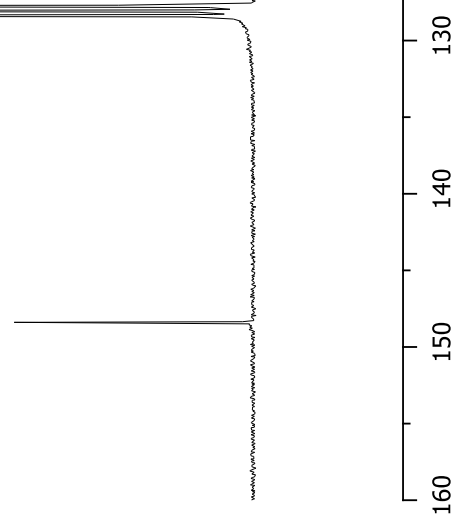




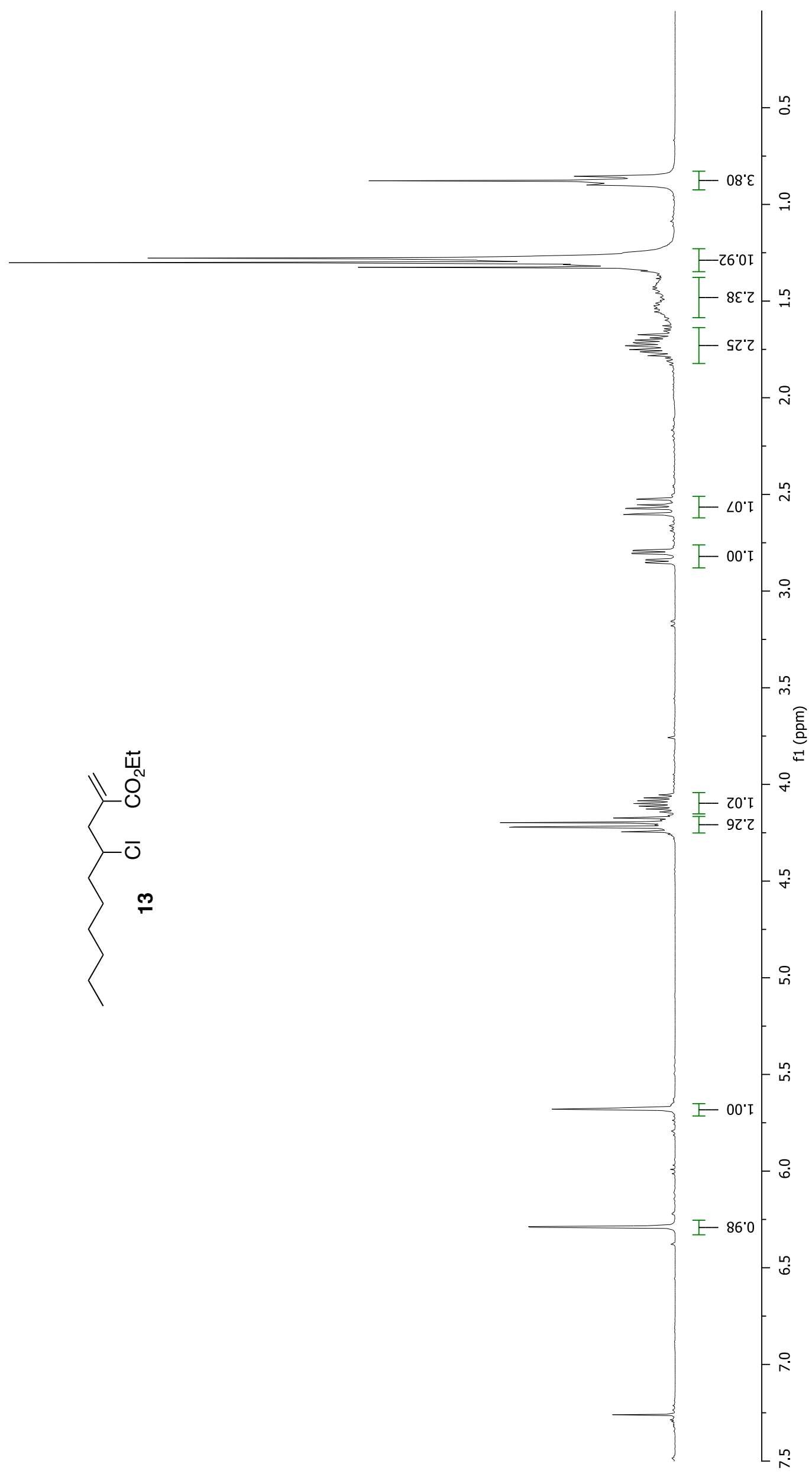


$Z^{*} \downarrow I$
$\varepsilon \neq I$

L'ZZ-

s.92 -

8. $T \varepsilon-$

$5^{\circ} 8 \varepsilon-$

9. I

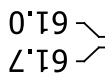
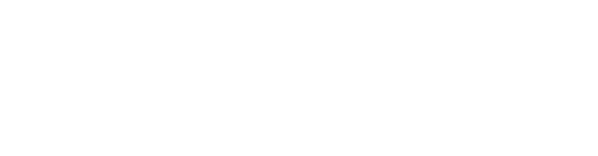

${ }^{0}$

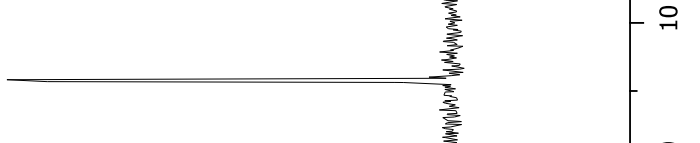

$-\frac{1}{2}$

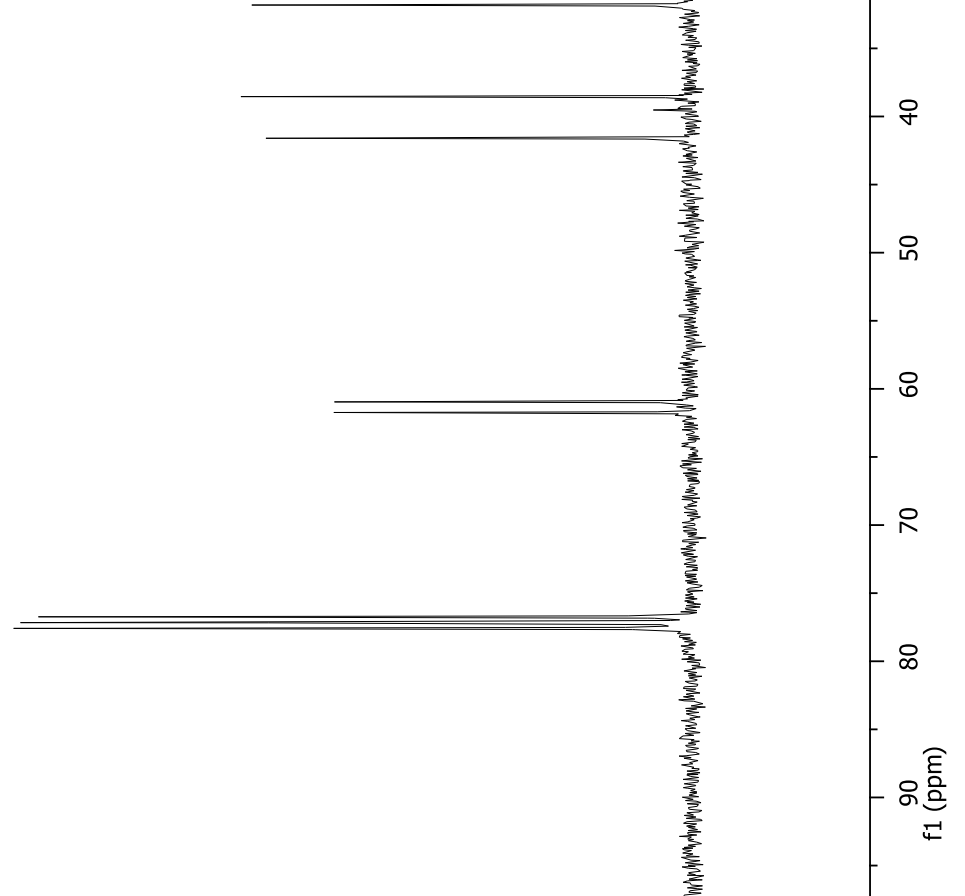

$\sum\langle\overline{0}$

$0^{\circ} \angle \varepsilon I-$

8.99I -

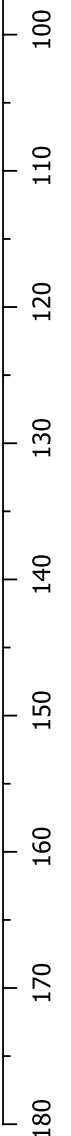




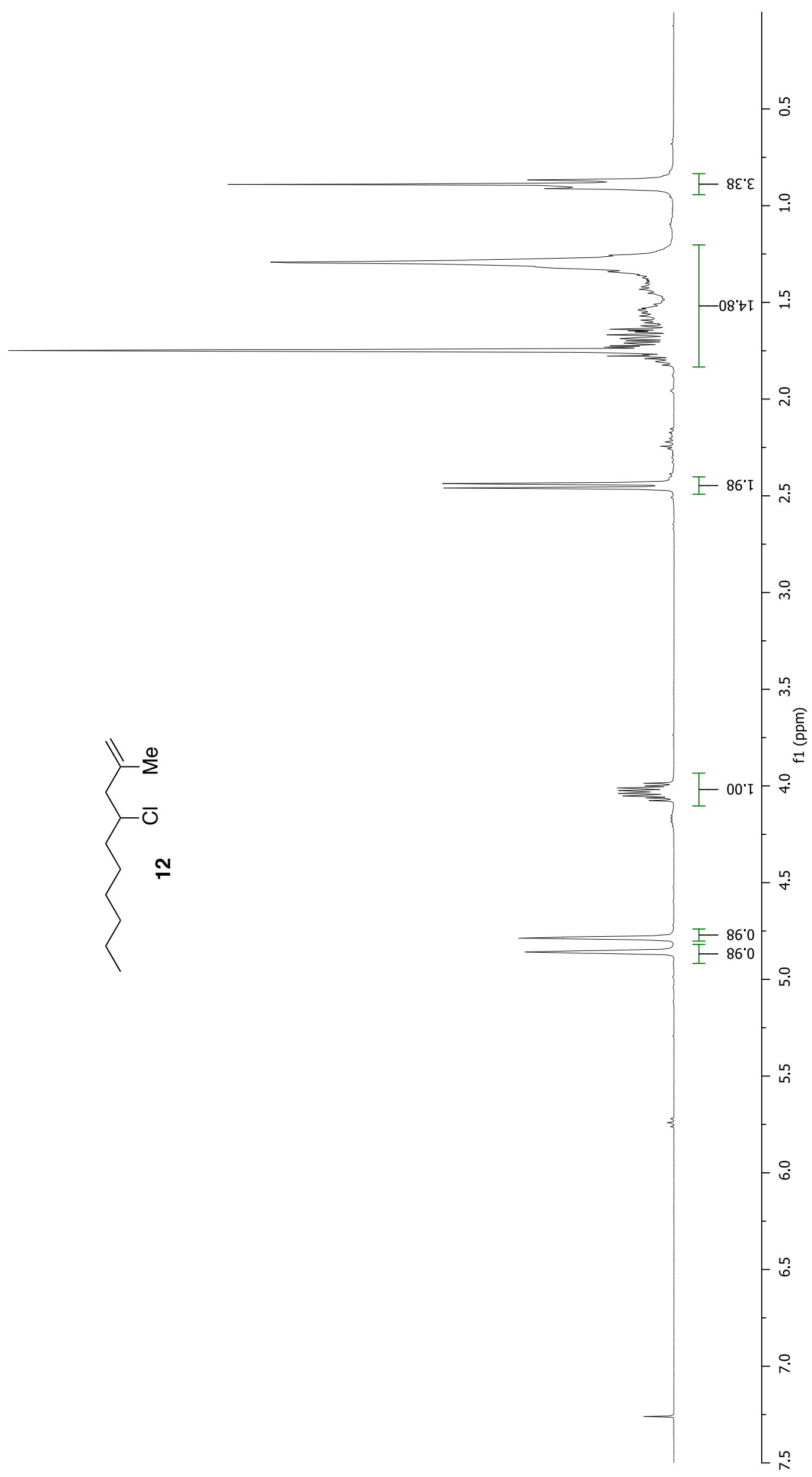


$Z^{\circ} \downarrow I$

$\varepsilon: 22$

s.92 -

6. $1 \varepsilon-$

$\varepsilon^{\prime} 8 \varepsilon-$

$\tau^{\prime} \angle t-$

ย'โ9-

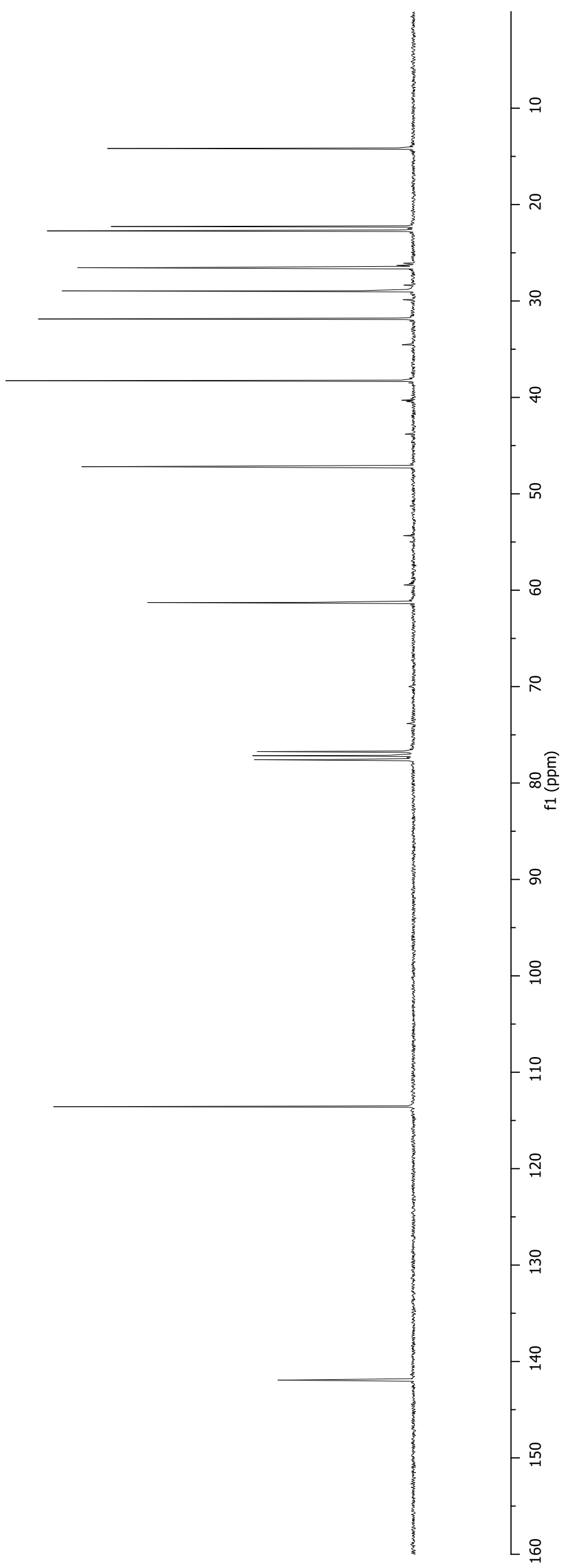

6.ItI -

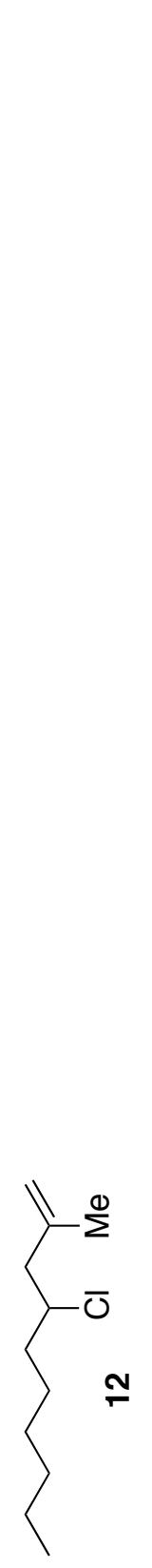

9'EI -

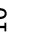




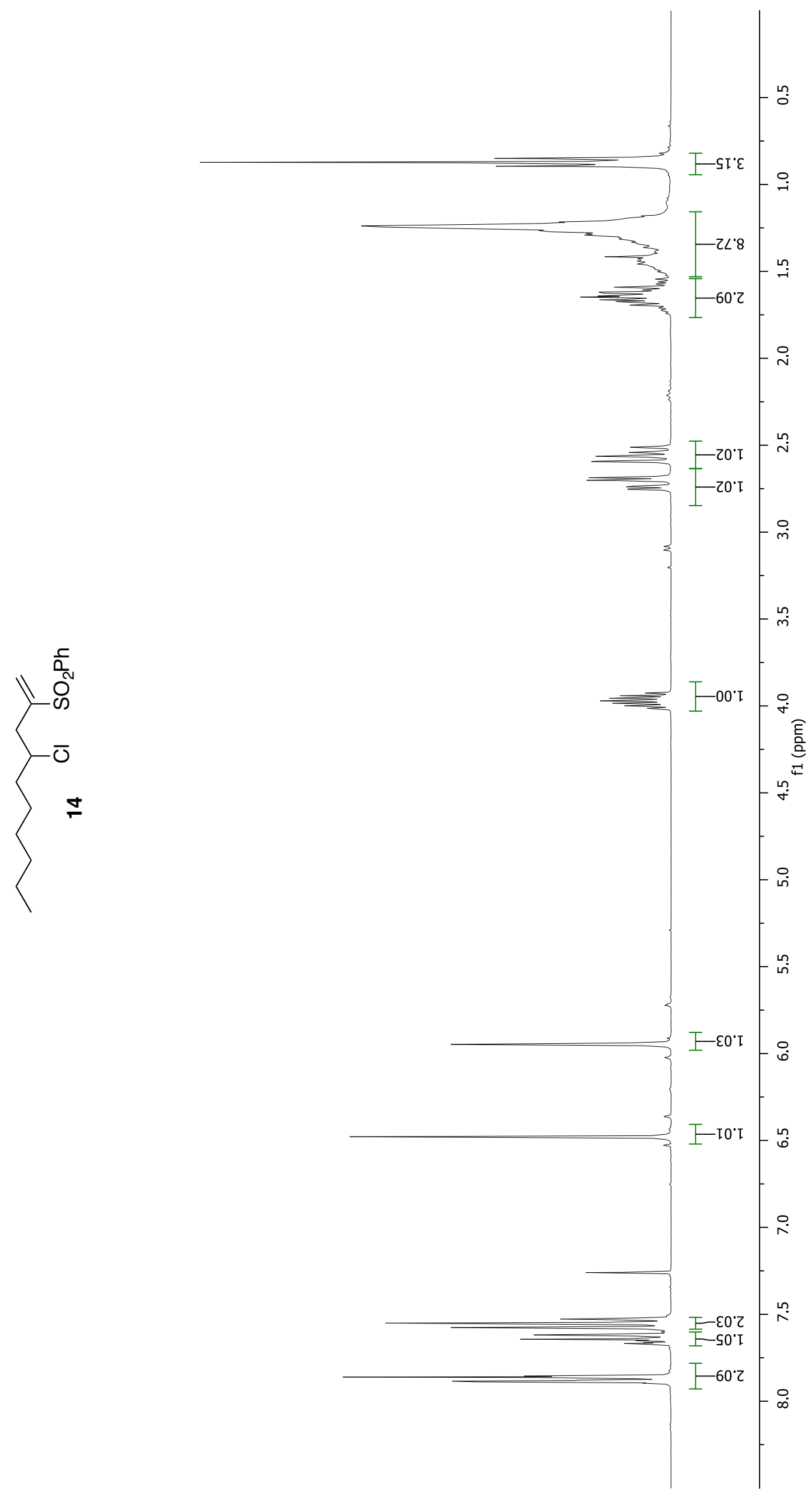


I" $\triangleright I$

$9.22-$

$\varepsilon \cdot 92-$

L'IE-

$\varepsilon^{\circ} 8 \varepsilon$

I'6ع

I.09-

乙 $\angle$ ZI

๑8ZI ᄂ

$5^{\circ} 62 \mathrm{I}-$

$8^{\circ} \varepsilon \varepsilon I-$

8.8عI -

$9.9 b I-$

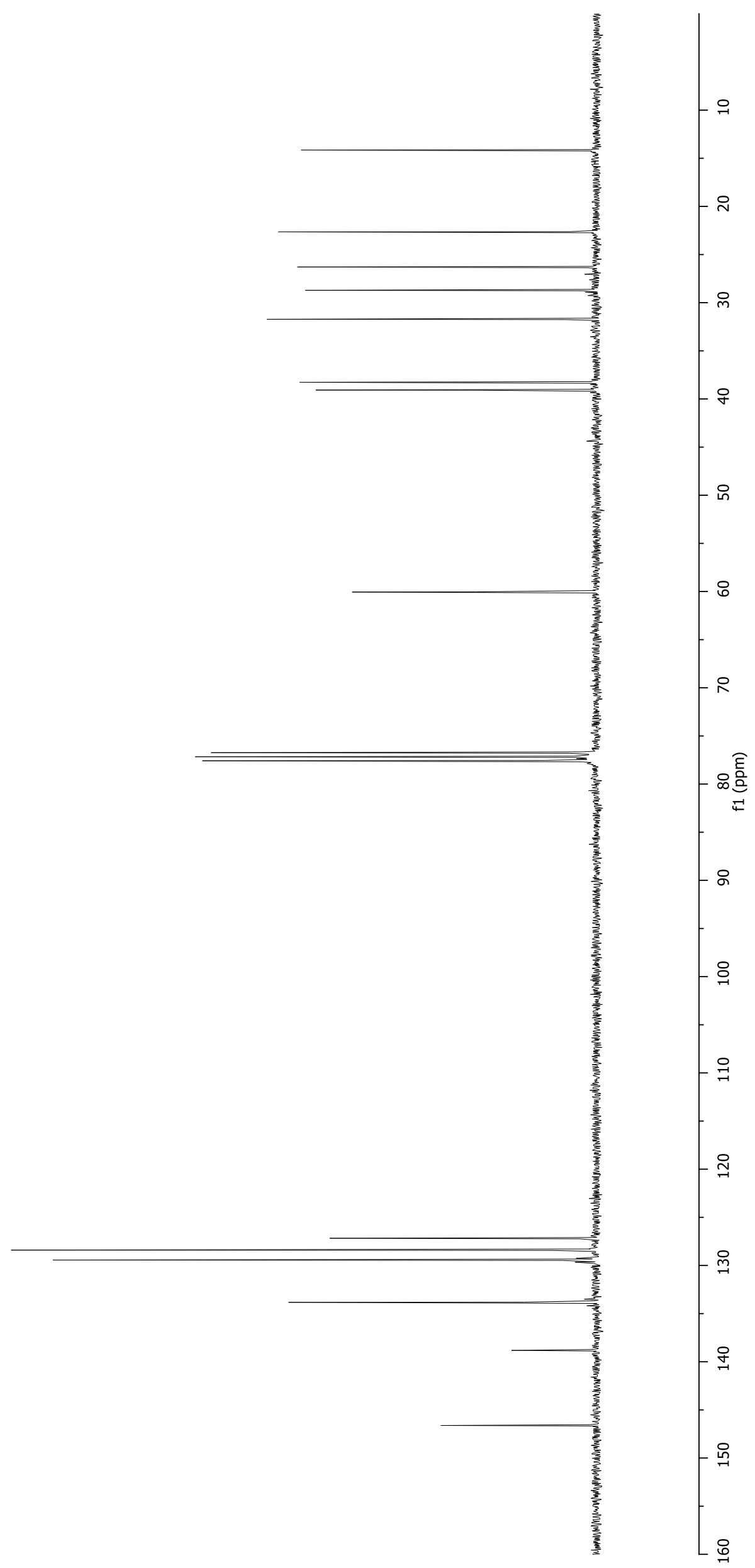




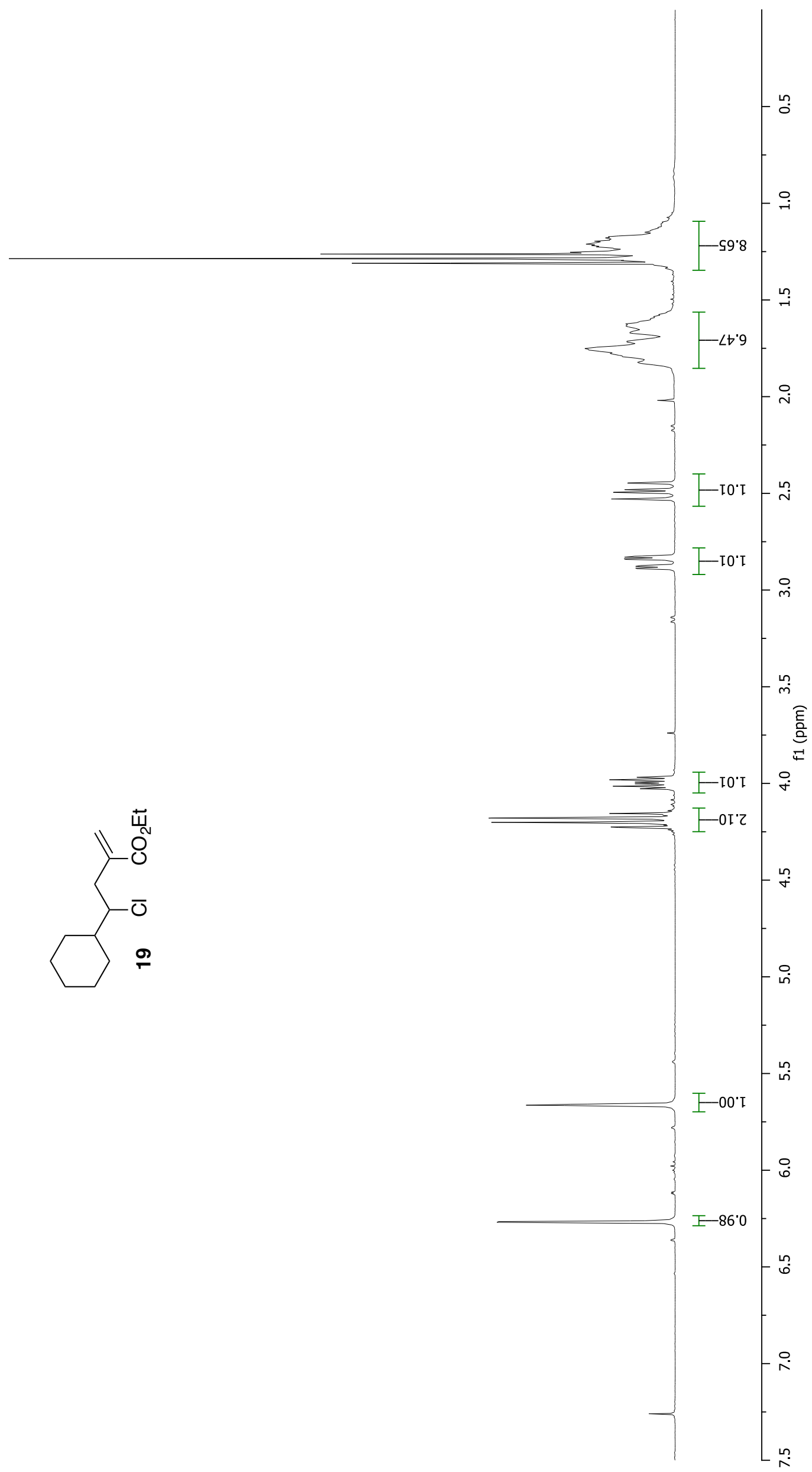


$\varepsilon^{*} \downarrow I-$

I'92

$\varepsilon " 92$

七.92

ح.0

$98 \varepsilon$

$\varepsilon " t \downarrow-$

6.09

I' $\angle 9-$

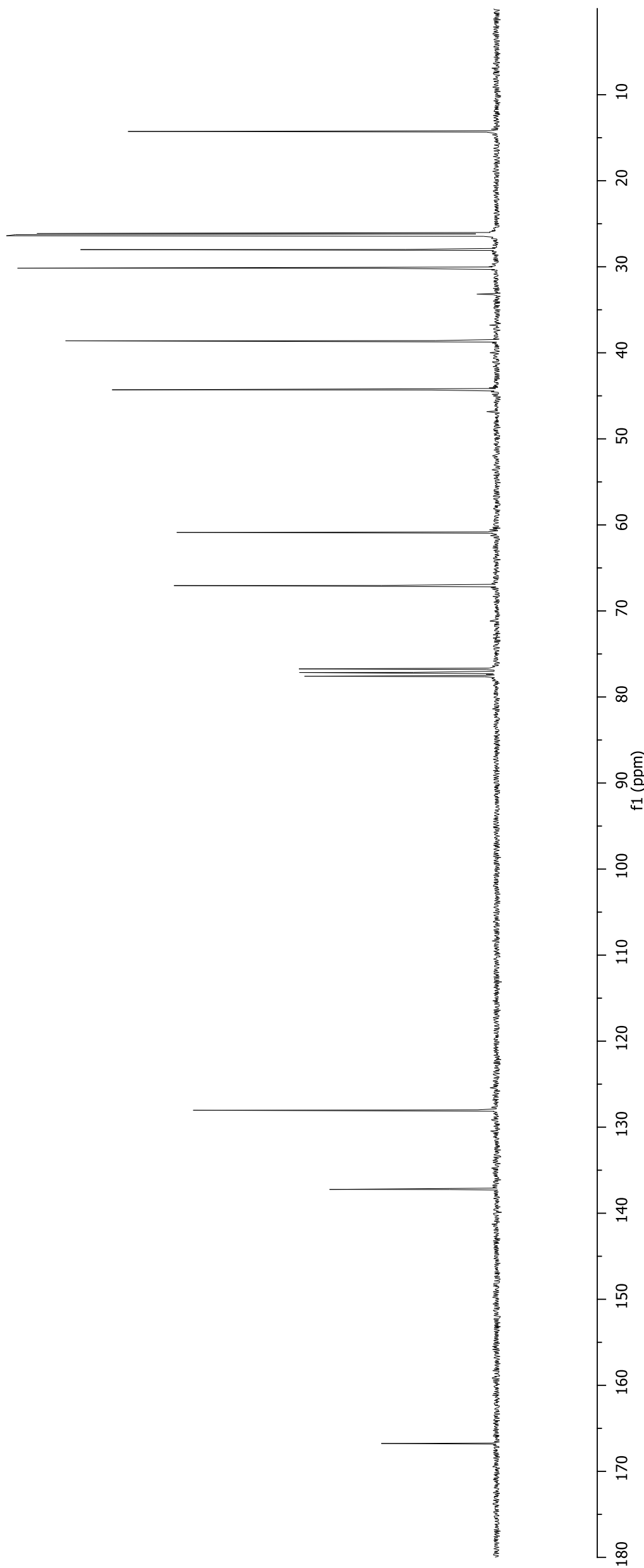

8.99I -

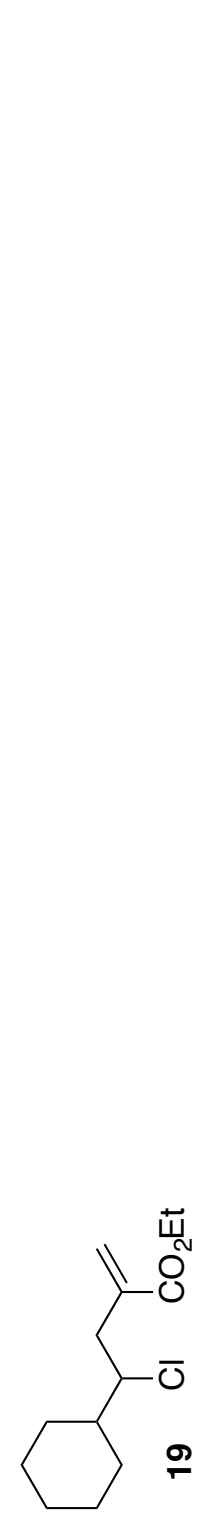

$0.82 \mathrm{I}$

$Z^{\prime} \angle \varepsilon I-$ 


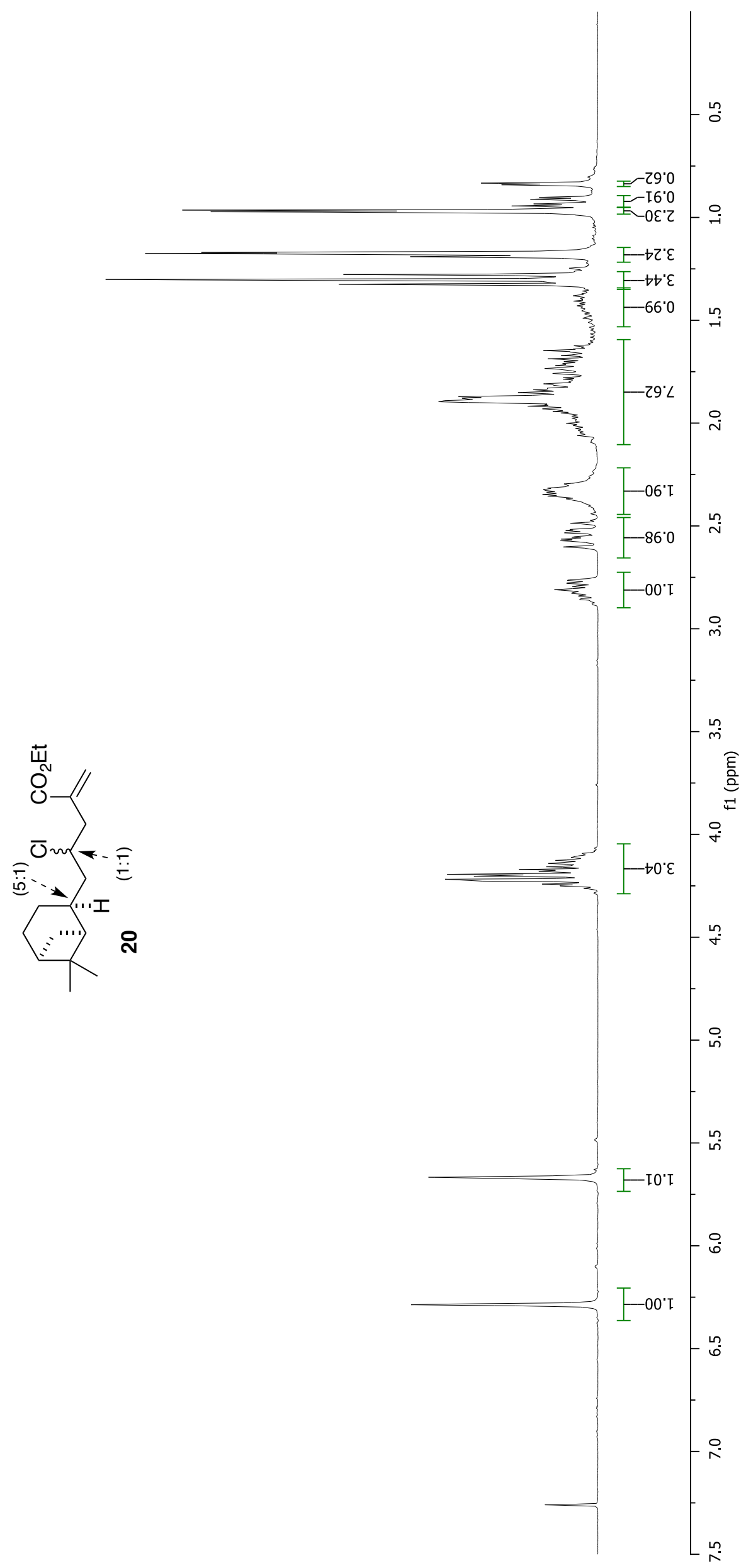



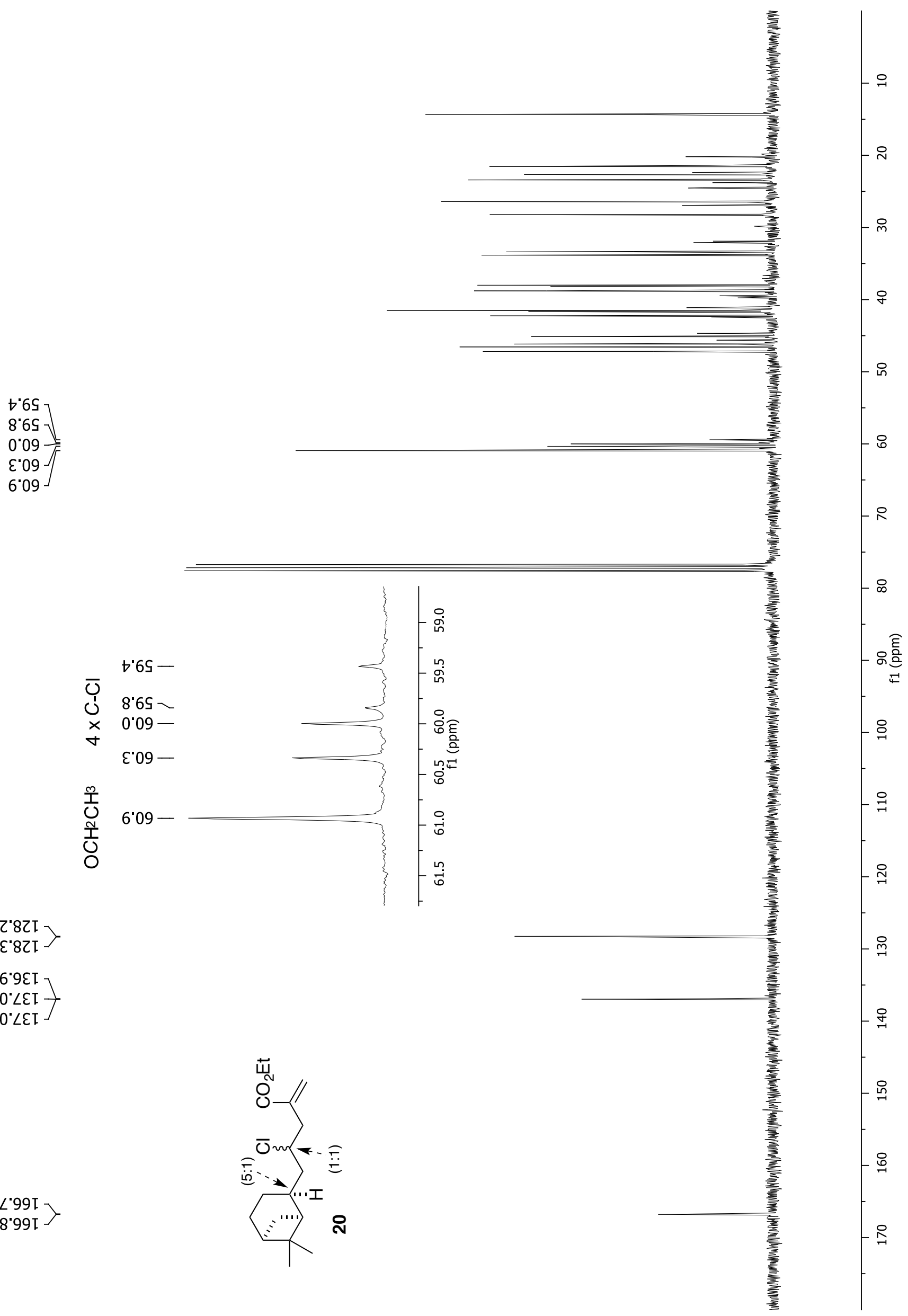

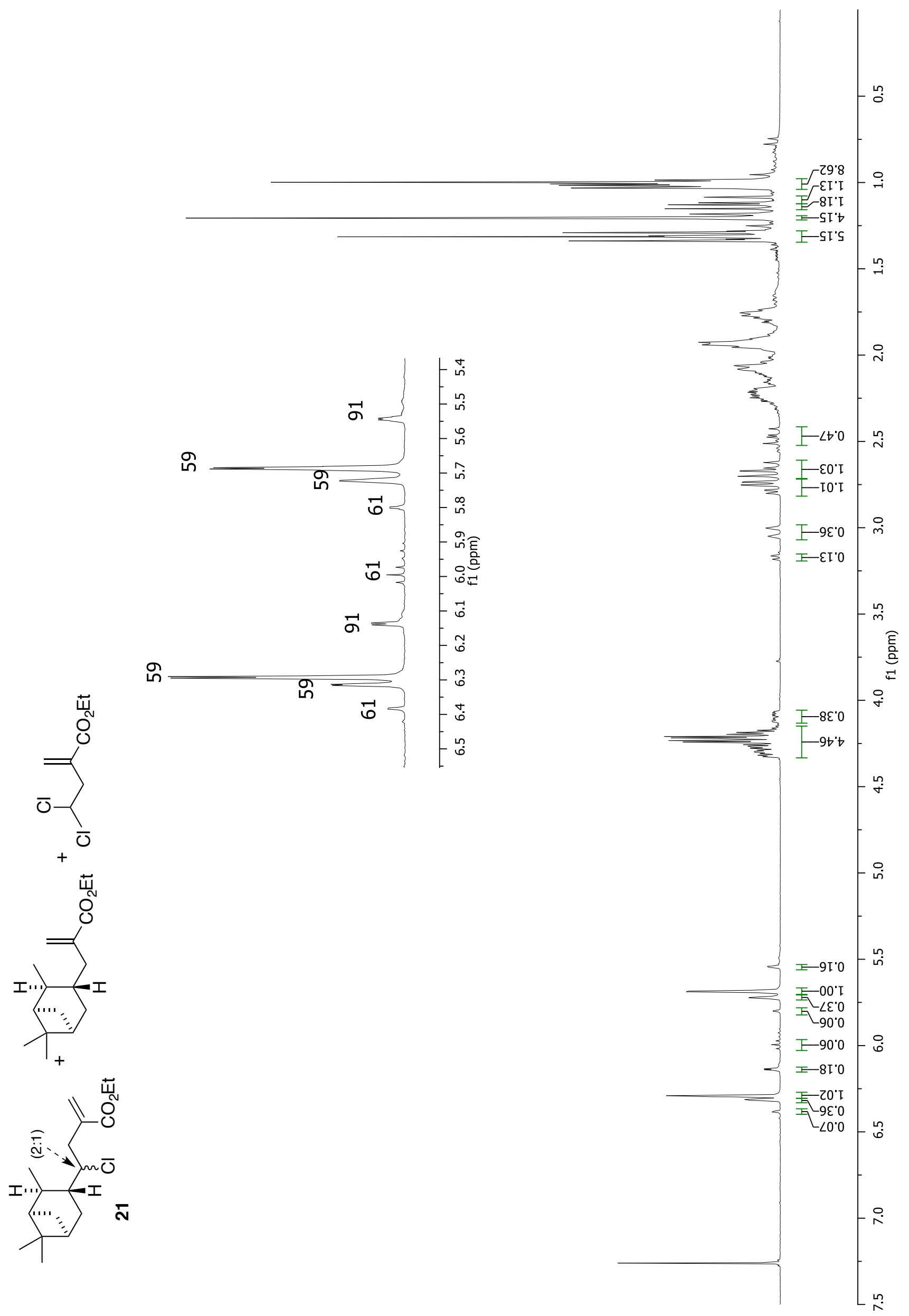


$$
\begin{aligned}
& \begin{array}{l}
\varepsilon * t I \\
\forall * t I
\end{array} \\
& \begin{array}{l}
b^{\prime} I Z \\
0 . \varepsilon Z \text { ᄂ }
\end{array} \\
& 8^{\circ} \angle Z- \\
& \text { †. IE- } \\
& \text { द:वह } \\
& \text { ح'6E- } \\
& \text { E'It- } \\
& \begin{array}{l}
9 \cdot \angle t \bar{~} \\
t^{\circ} 8 t
\end{array} \\
& \text { 0.โ9 } 0 \text {-โ9 } \\
& 6.99- \\
& 5.89^{-}
\end{aligned}
$$

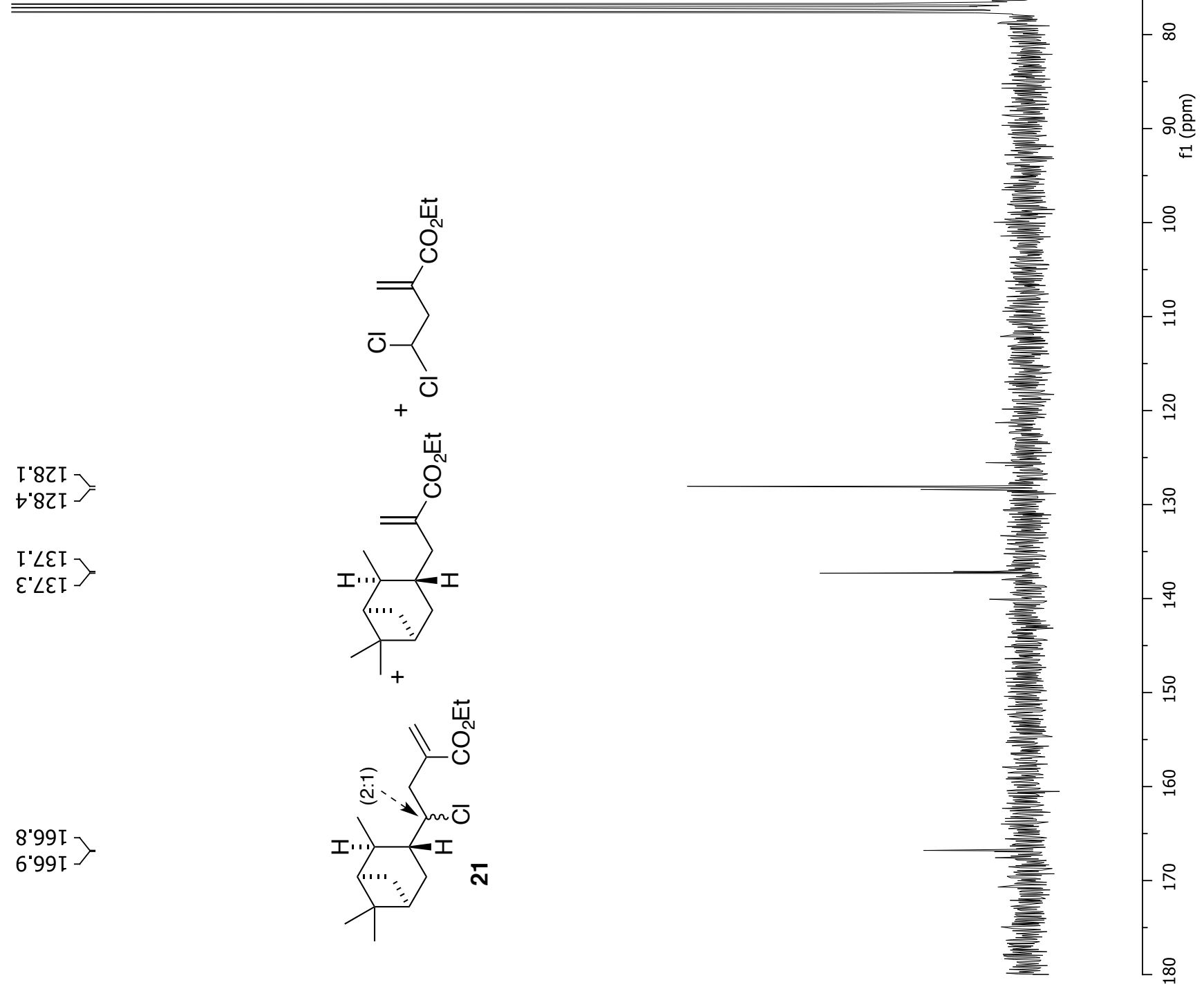




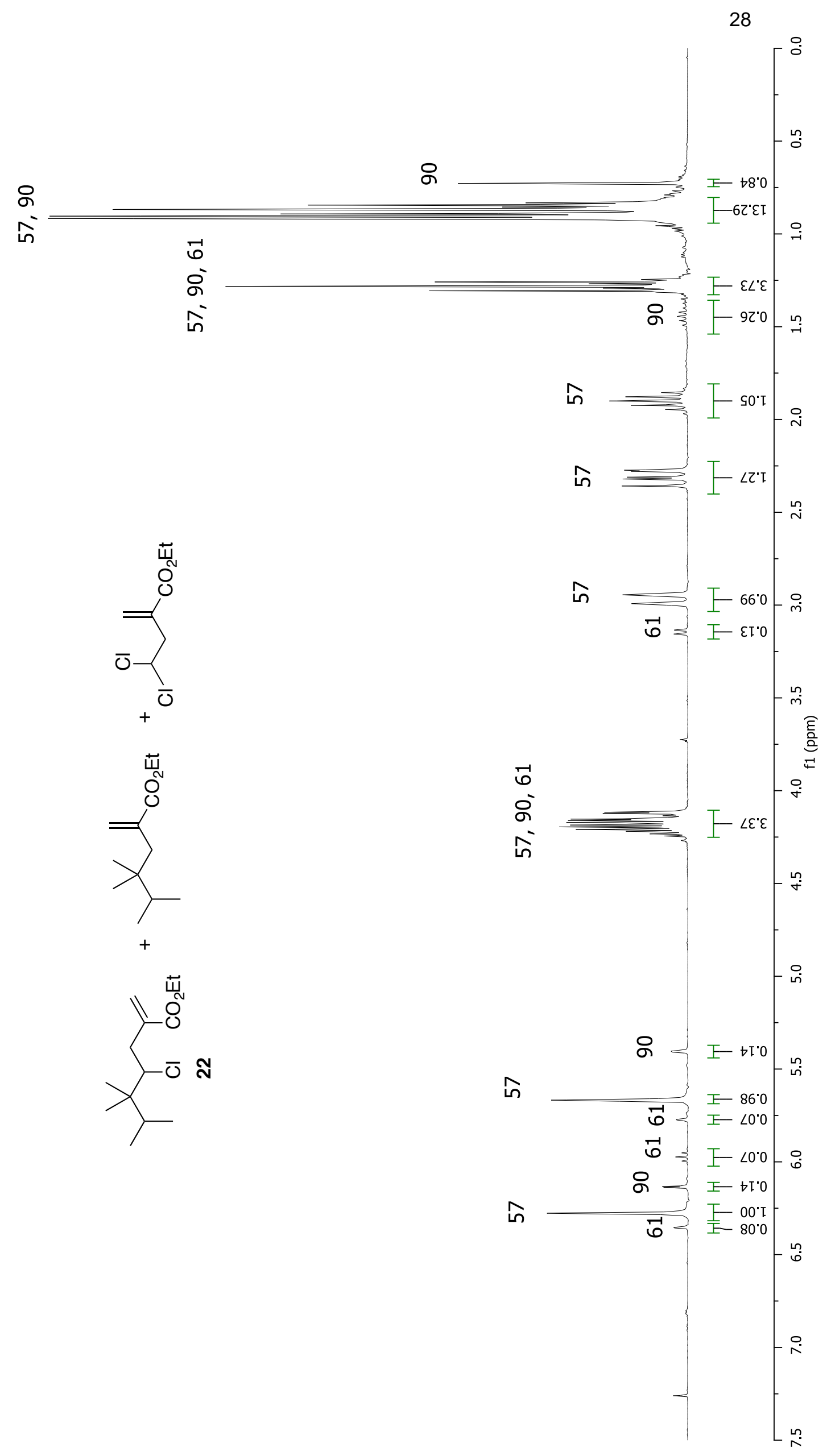




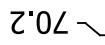

Z'IL

8.92I

$\checkmark 82 \mathrm{I}$

${ }^{\circ} 0 \varepsilon \mathrm{I}-$
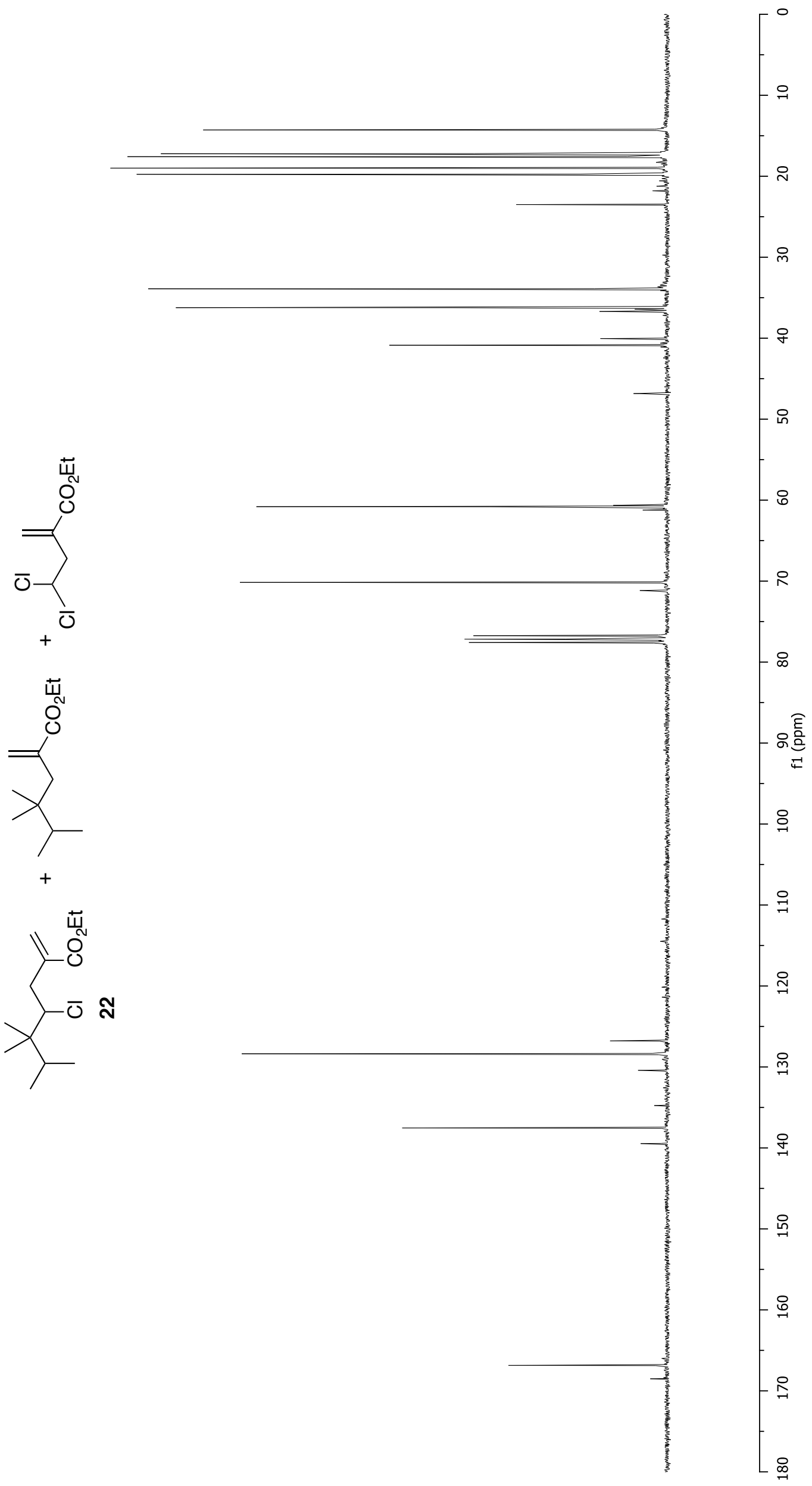

$8^{\circ} \triangleright \varepsilon I-$

S'LEI -

S'6ع I
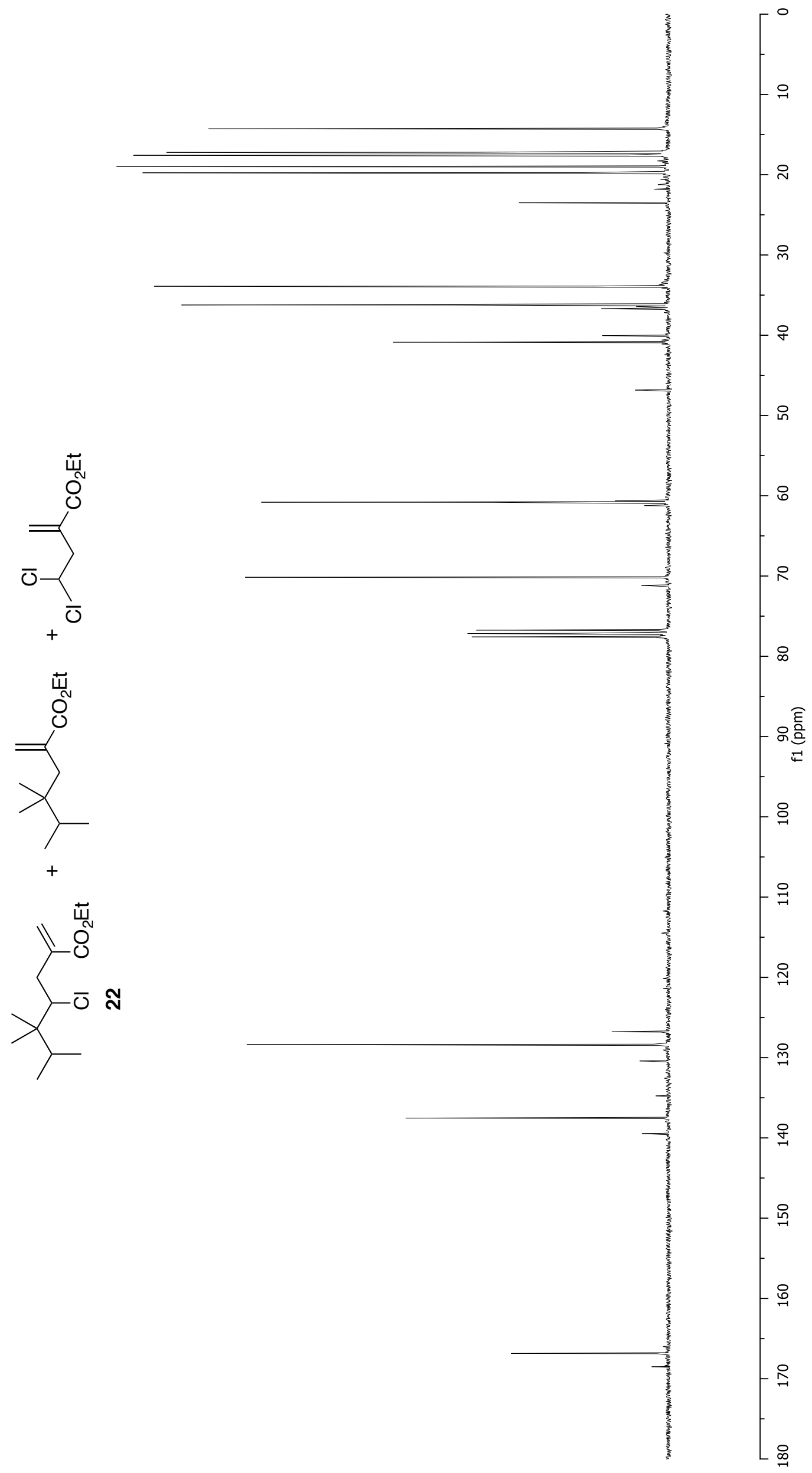

0.99I

6.99I -

S.89I 


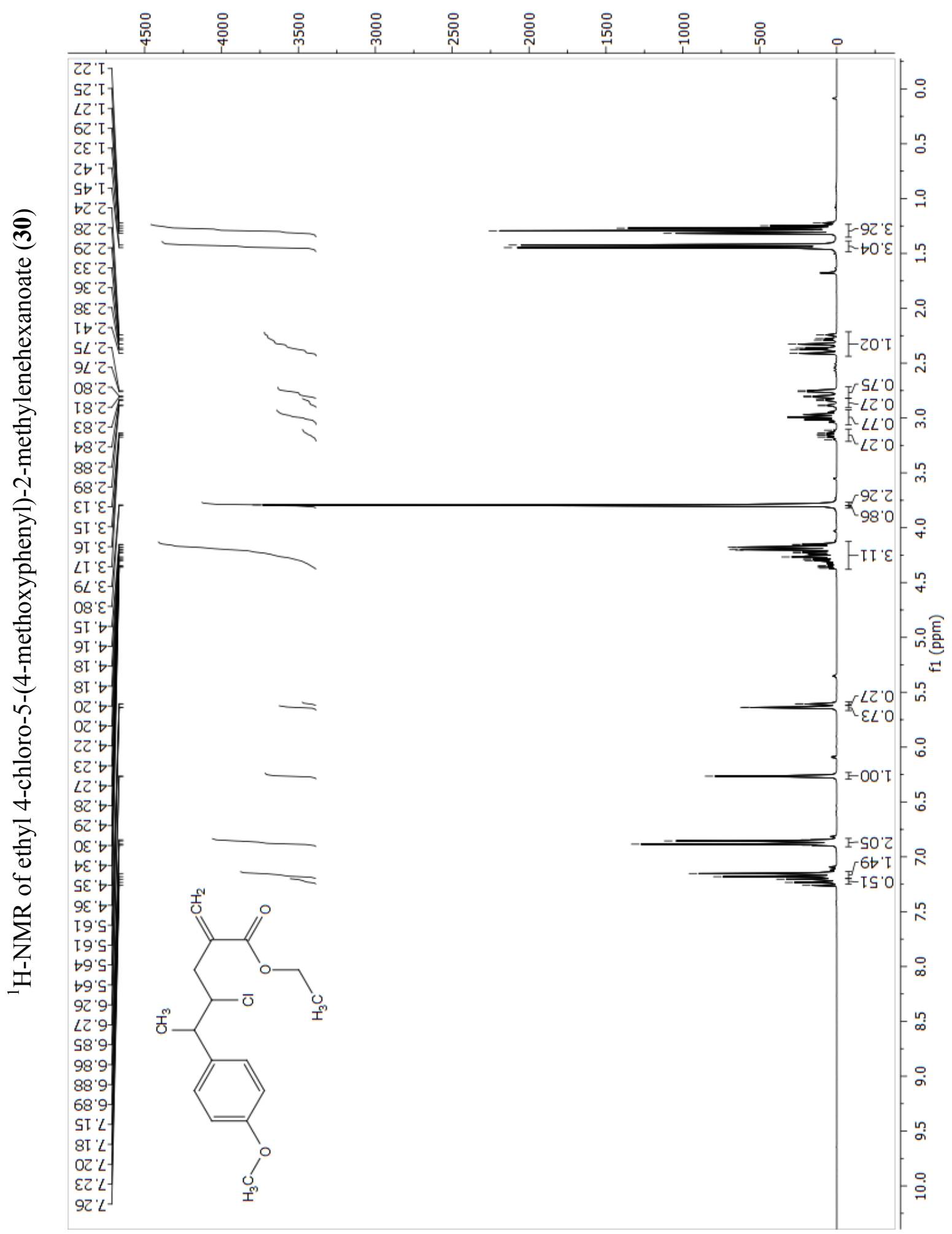




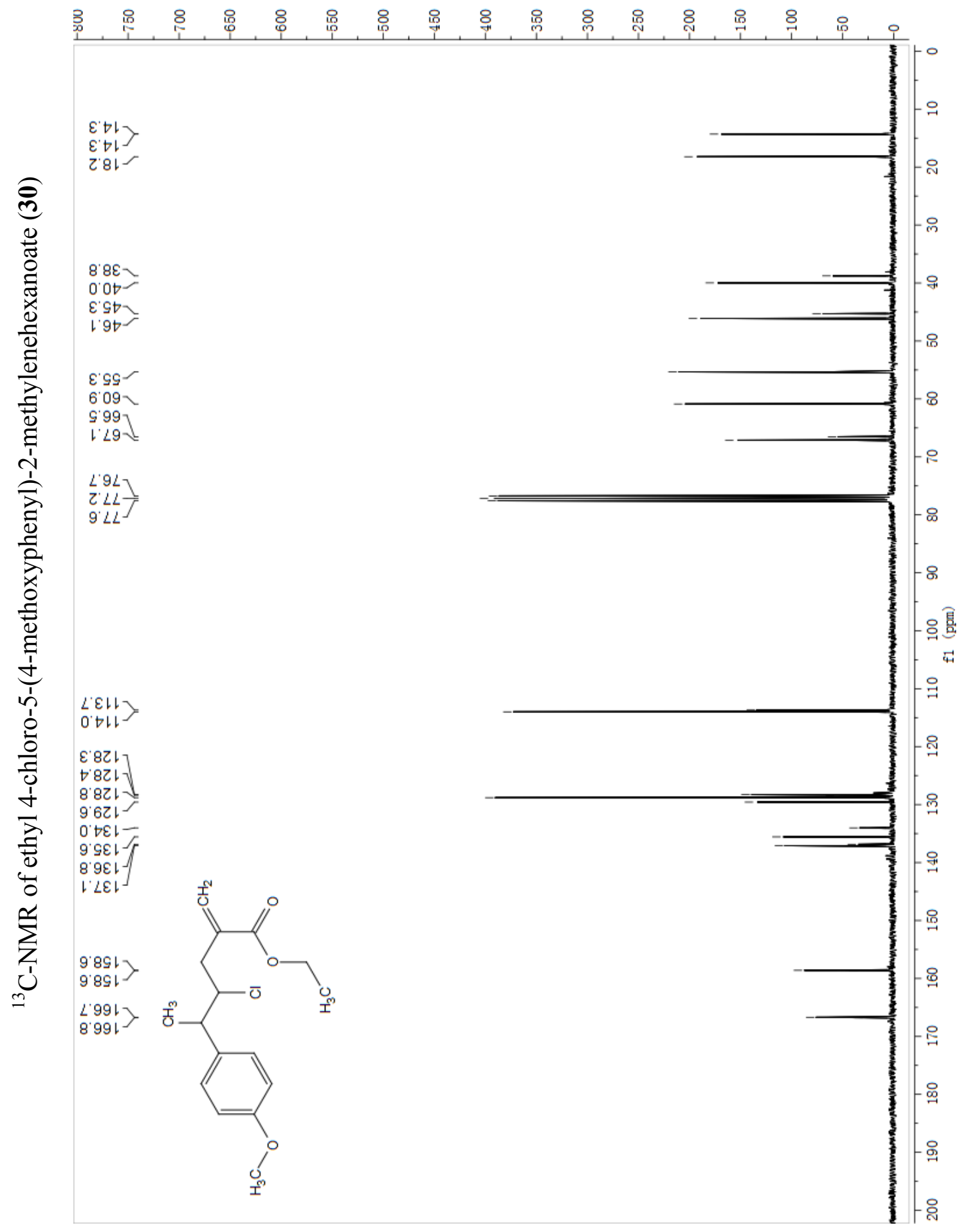




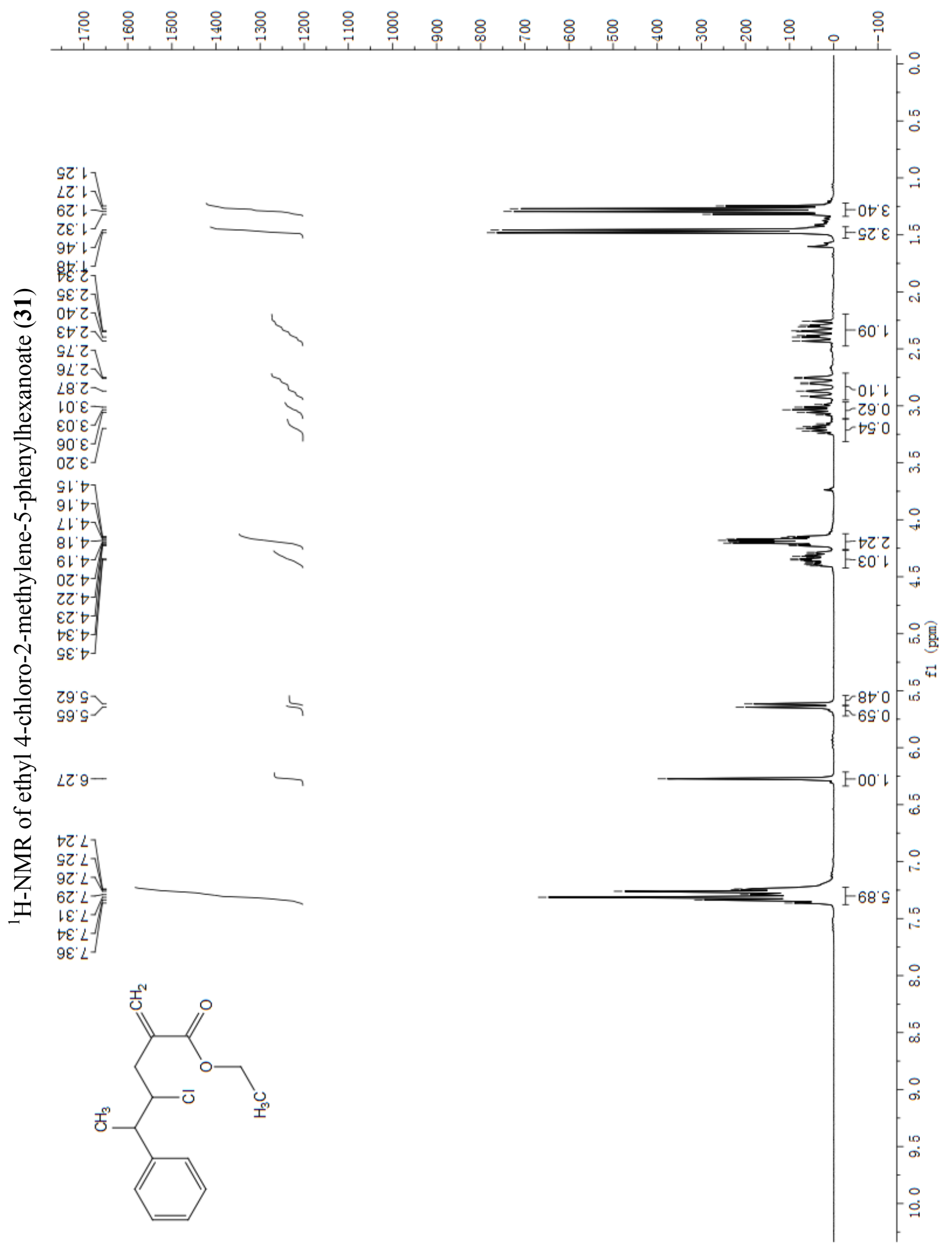




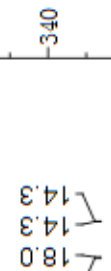

017
281
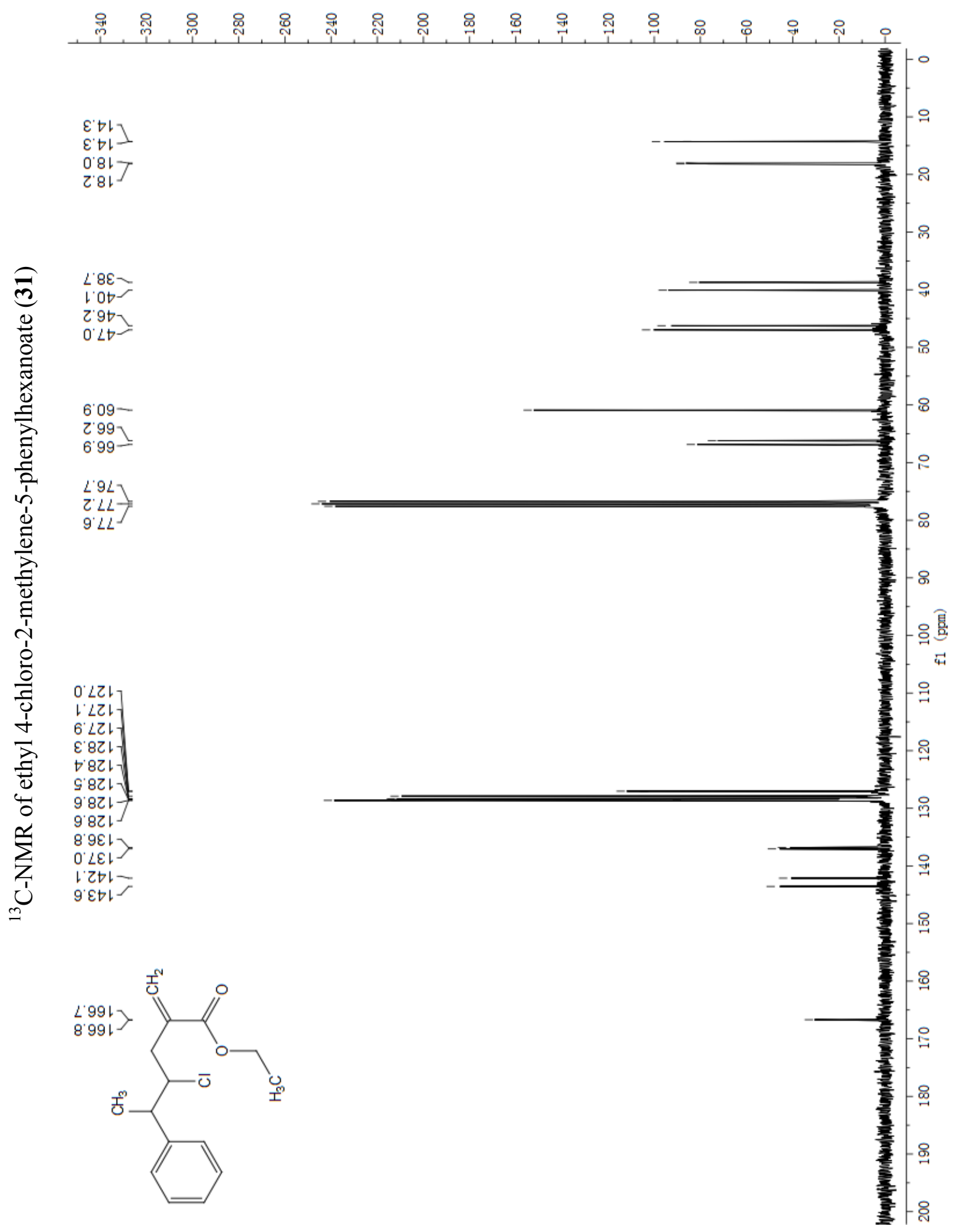


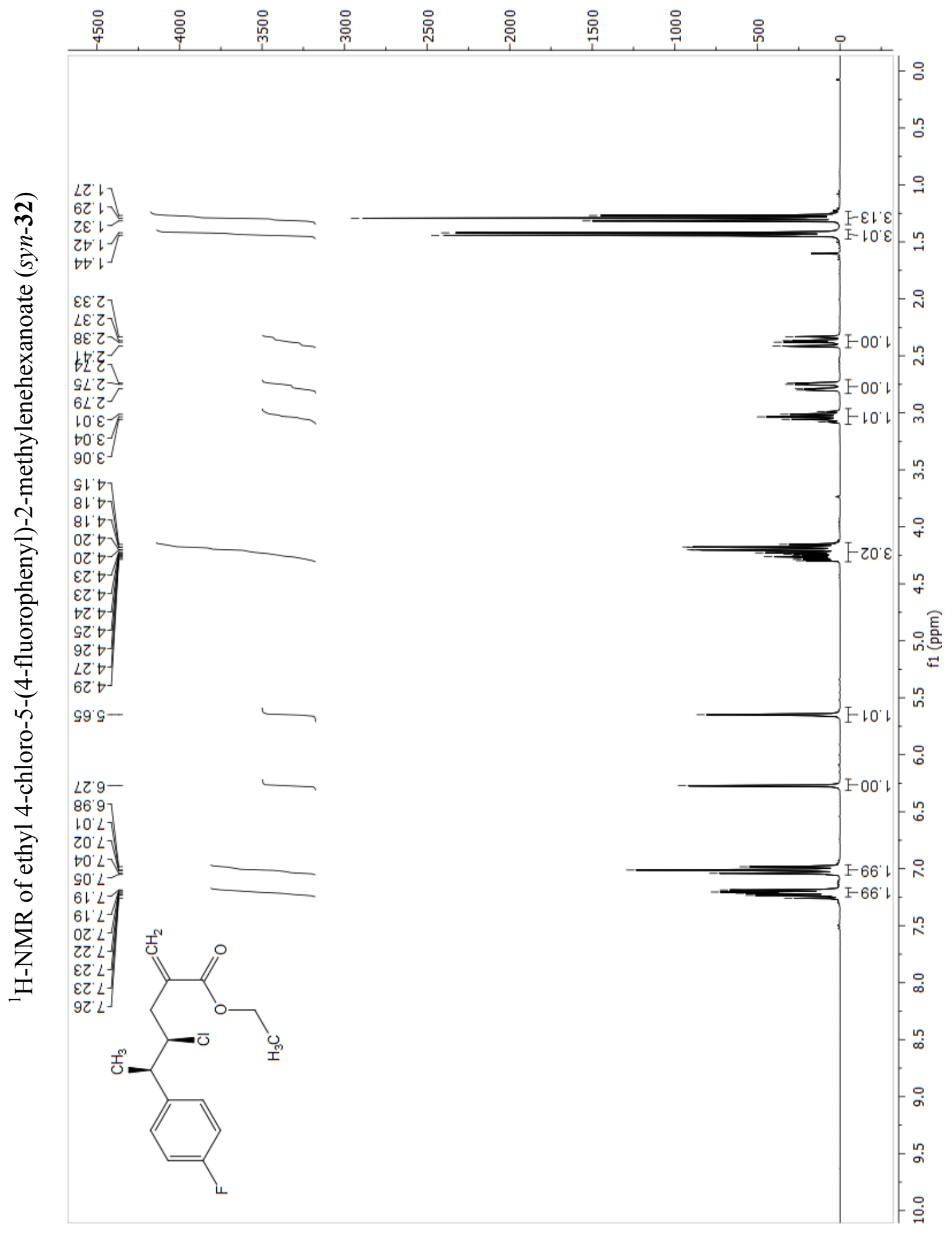




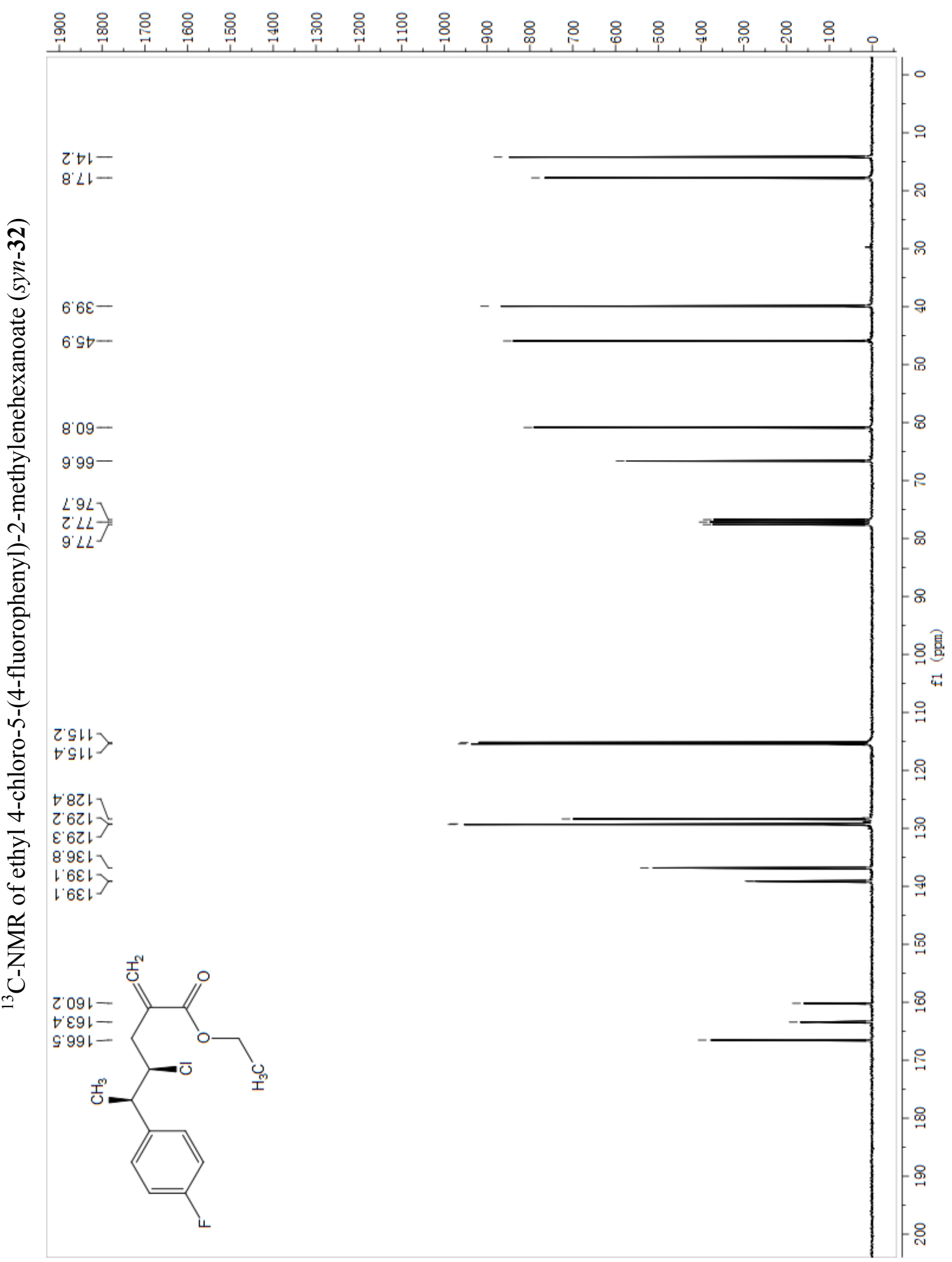




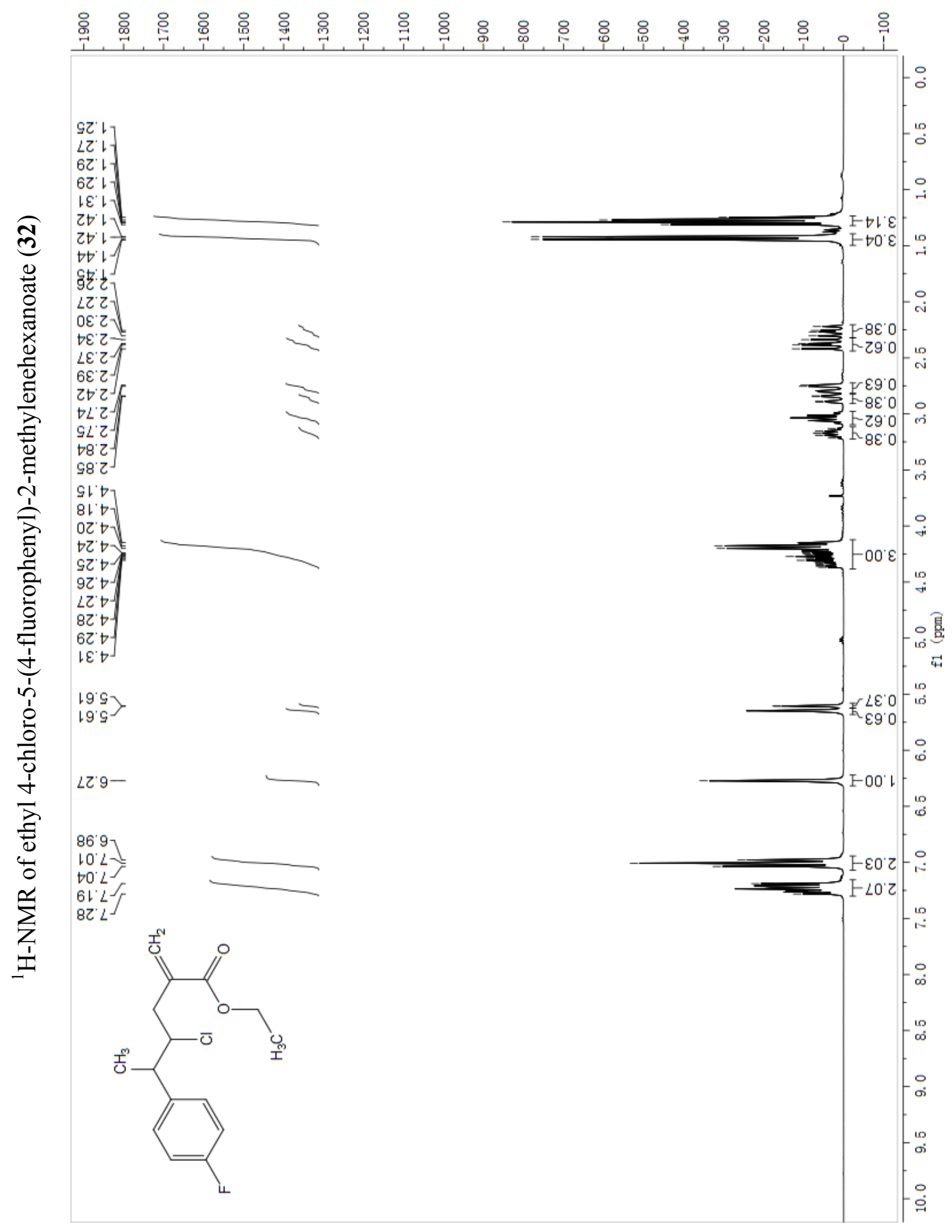




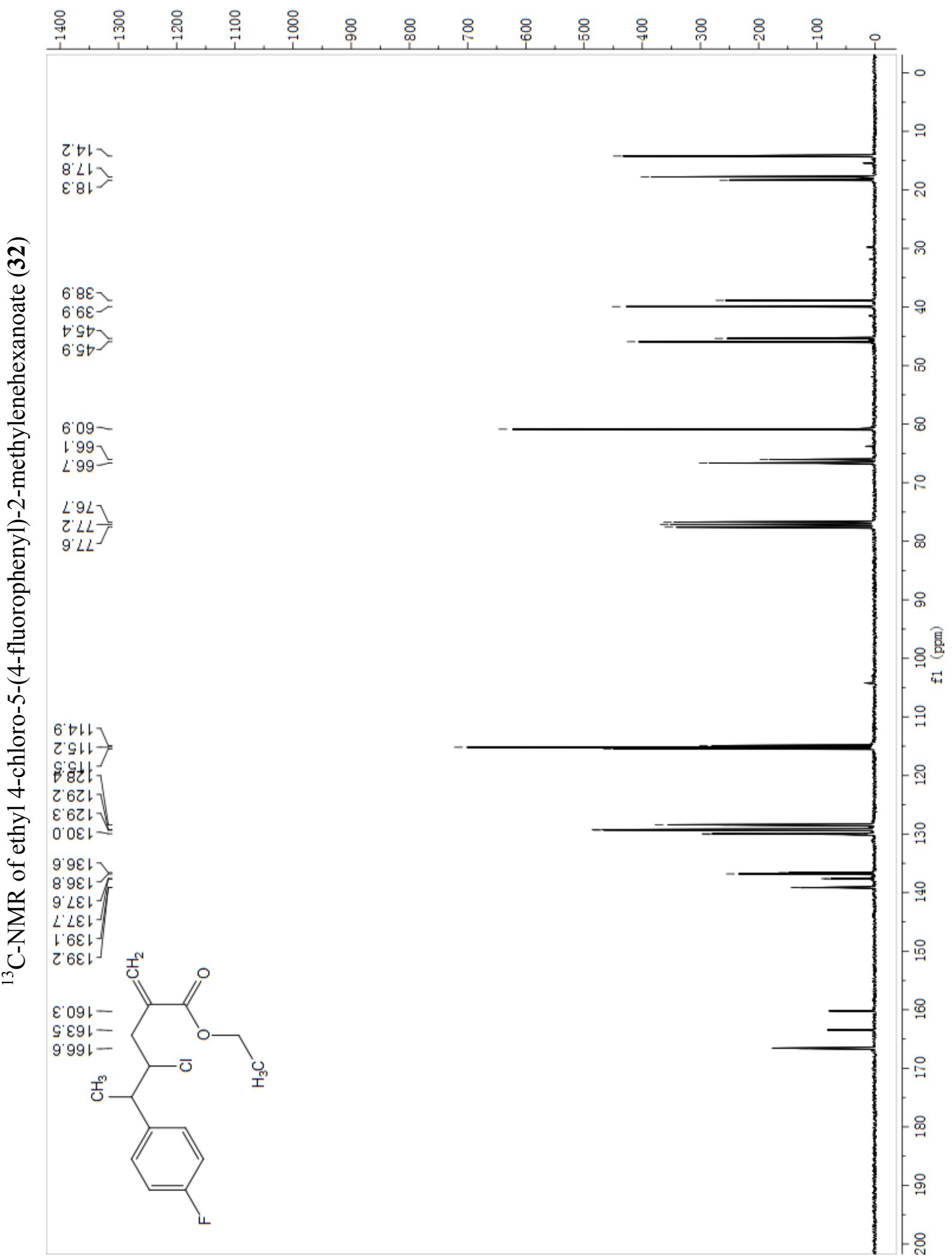




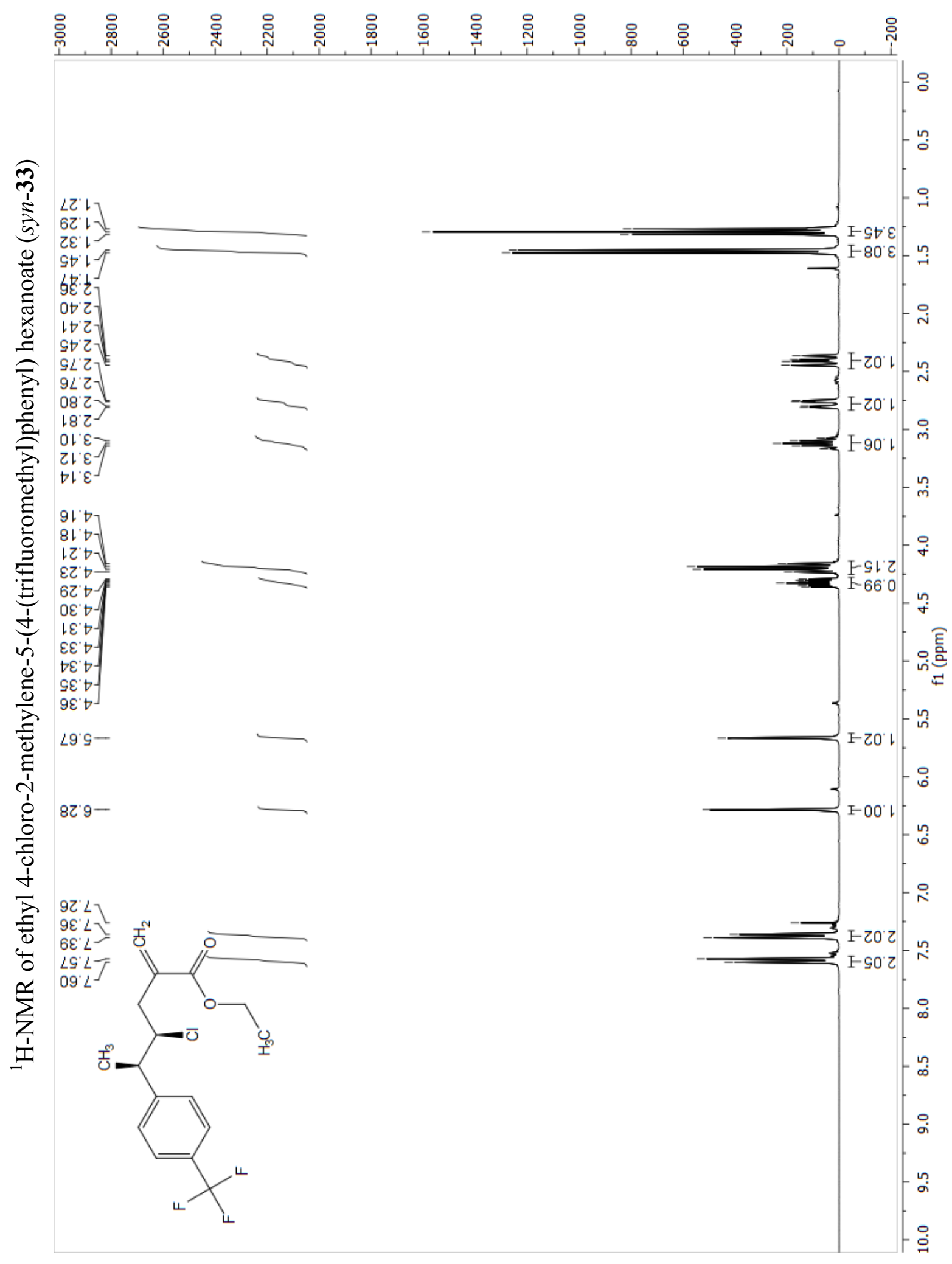




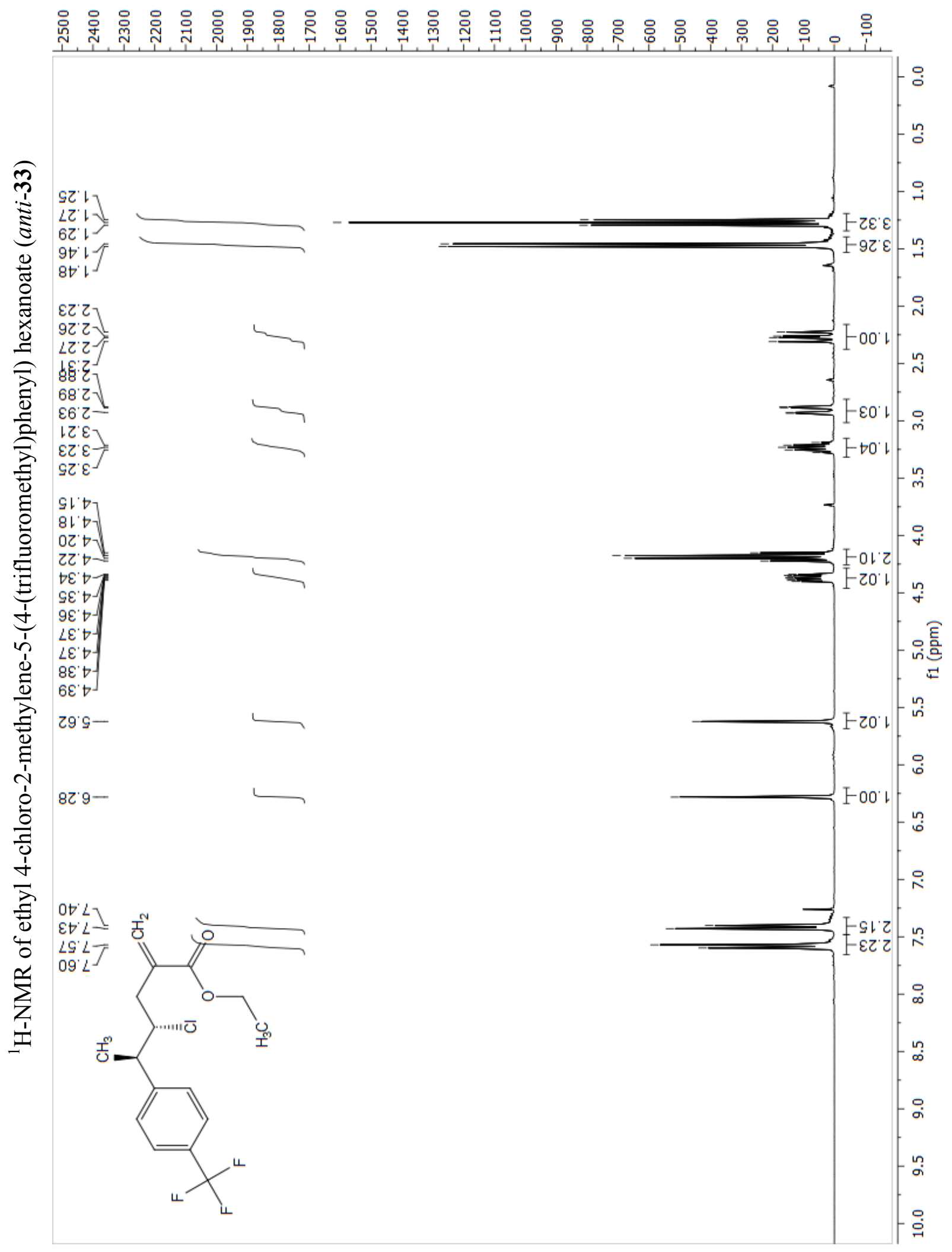




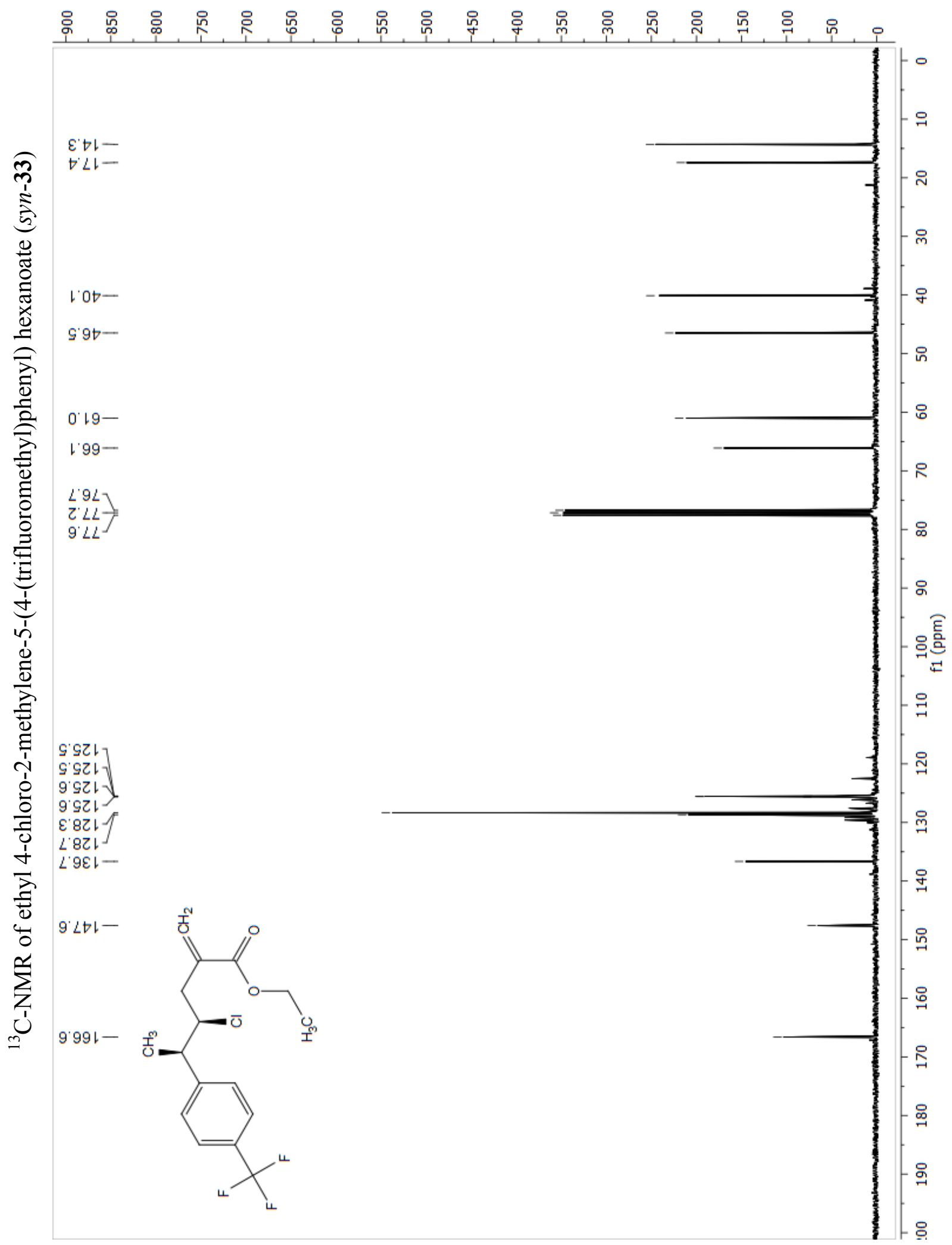




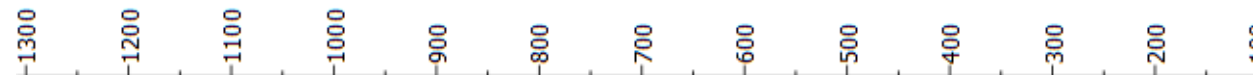
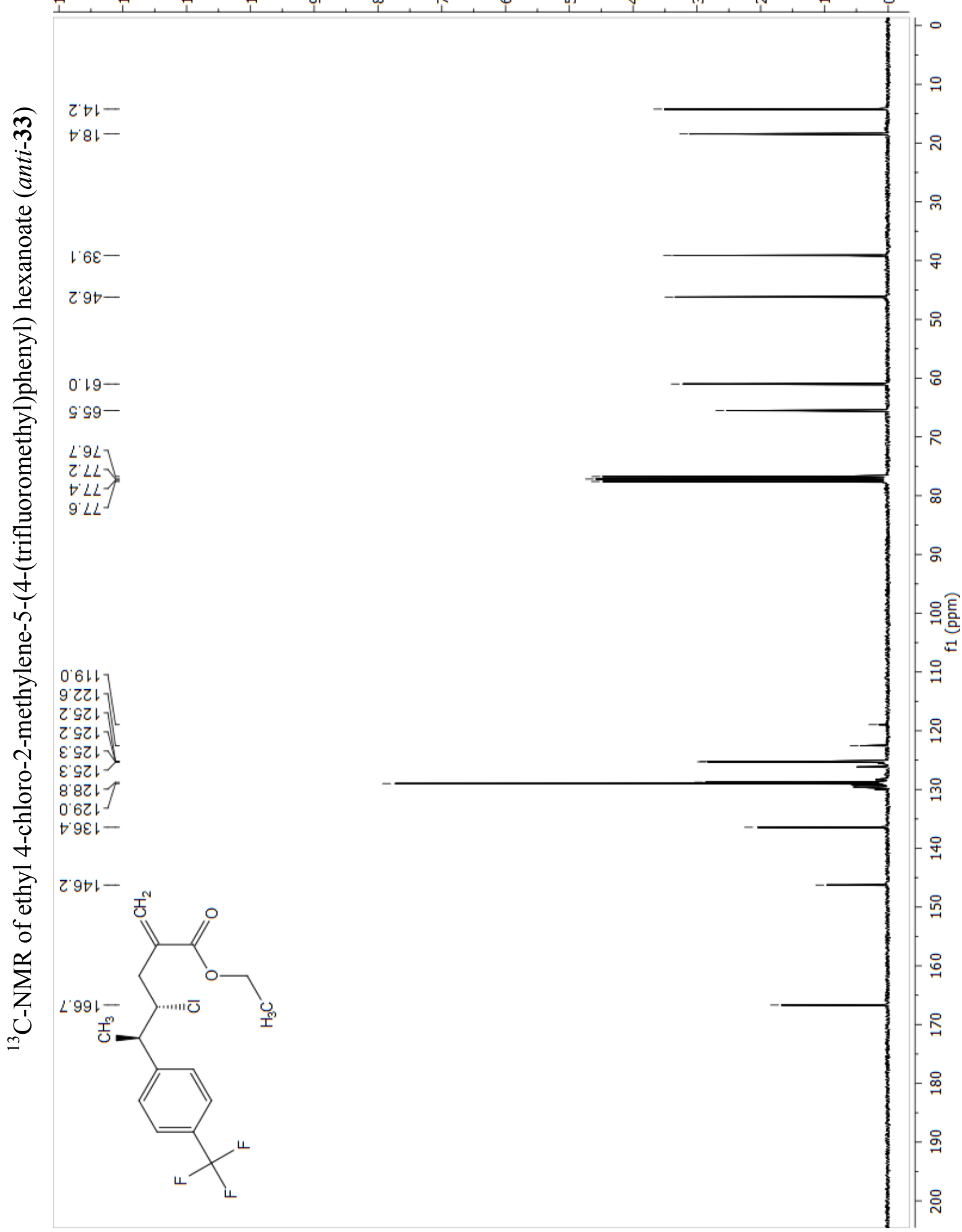


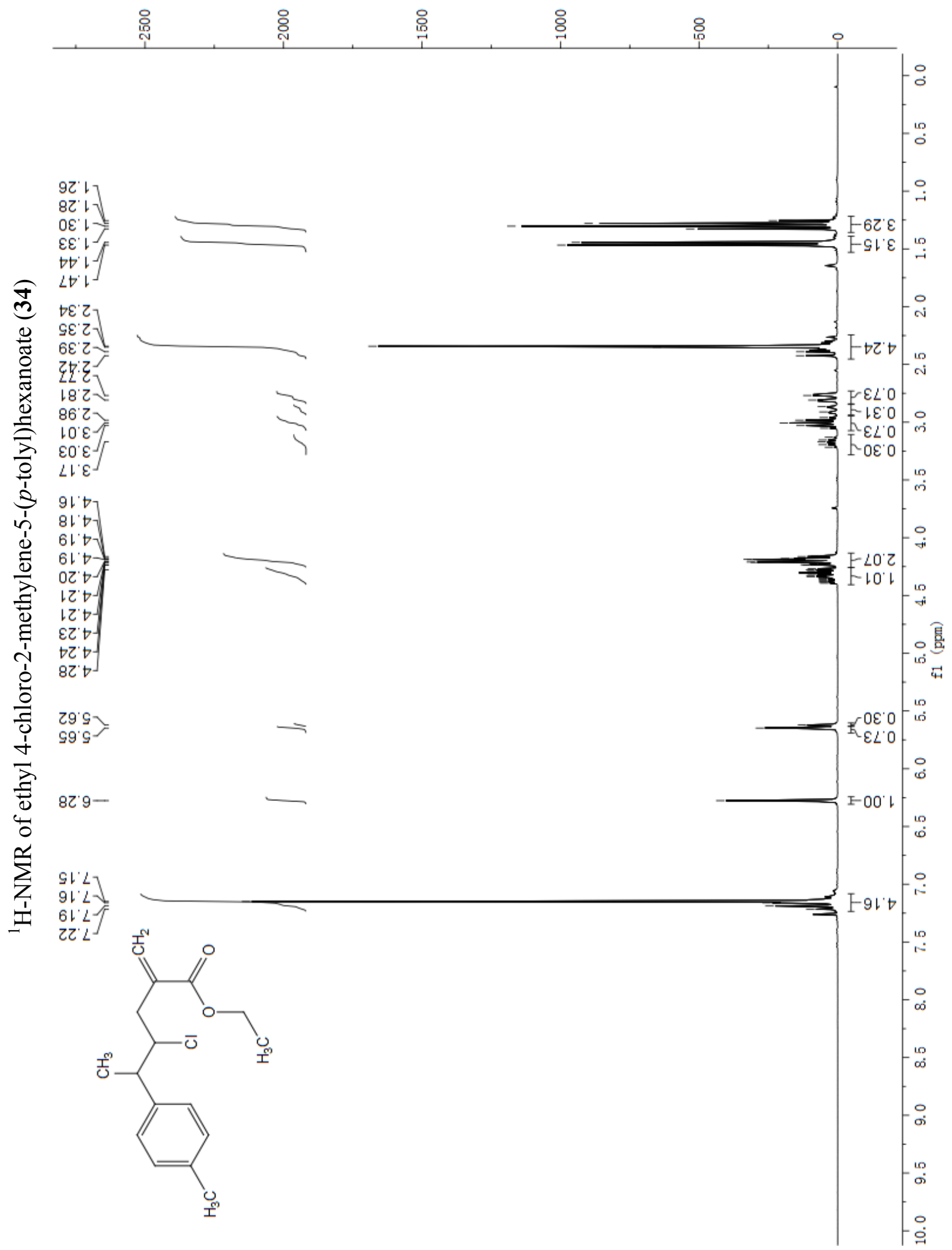




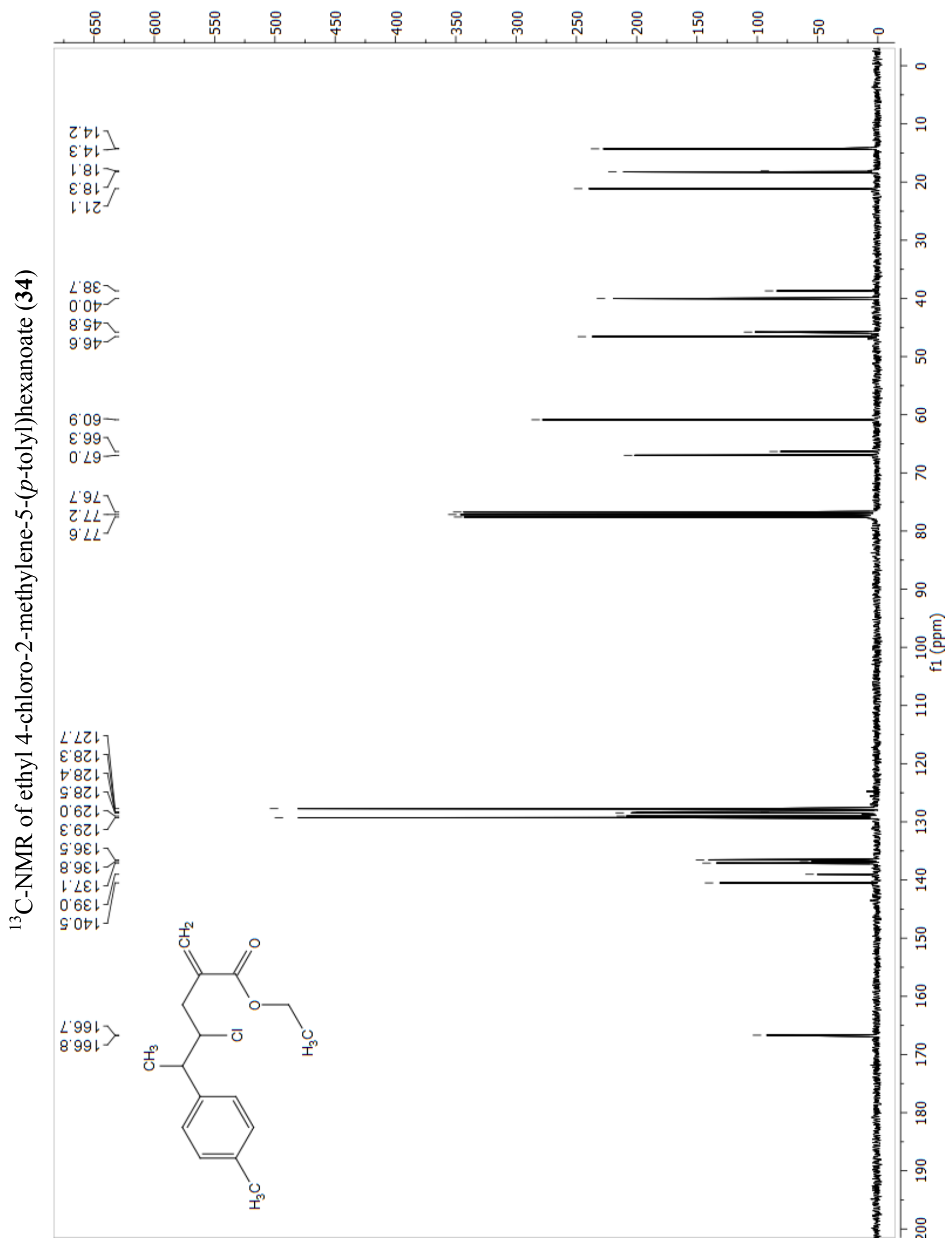




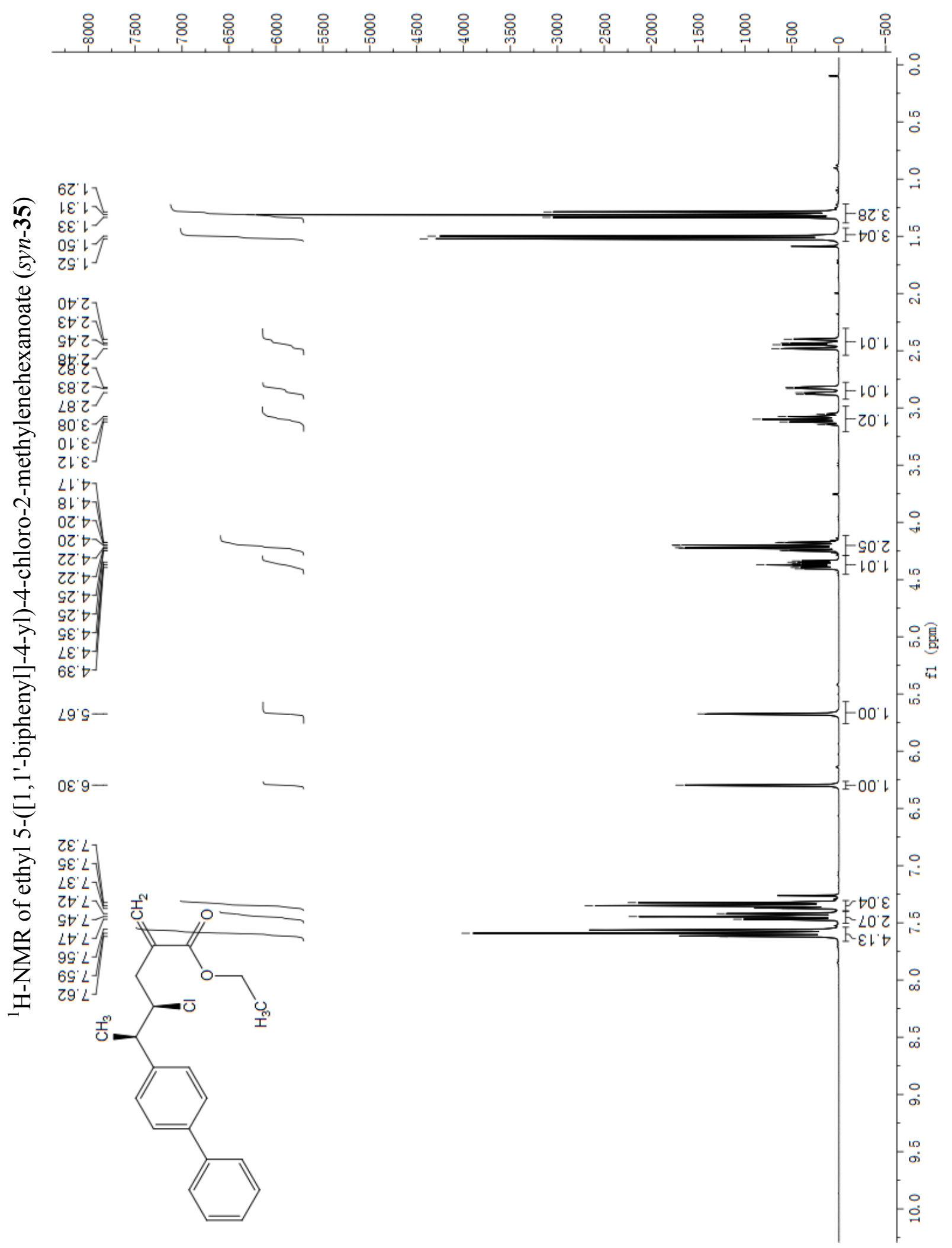




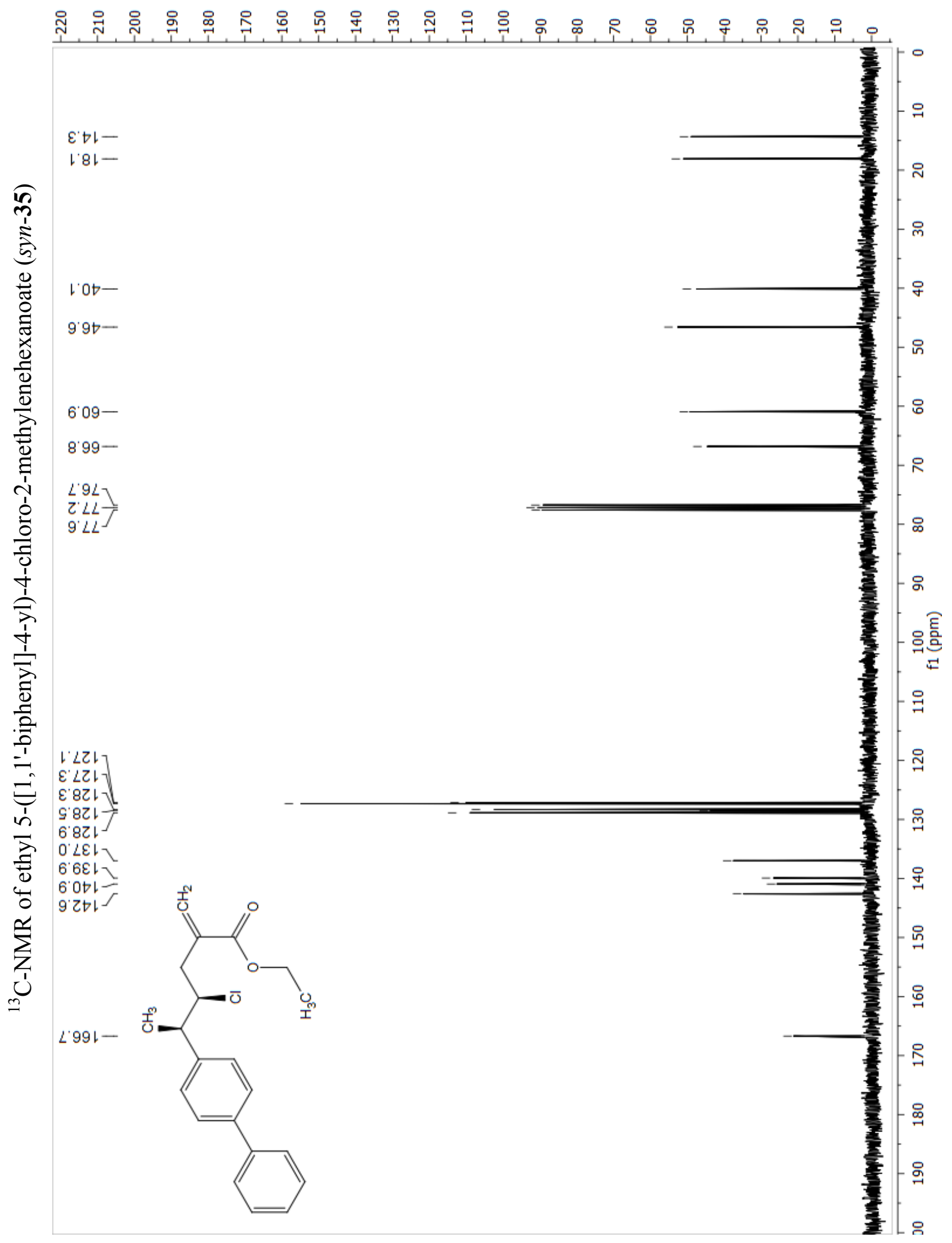




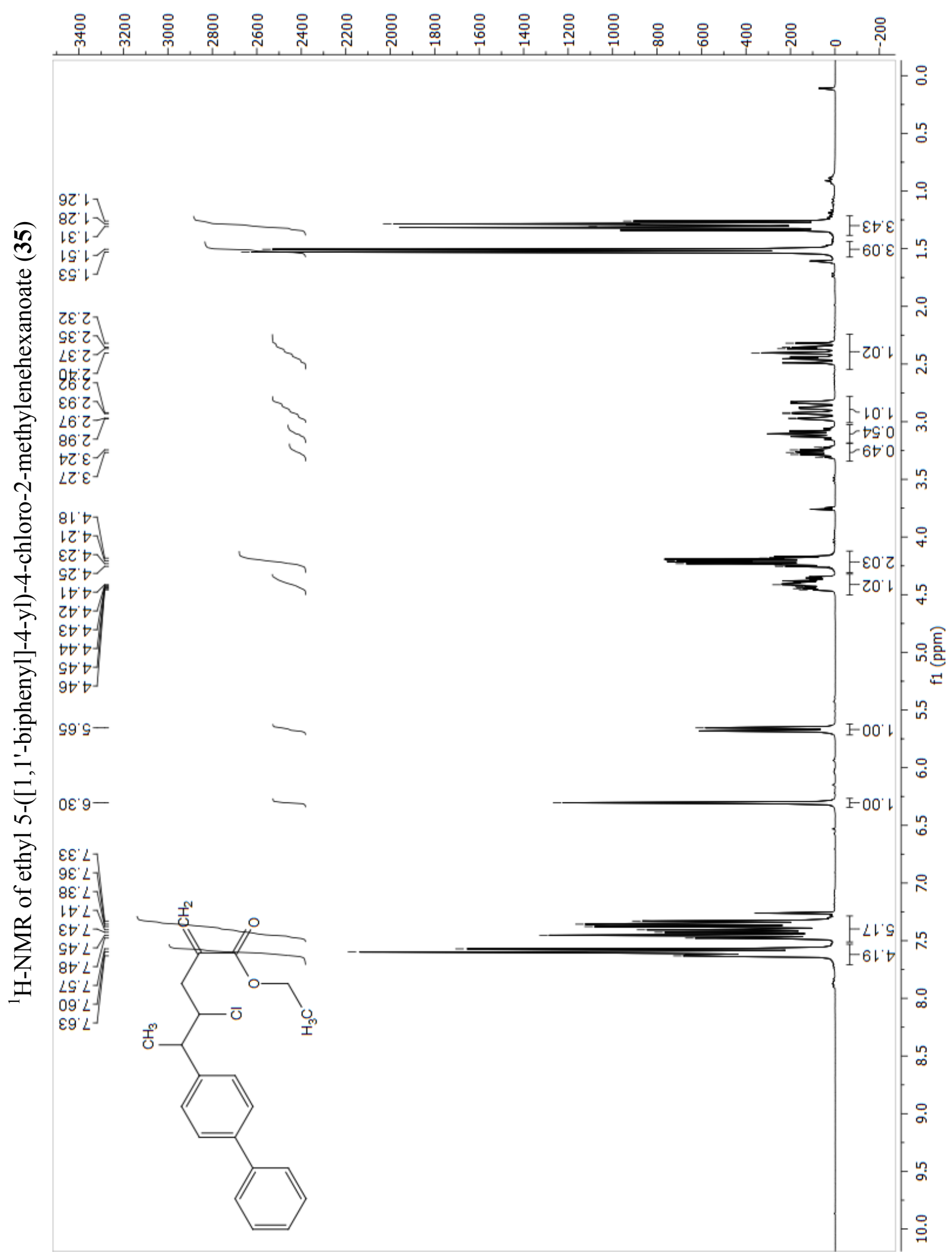




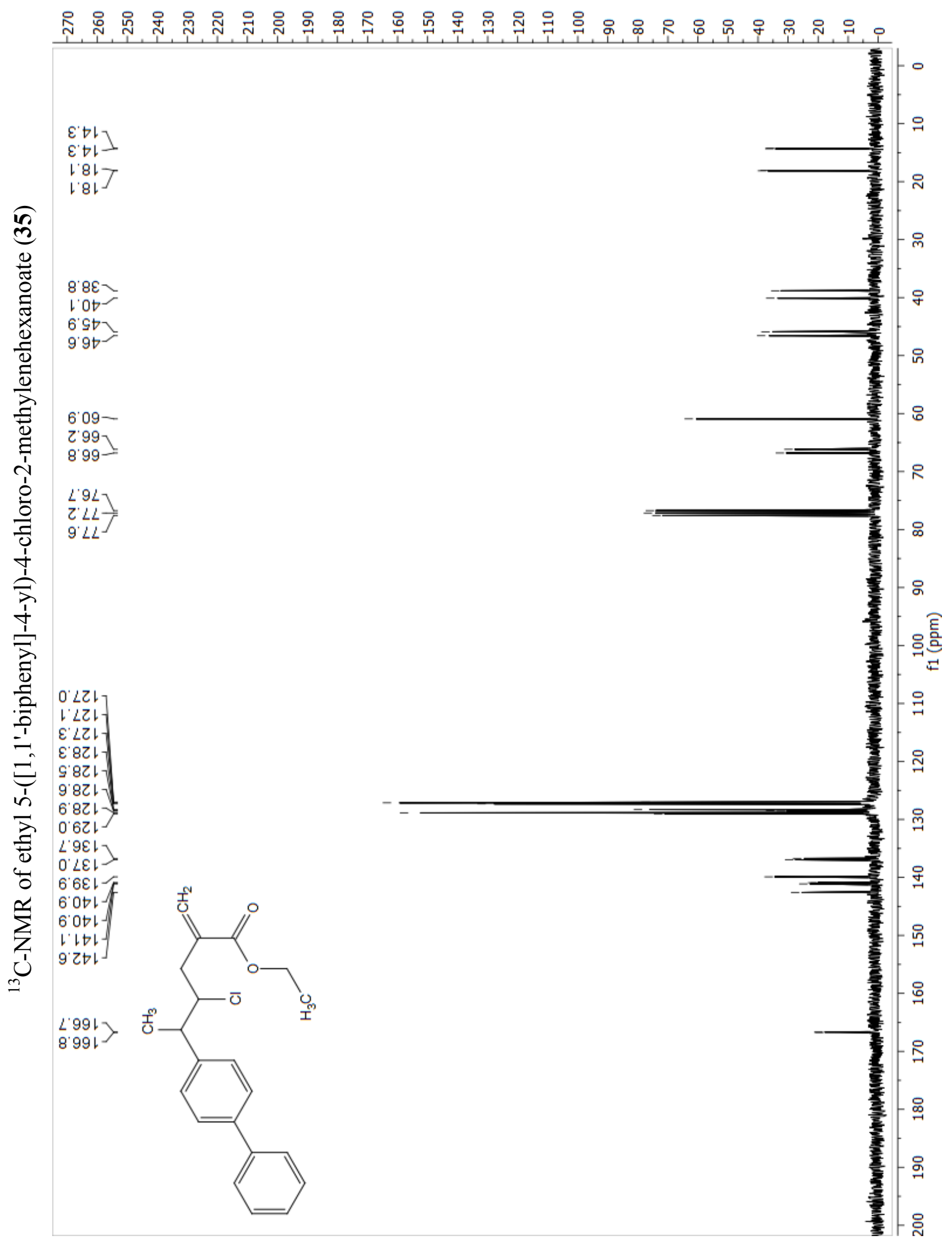




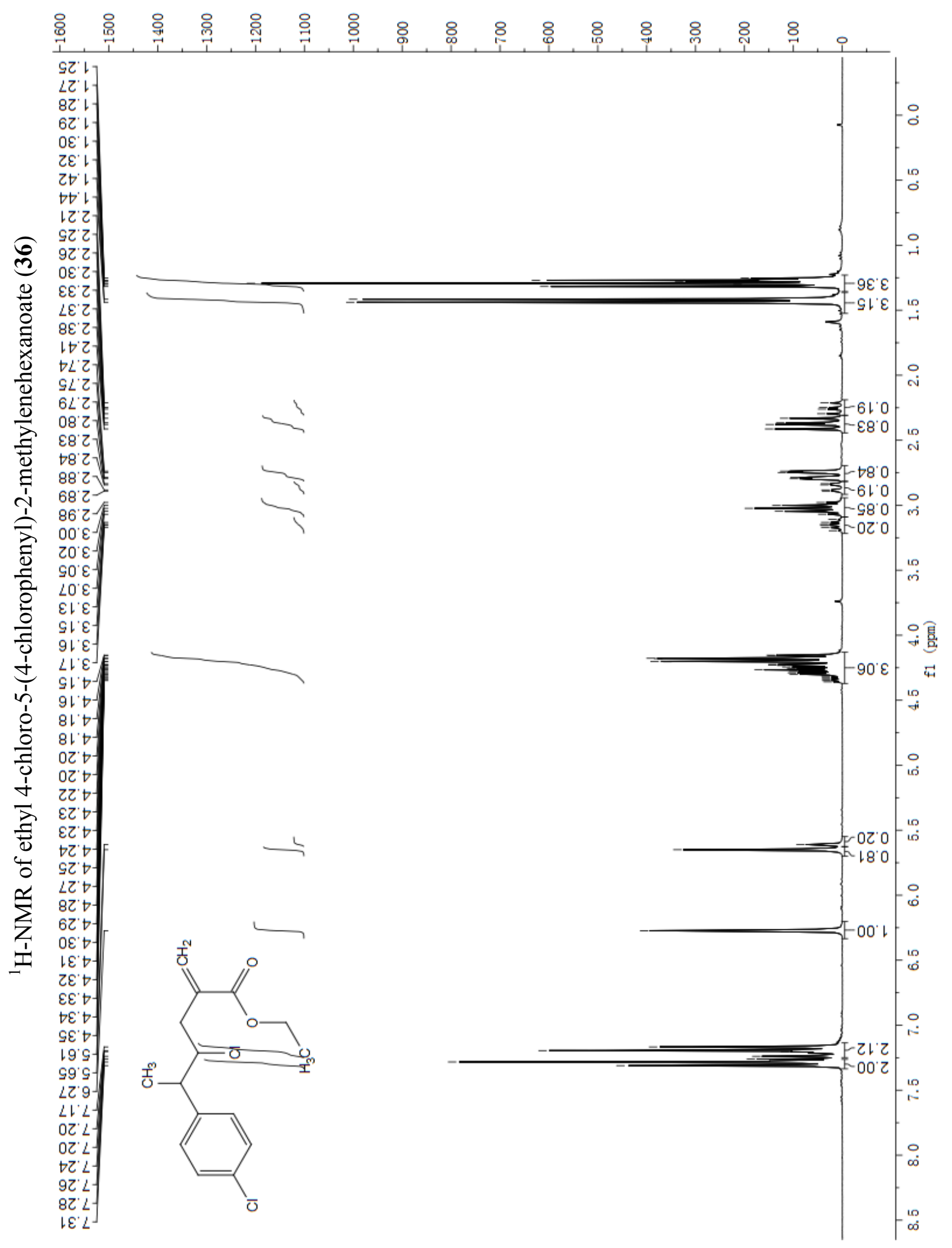




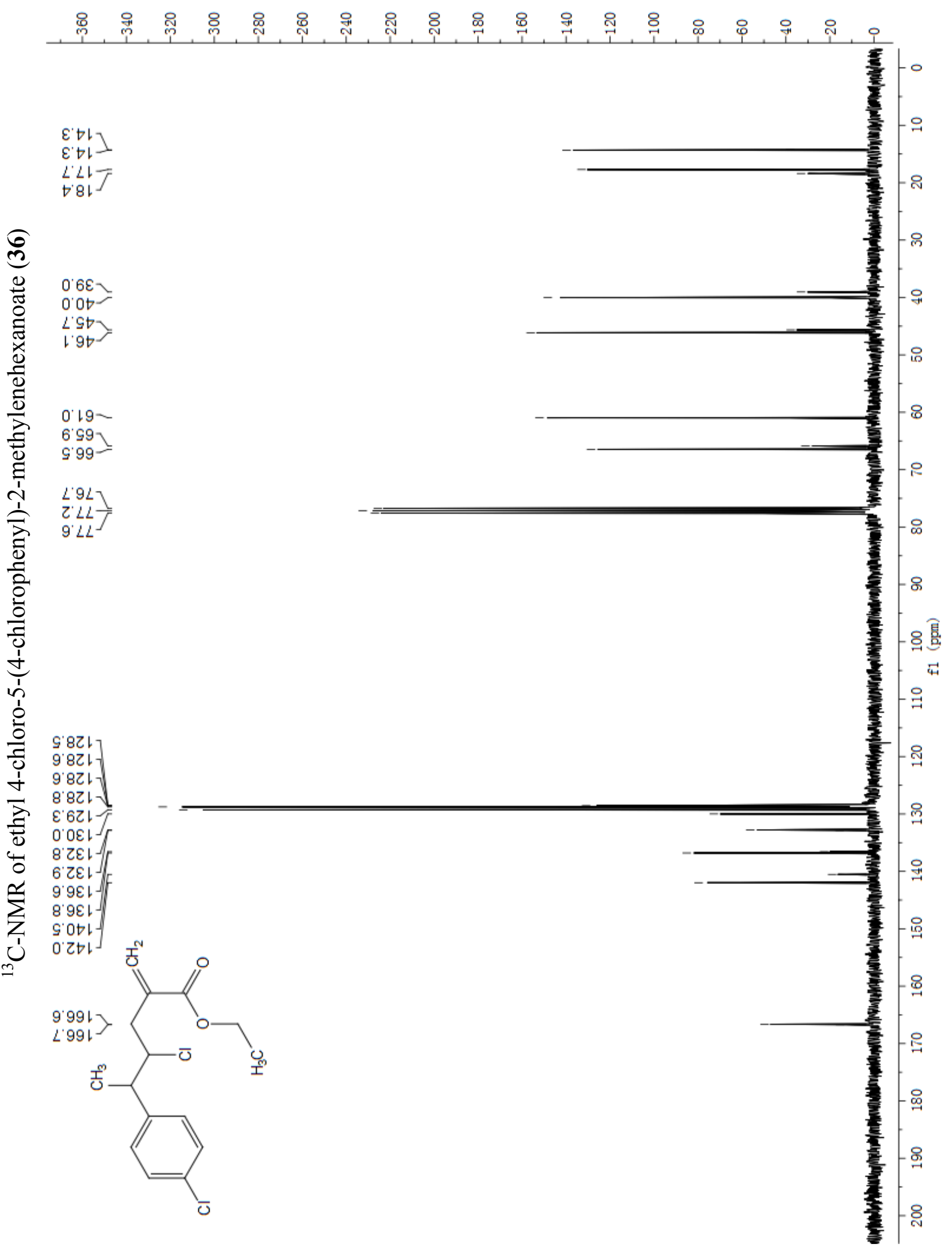




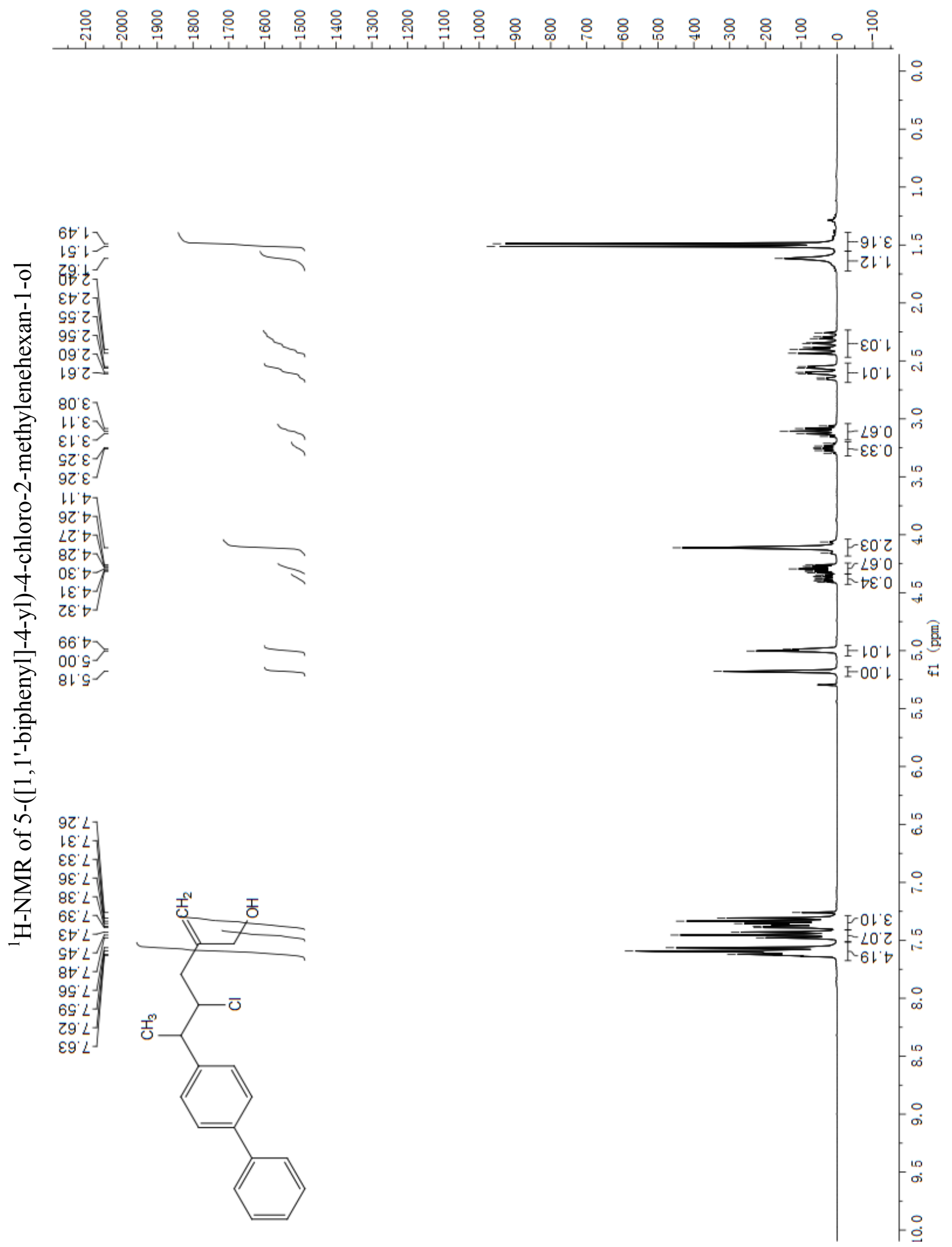




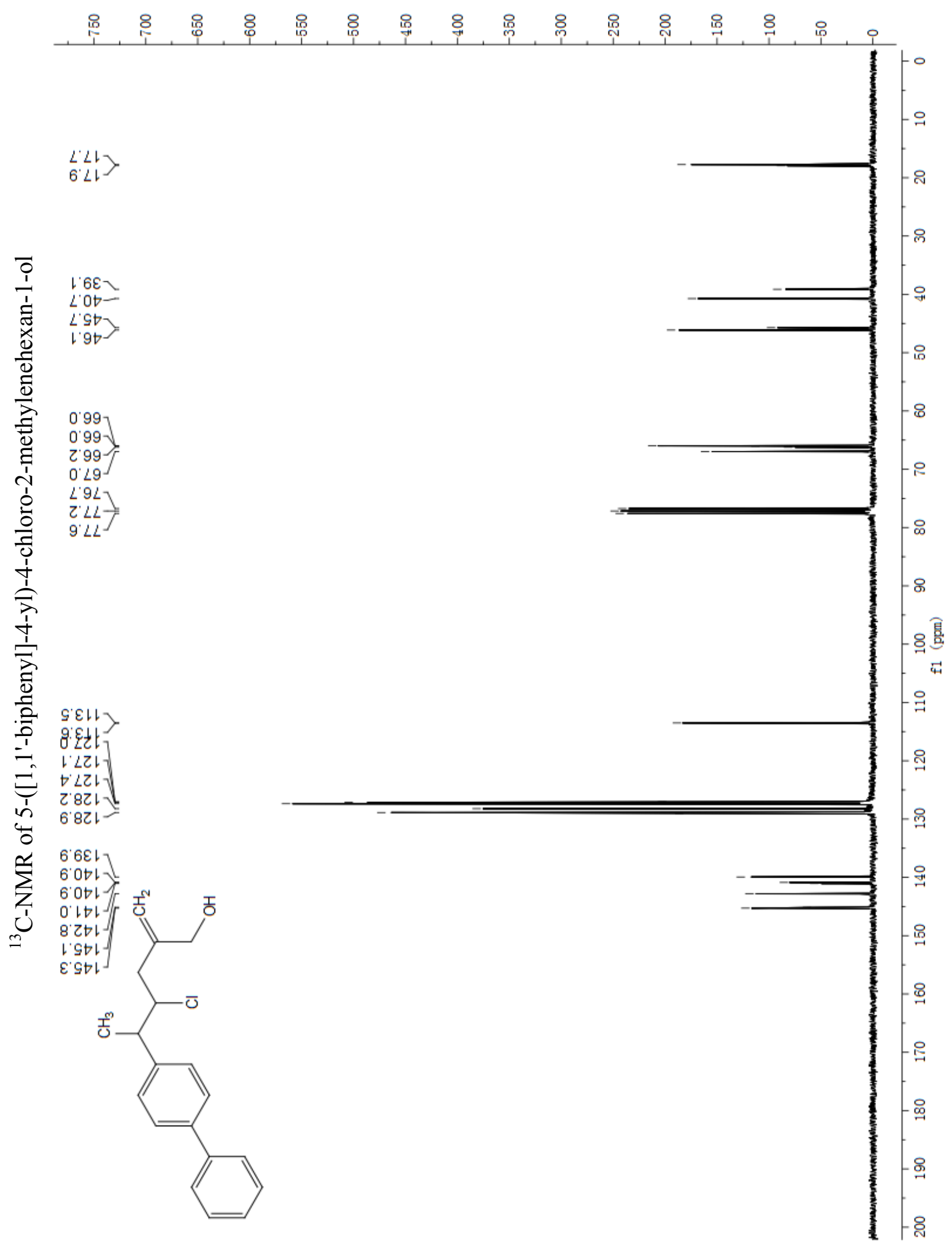




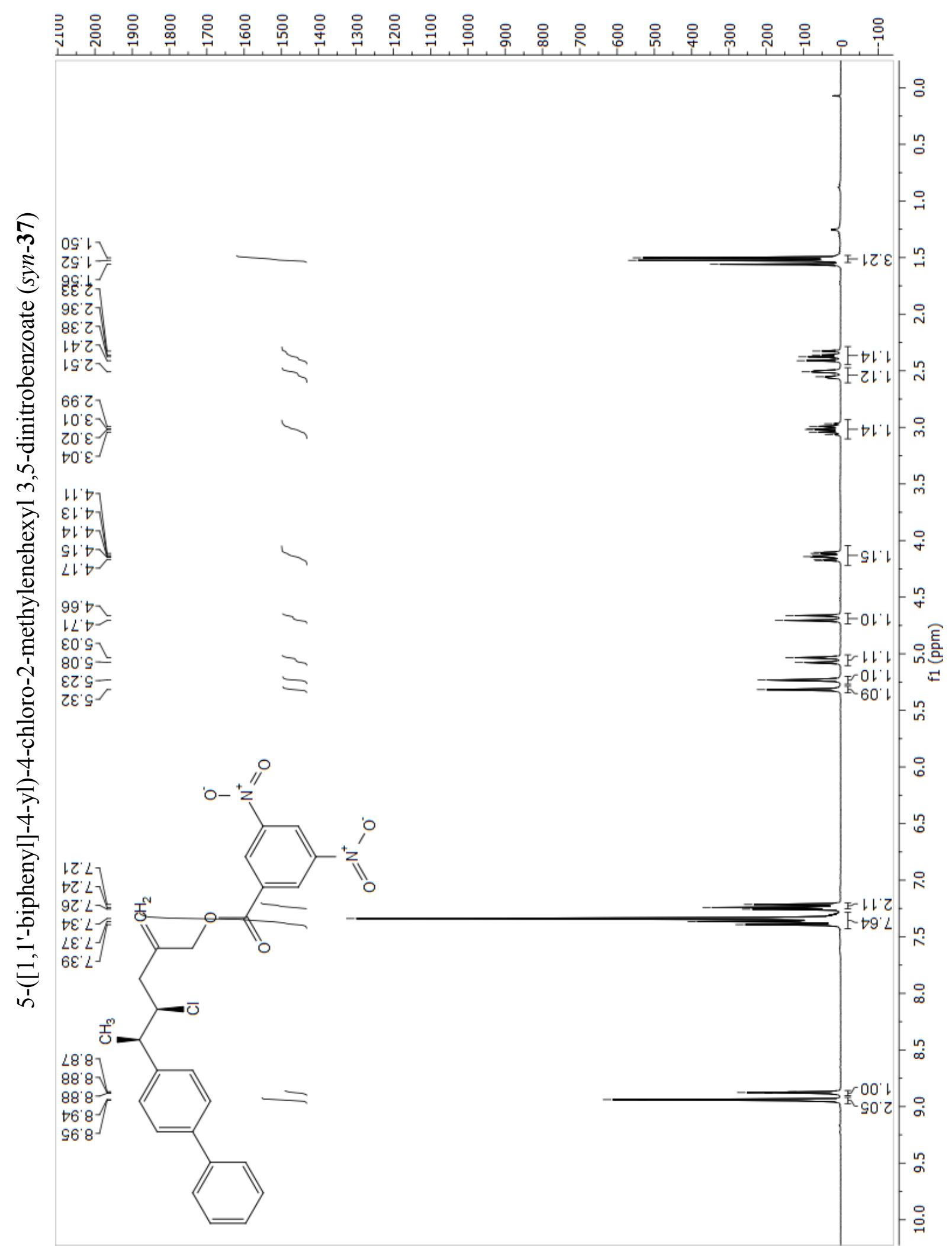




$$
\frac{Z}{1}
$$




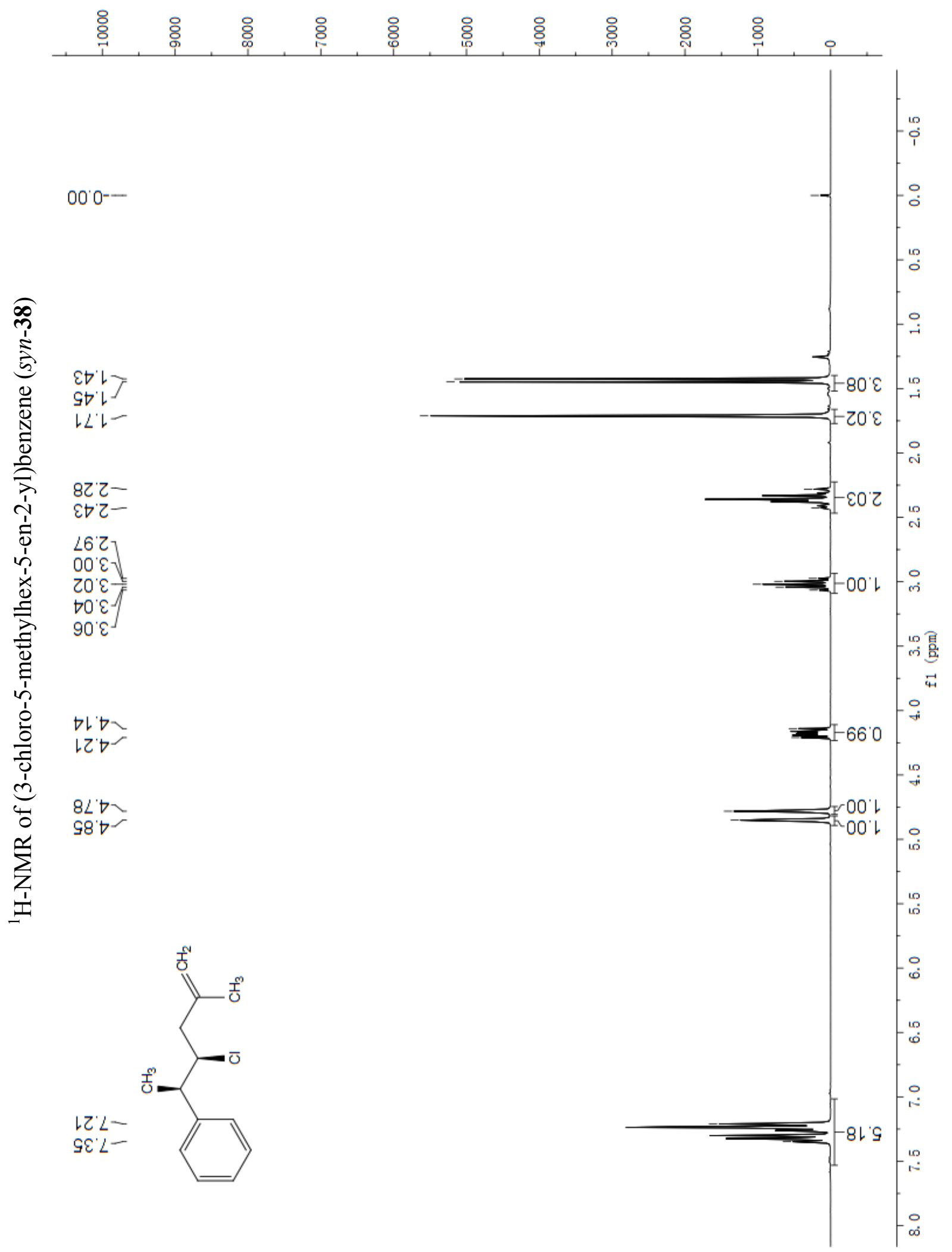




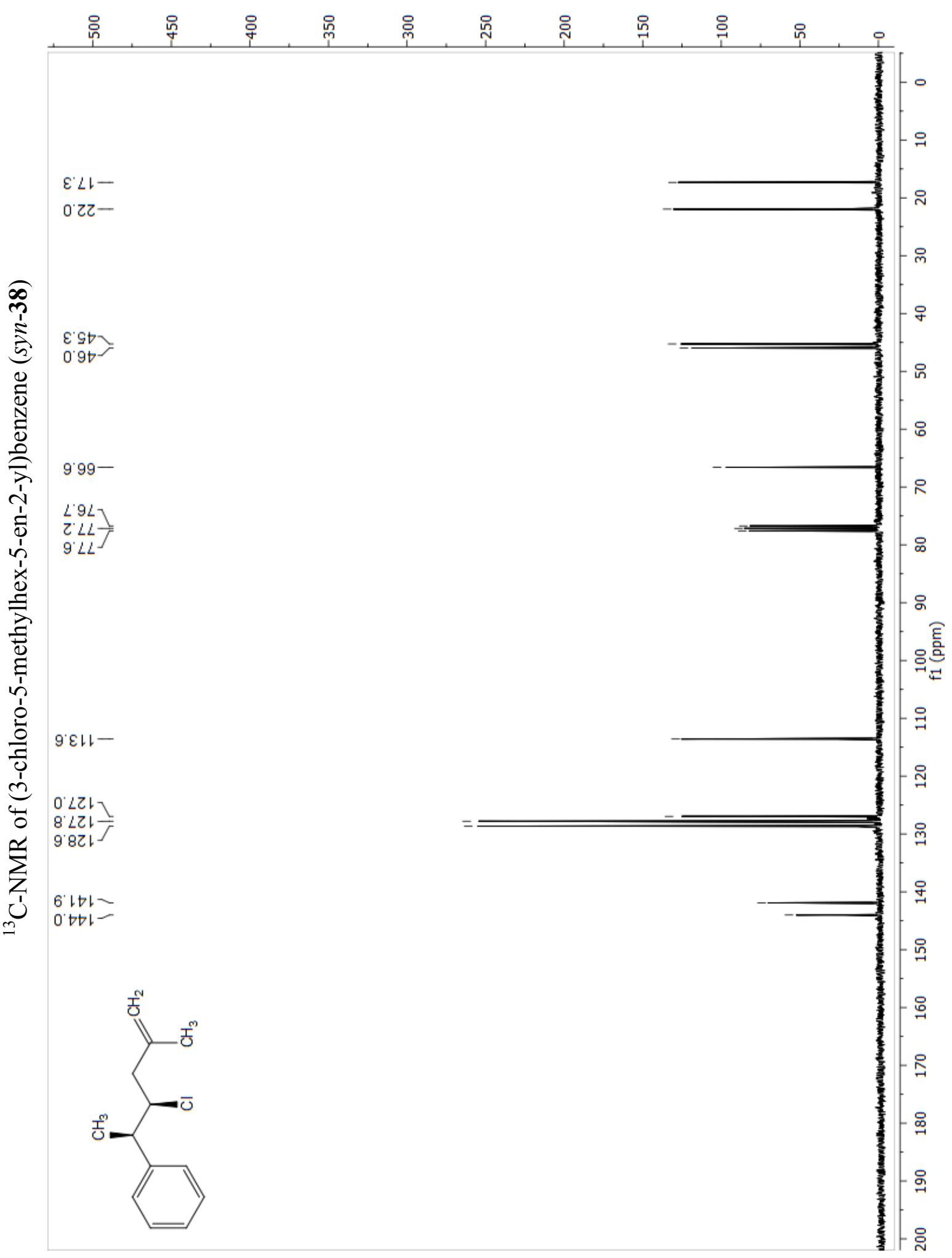




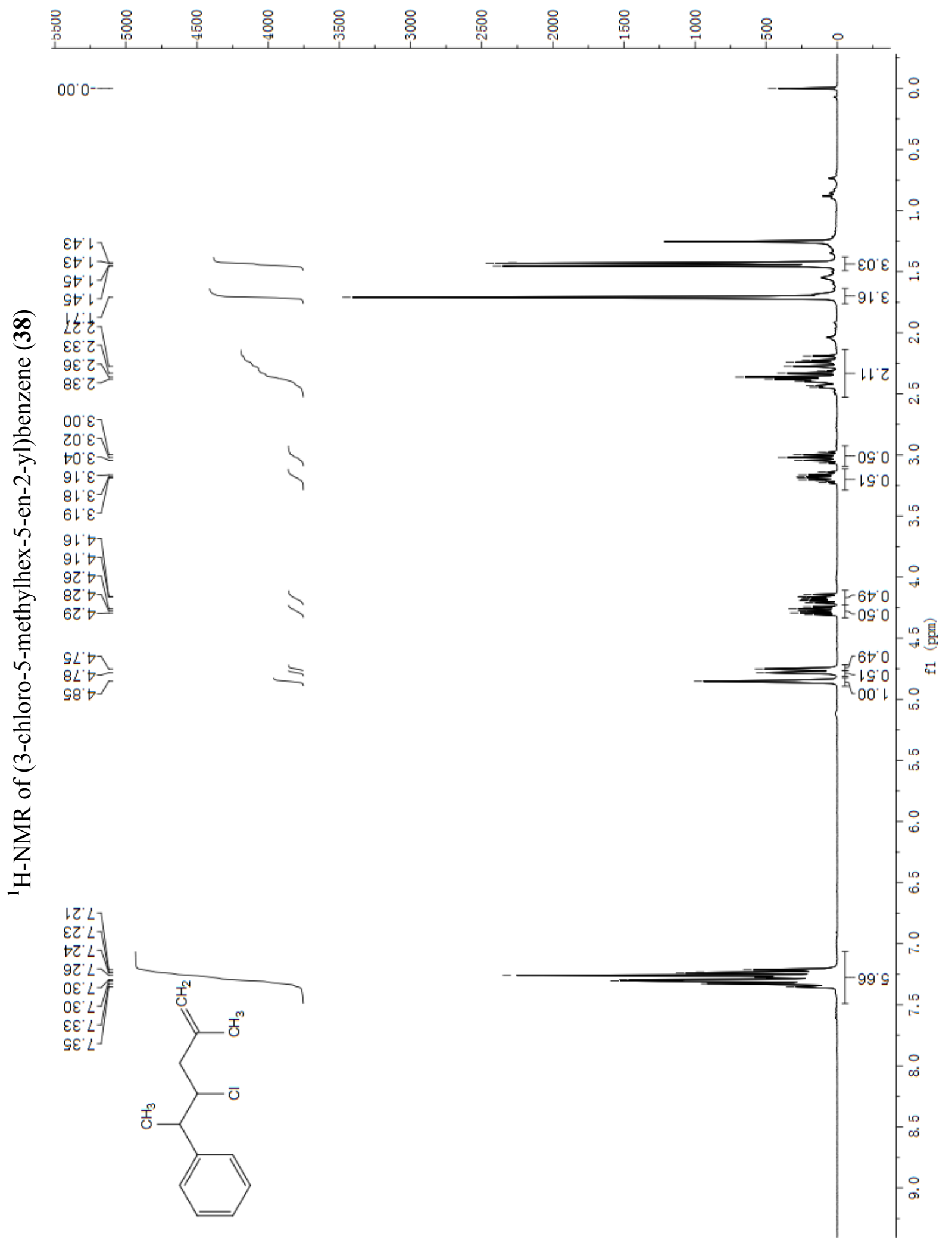




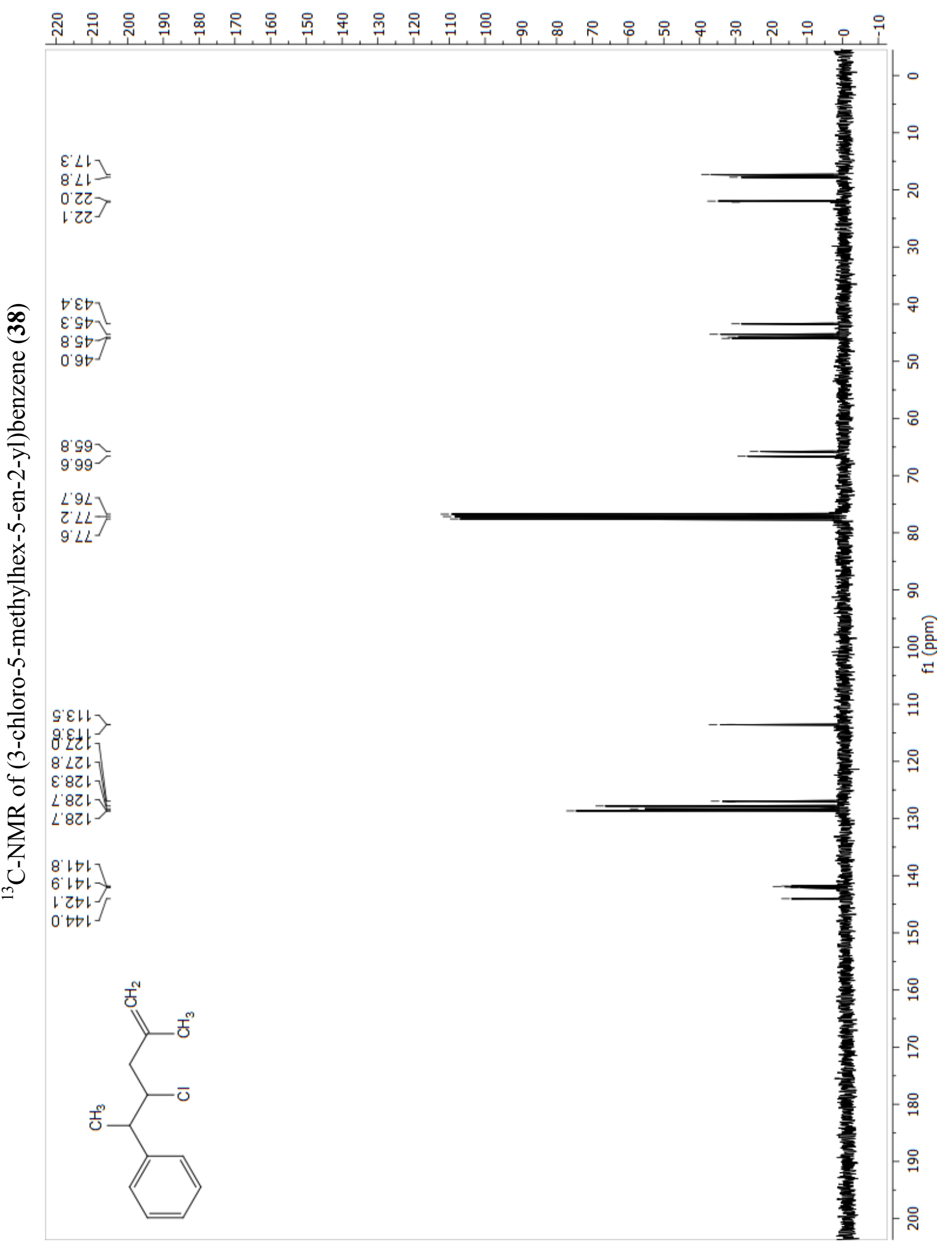




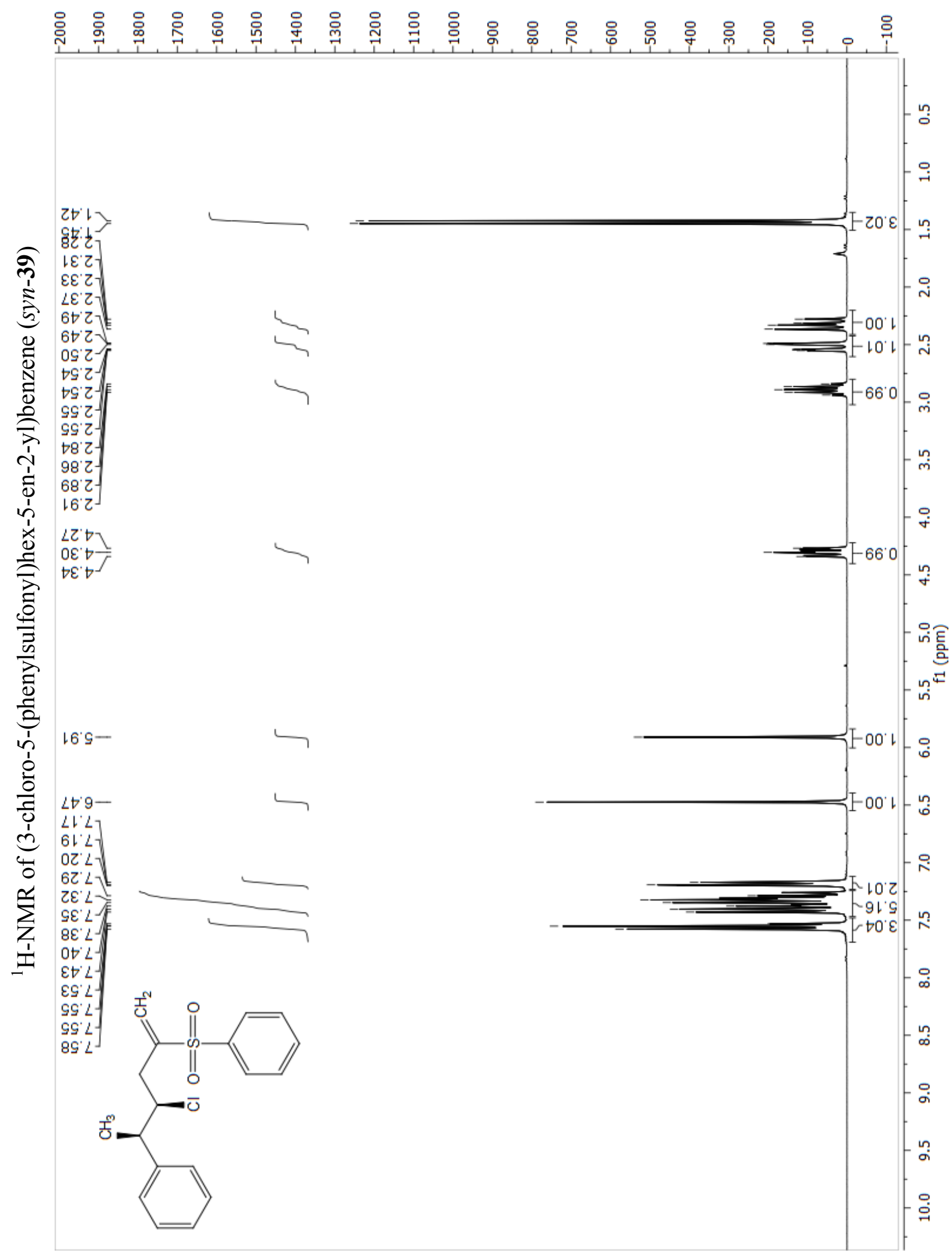




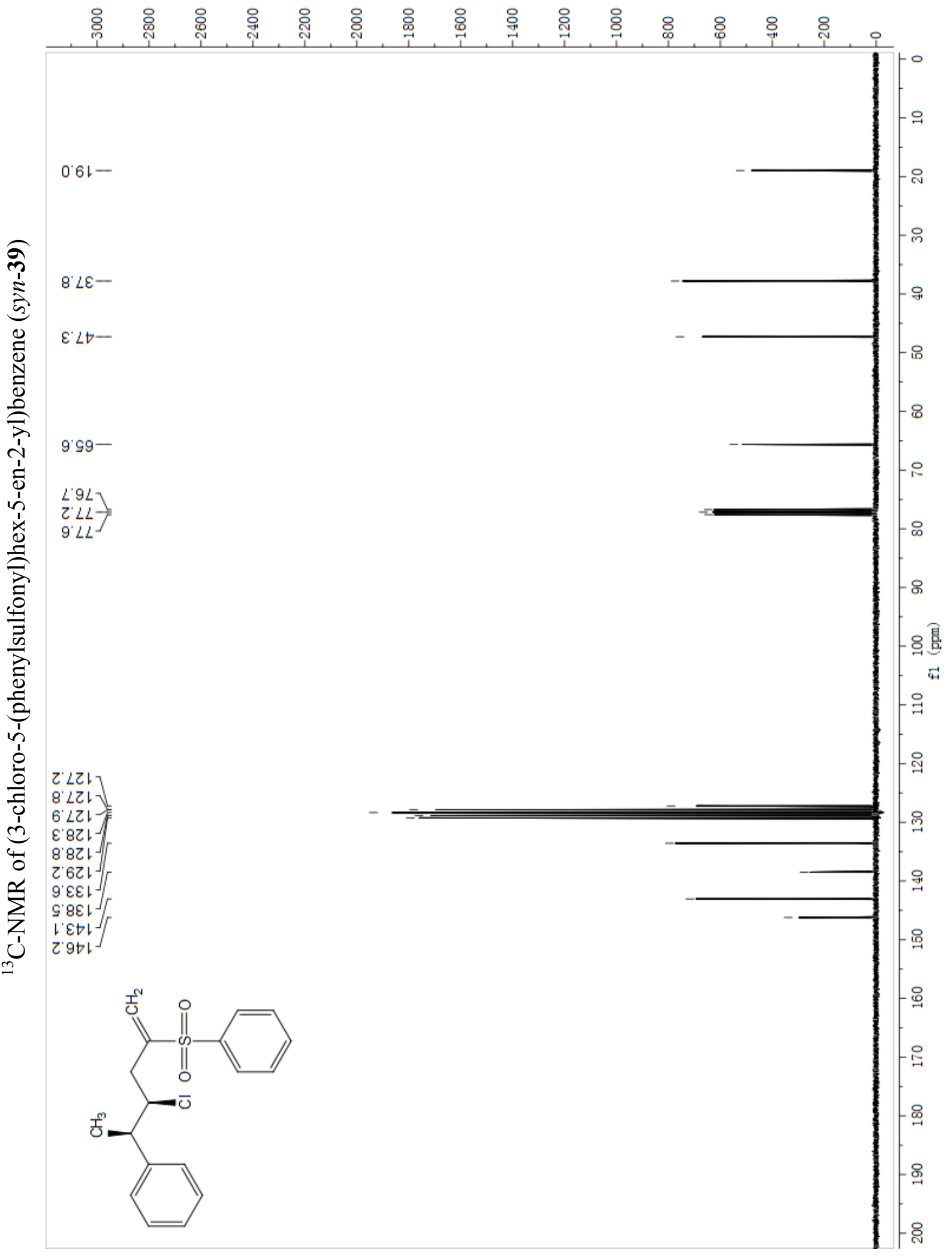




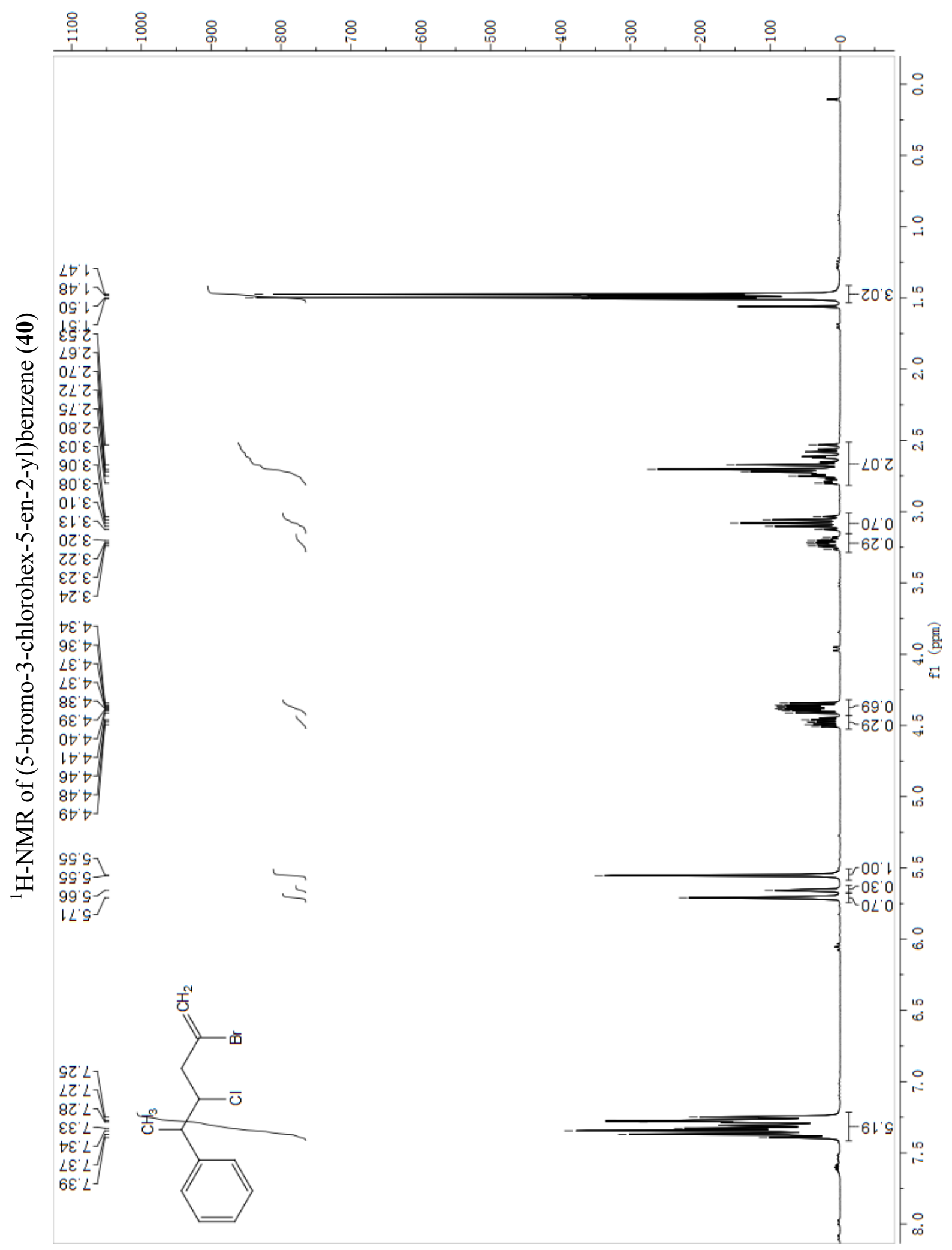




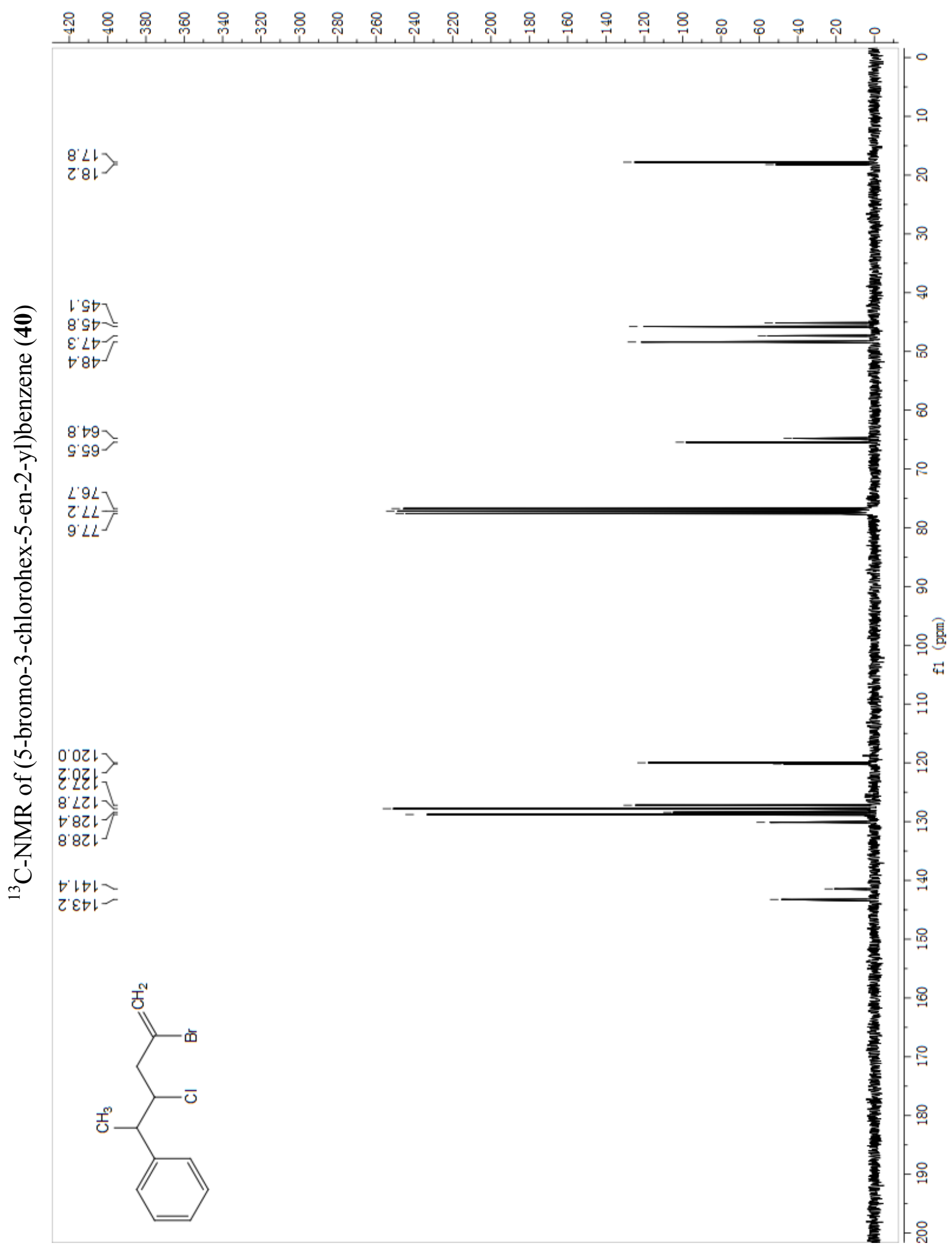




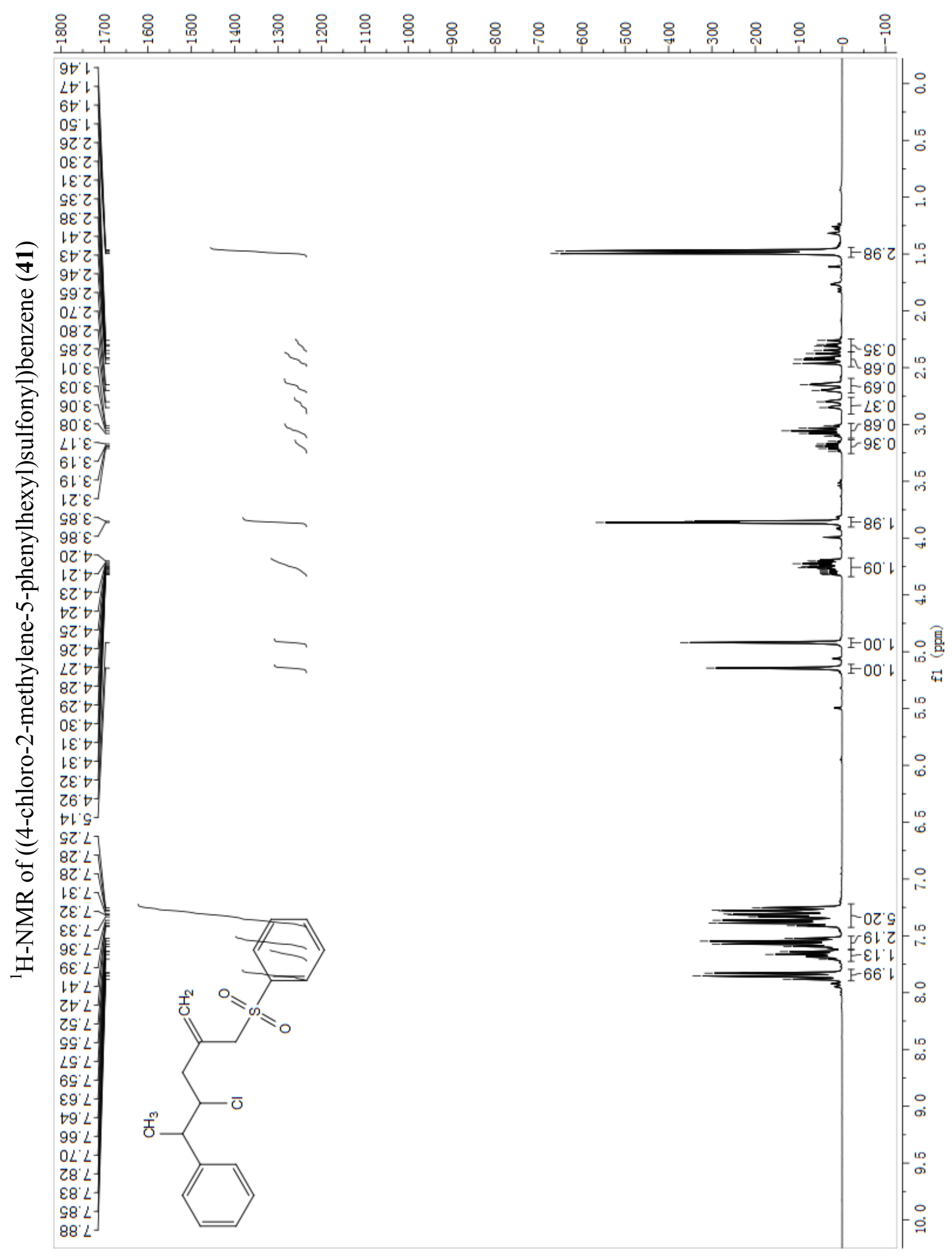




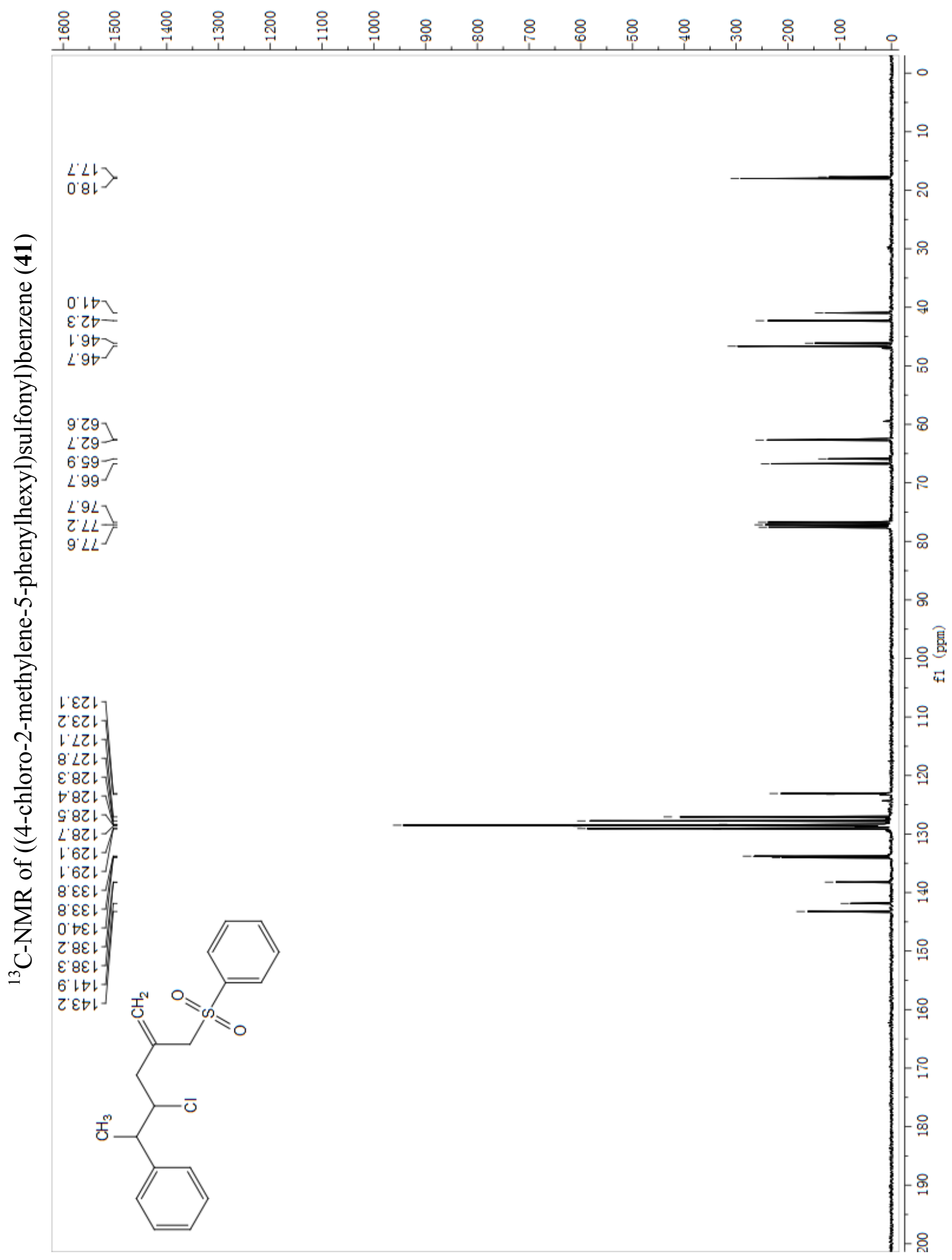




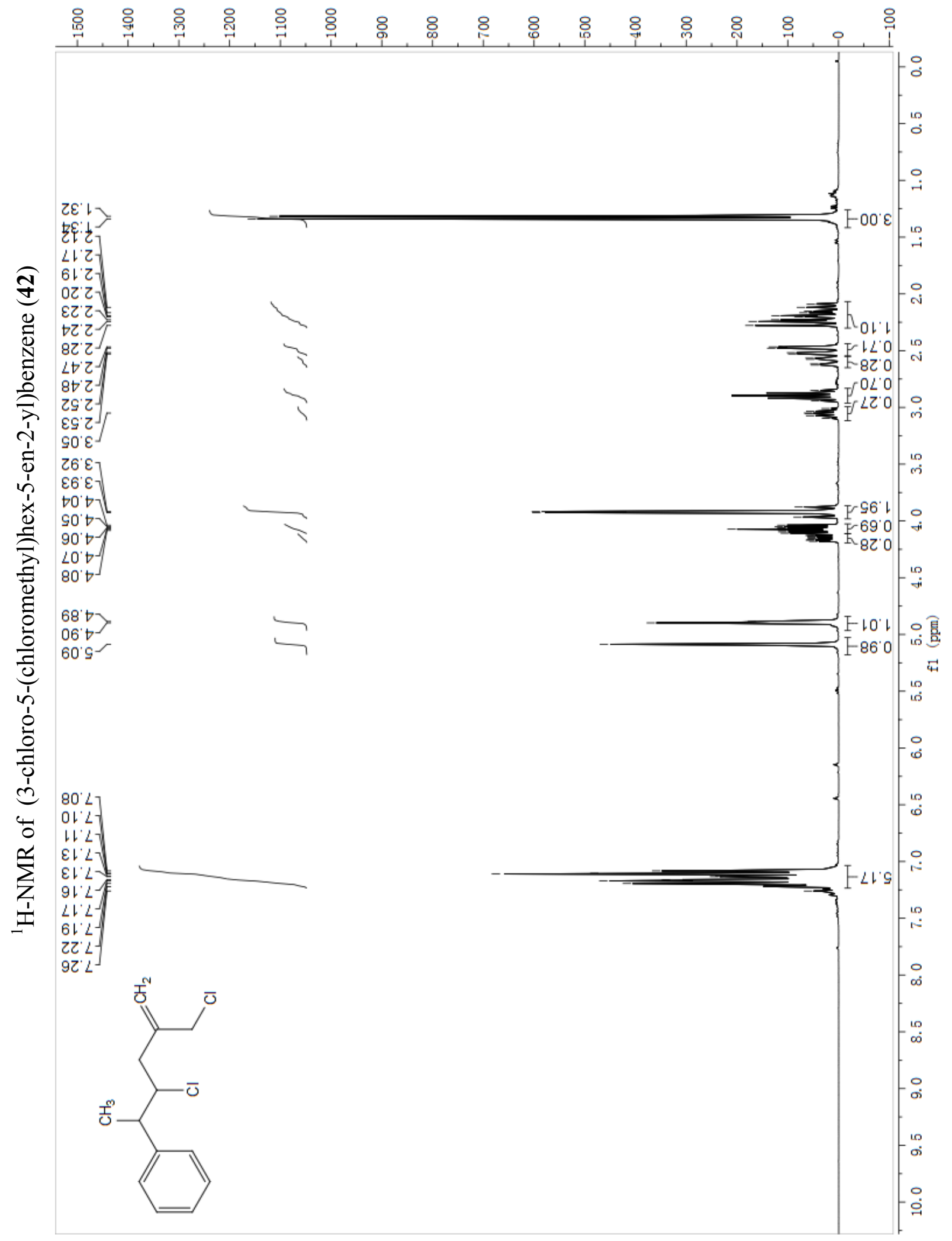




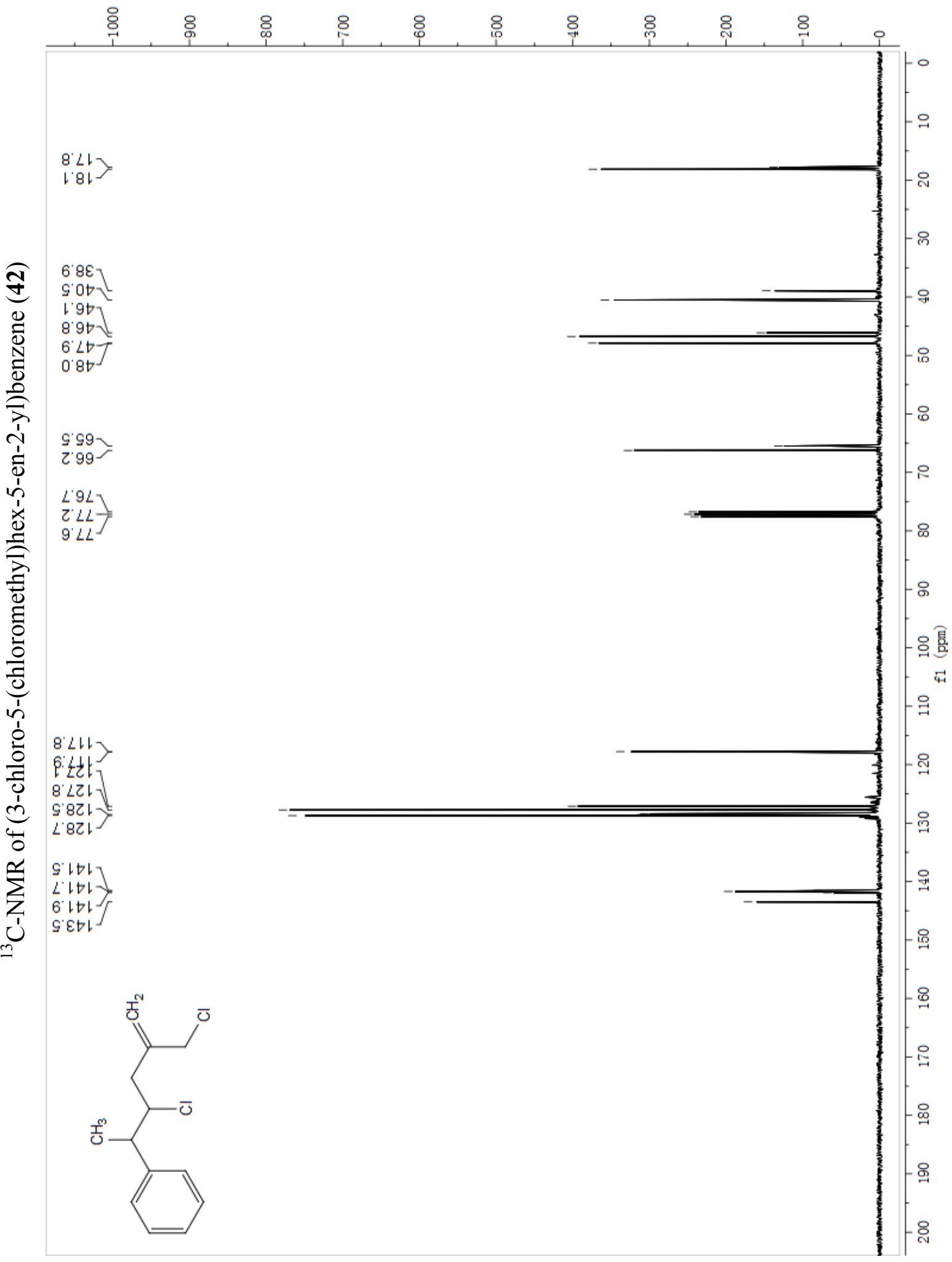




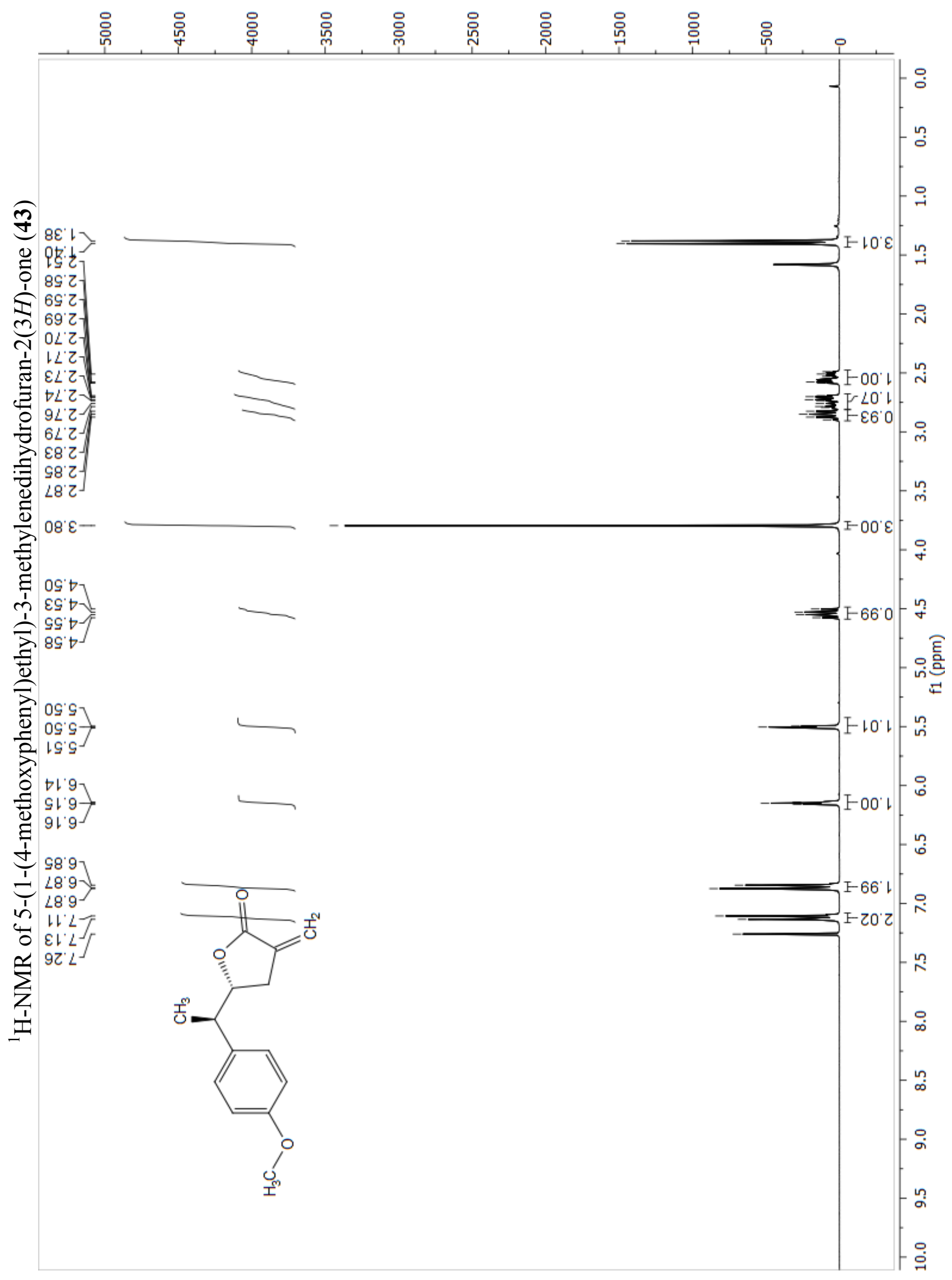




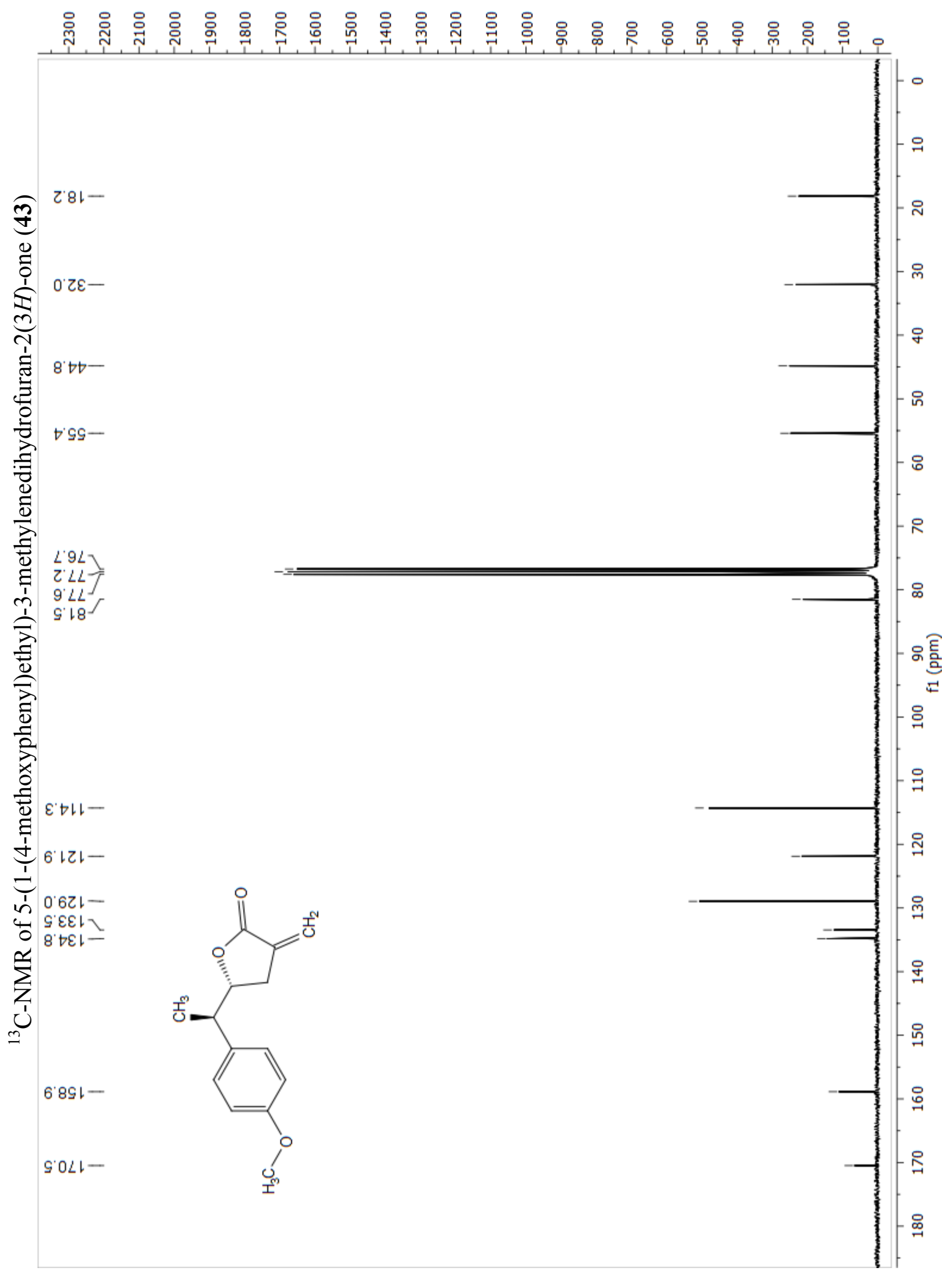




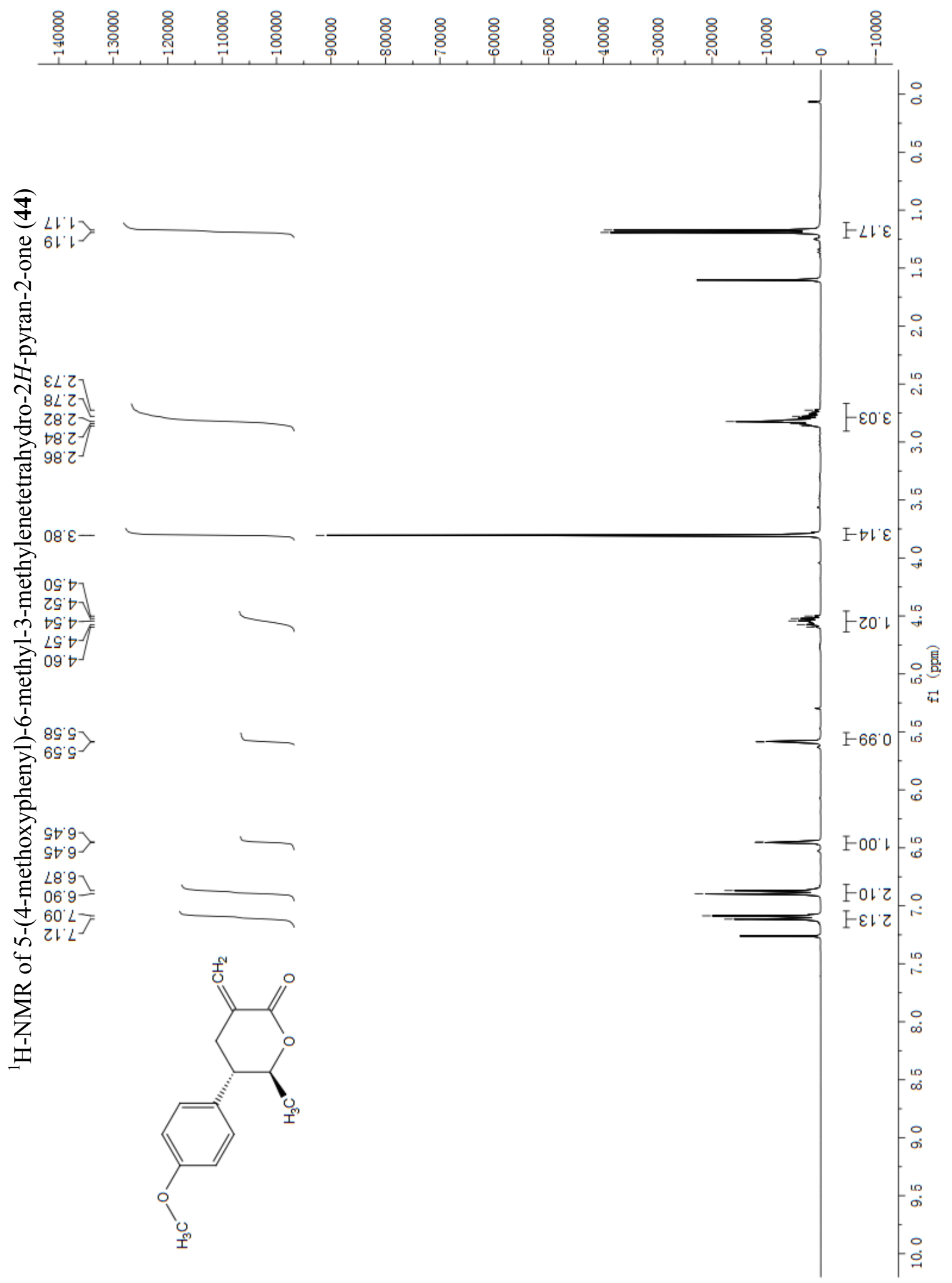




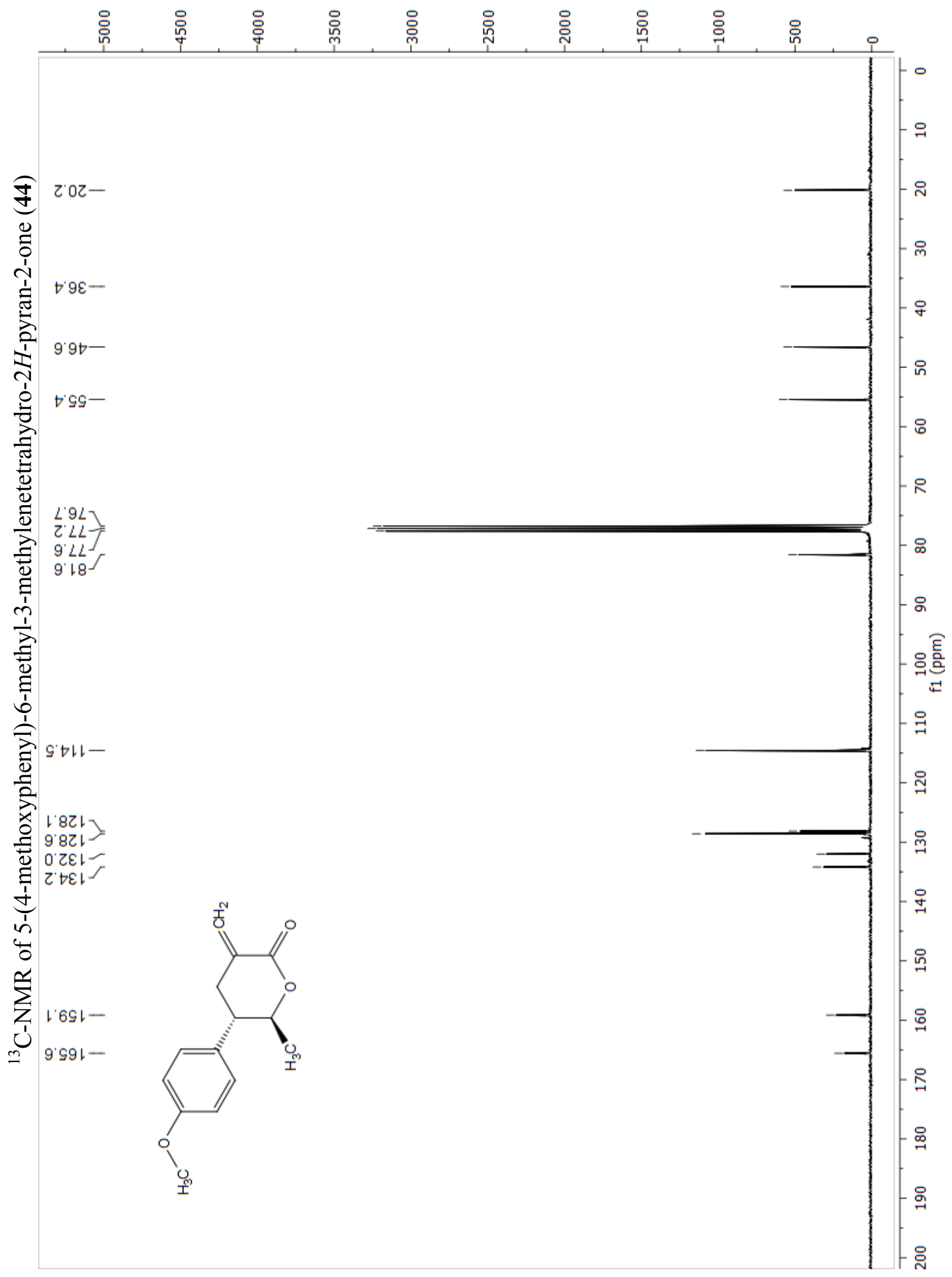




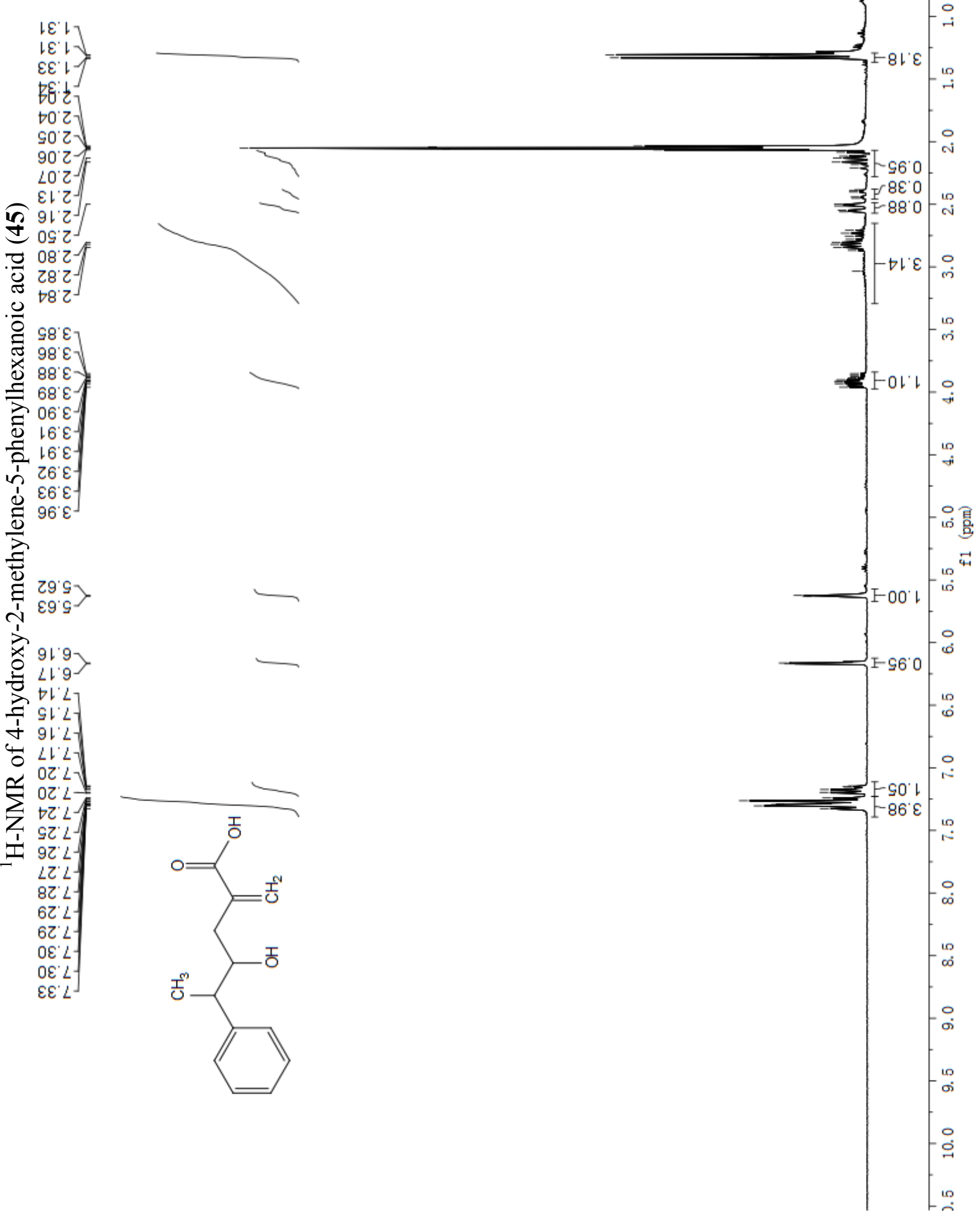




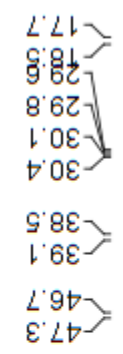

8

$\frac{\pi}{0}$

일

$\stackrel{x}{2}$

过
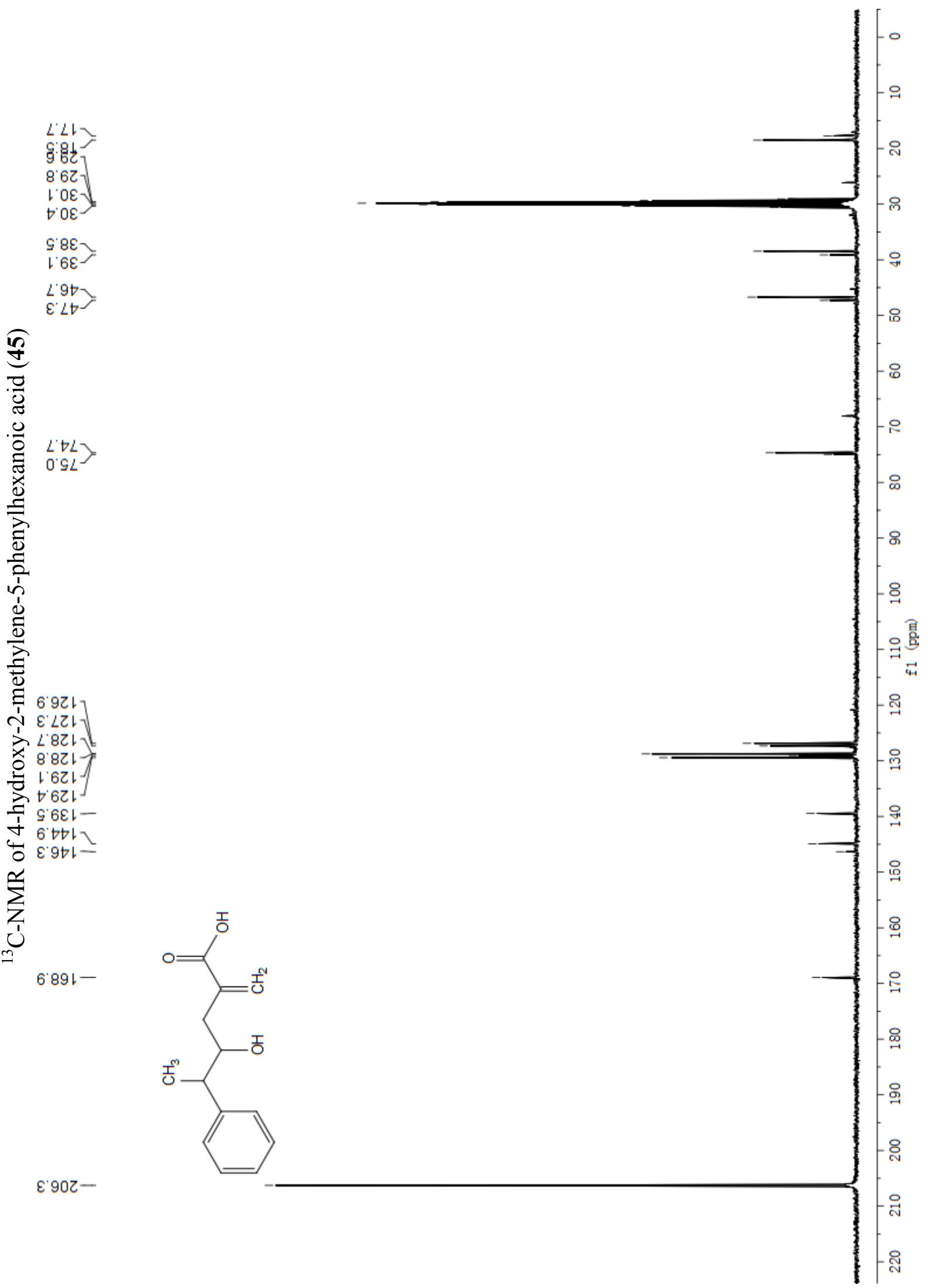

$\varepsilon 902-$

$\varepsilon^{\prime} \angle Z L$

ᄀ $\angle 821$

8821

는 62

入 $\nabla^{\circ} 62$

G $6 \varepsilon 1-$

$\forall 6^{\prime} \nabla t \downarrow$

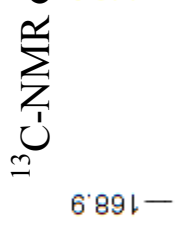



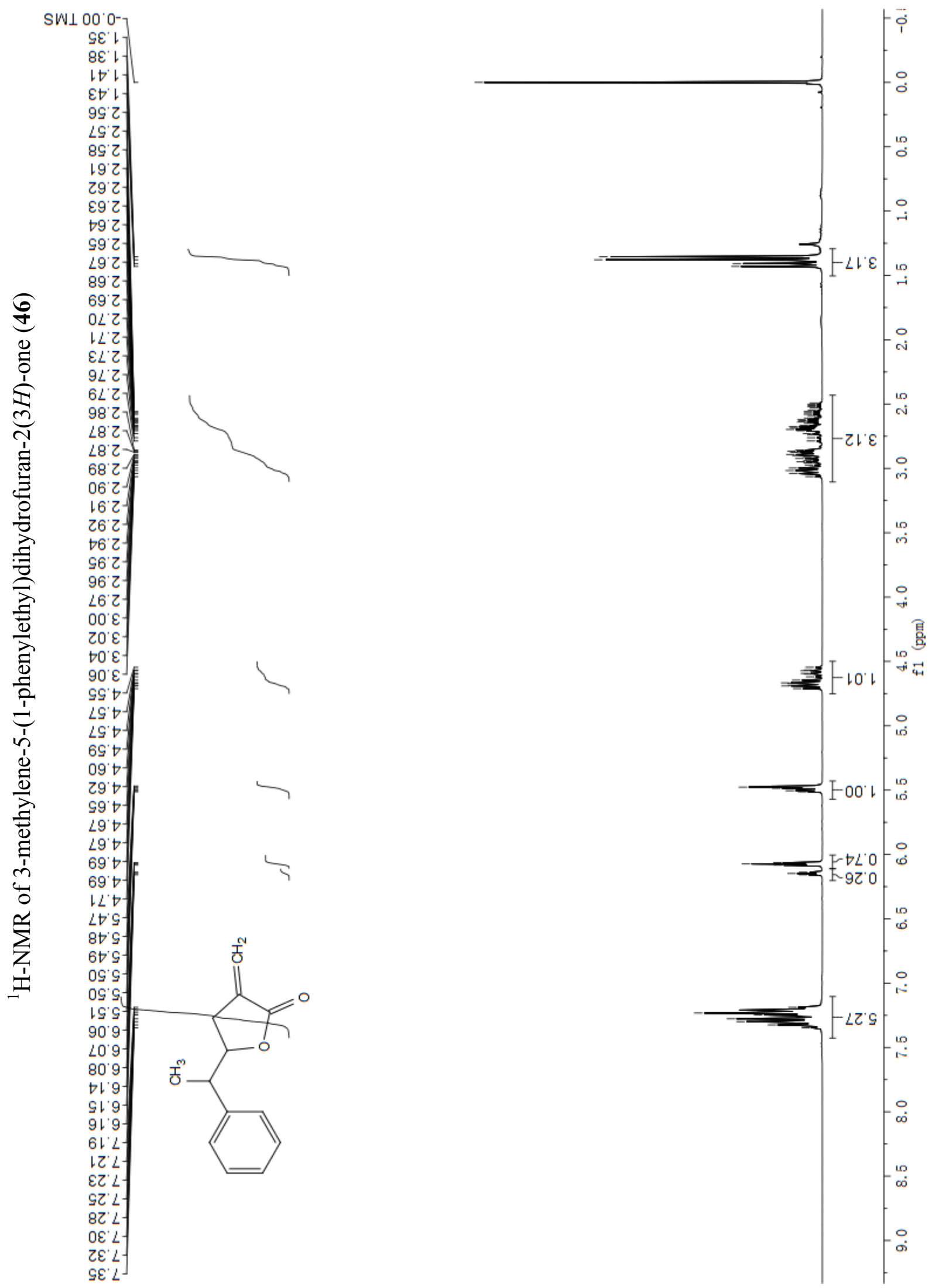
ย'91

0.81 -

$0.1 \varepsilon=$
L.

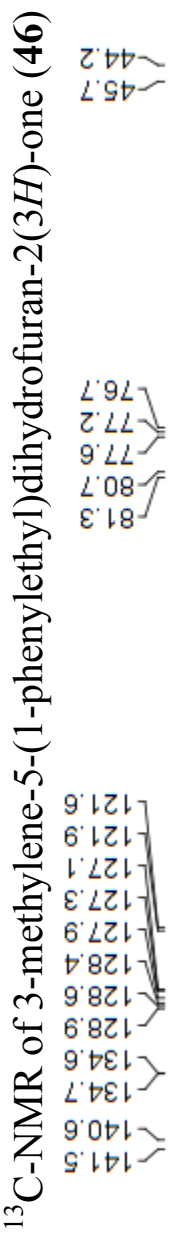

$\varepsilon 0<1>$

$\varepsilon 0<1$
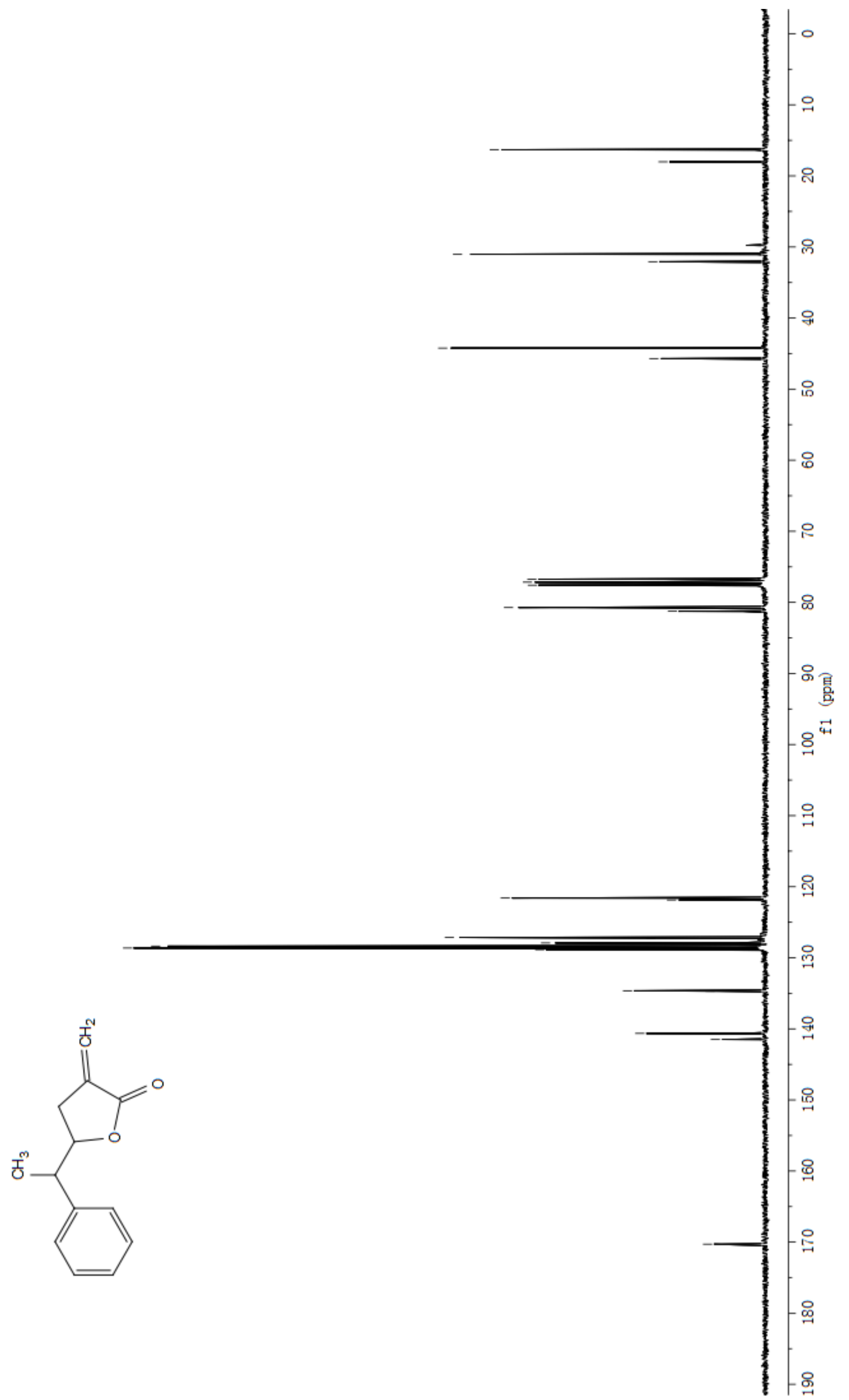
74

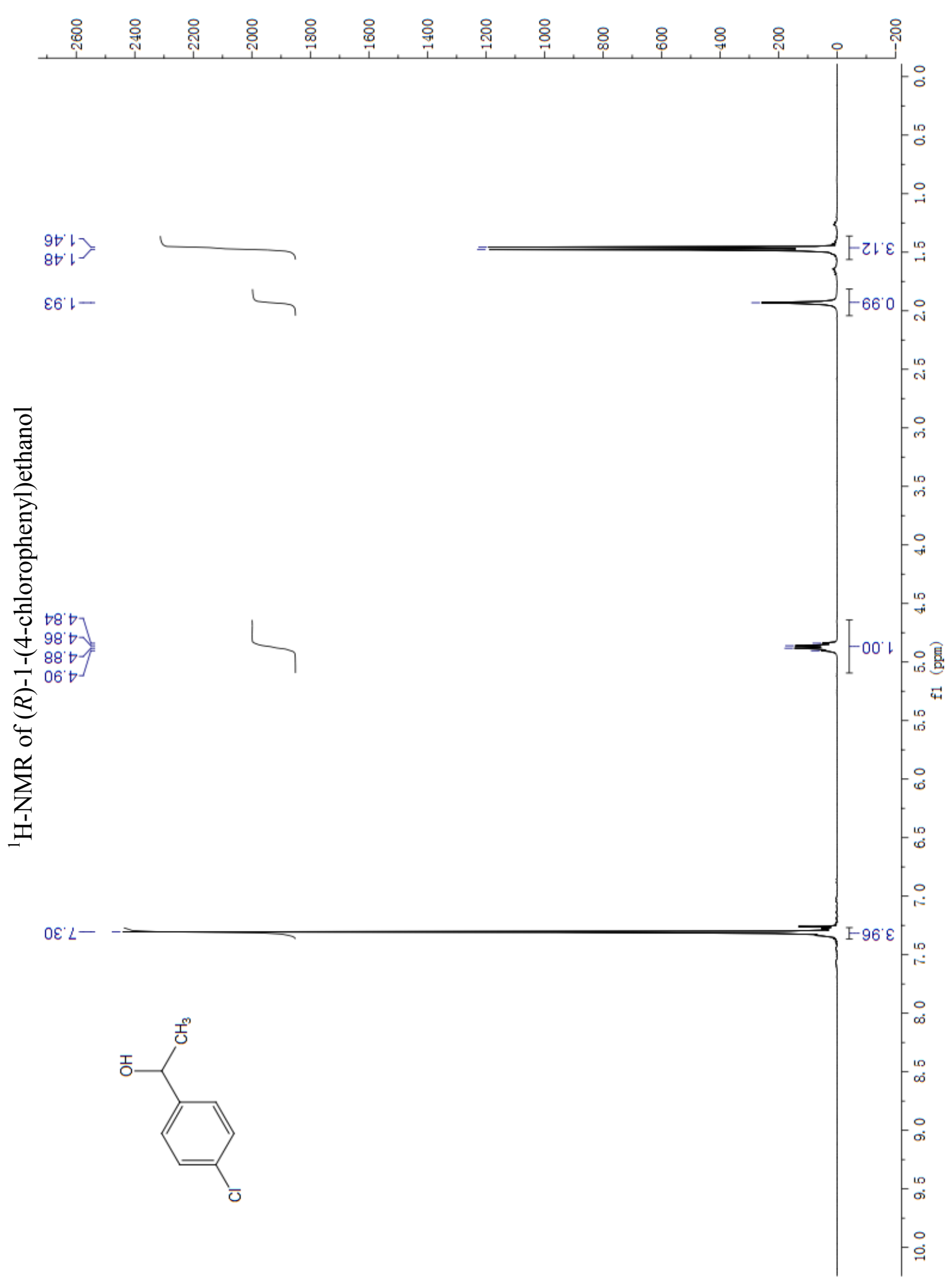




$$
4
$$




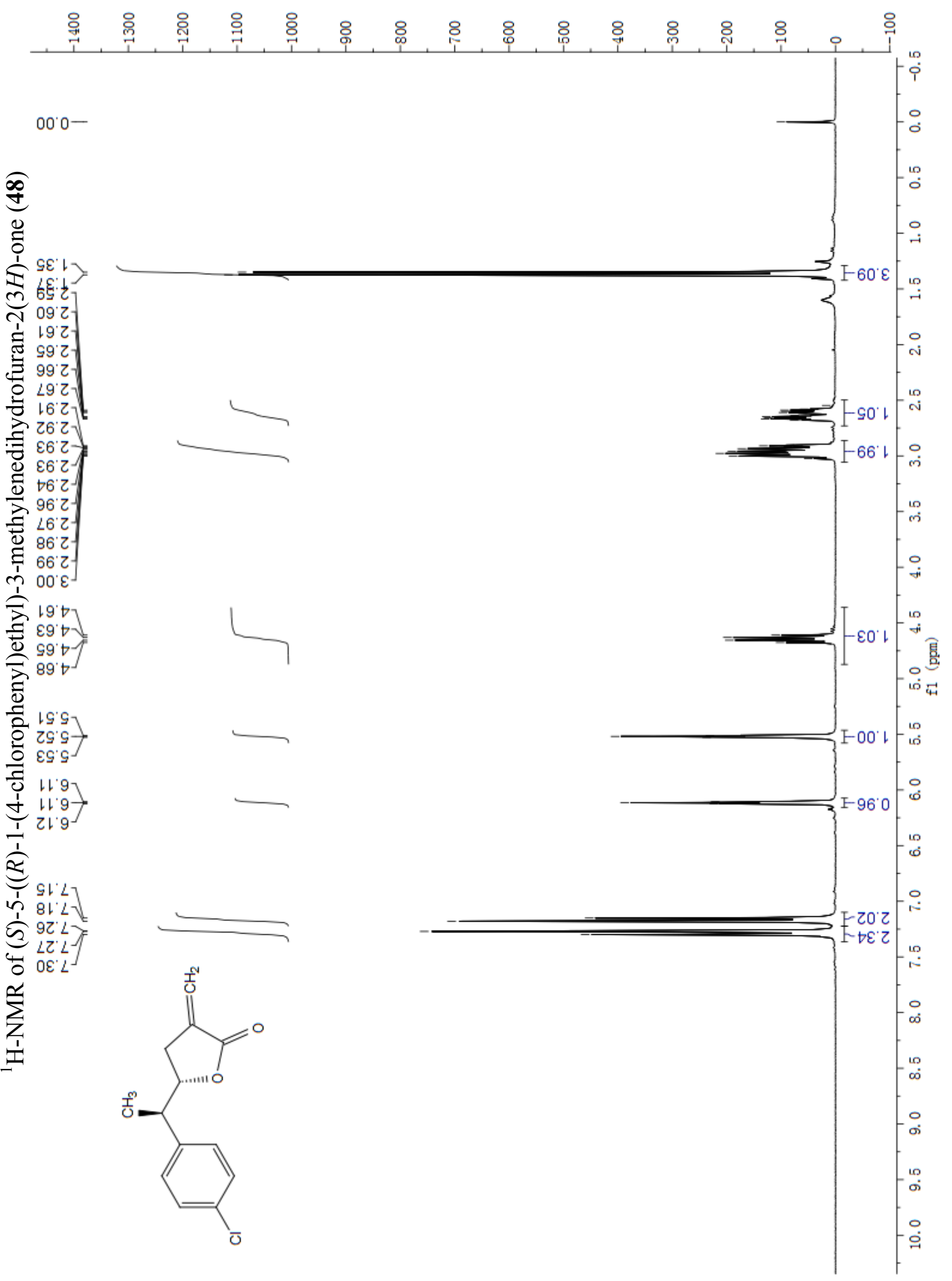


77

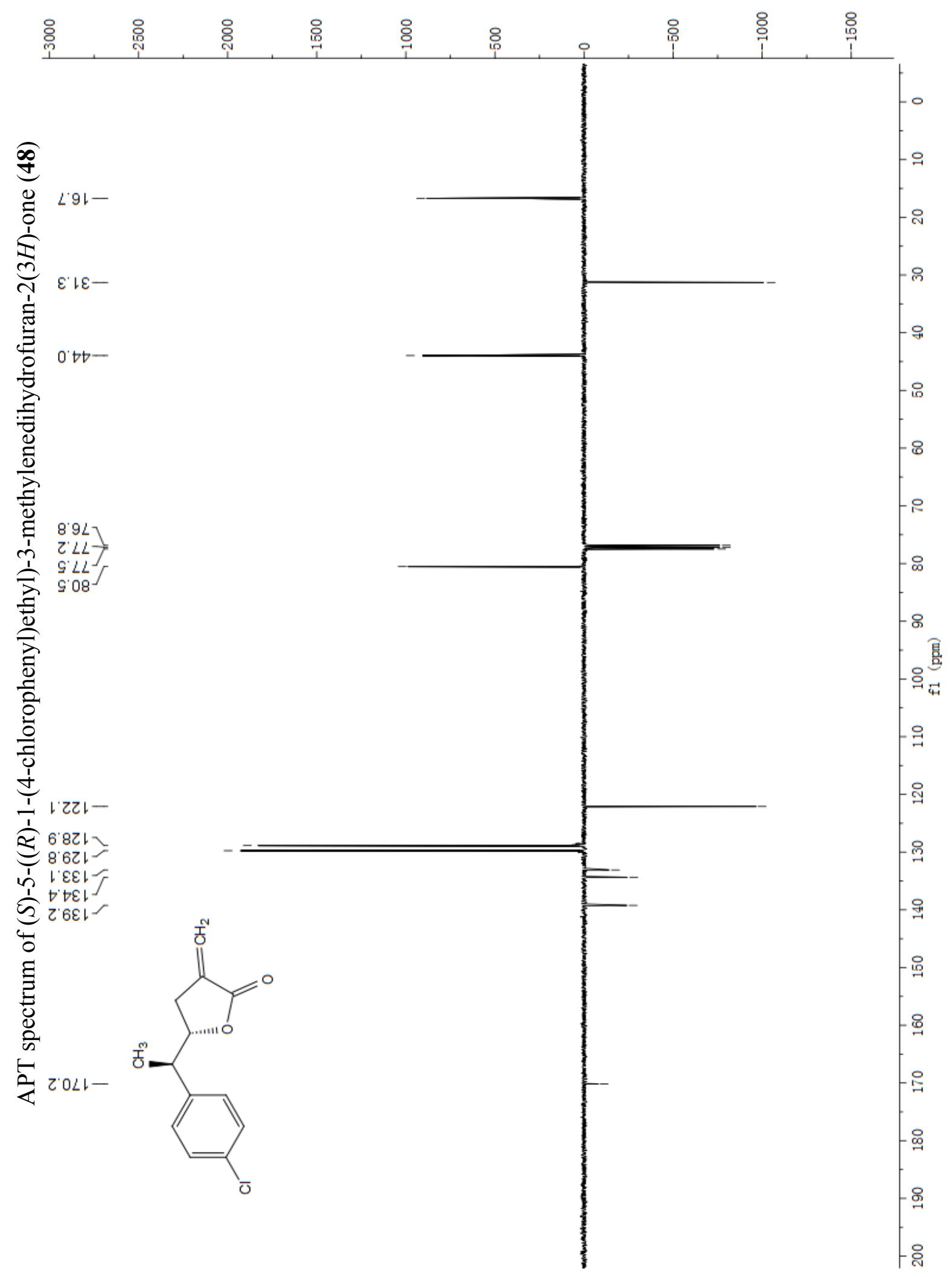




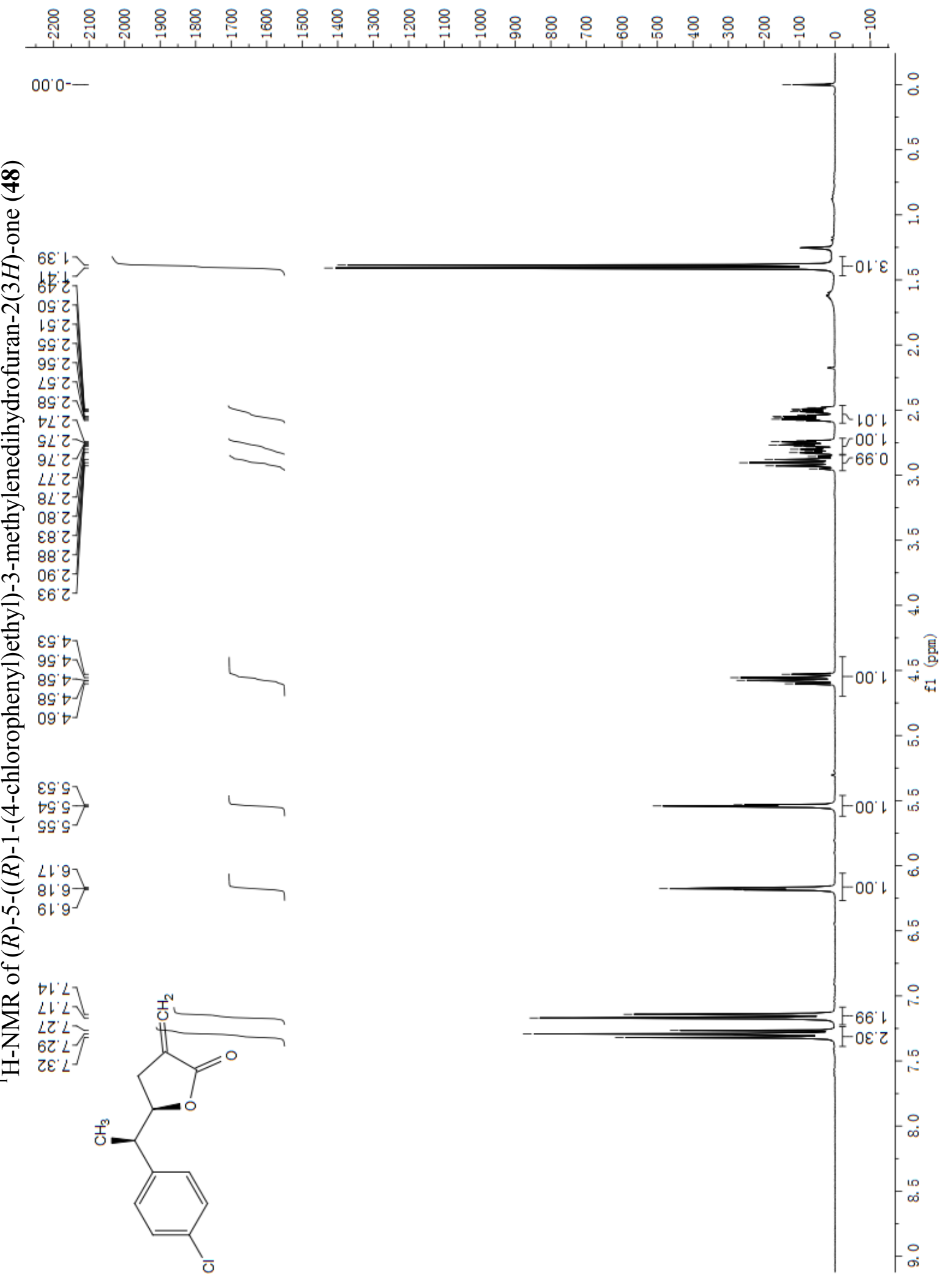




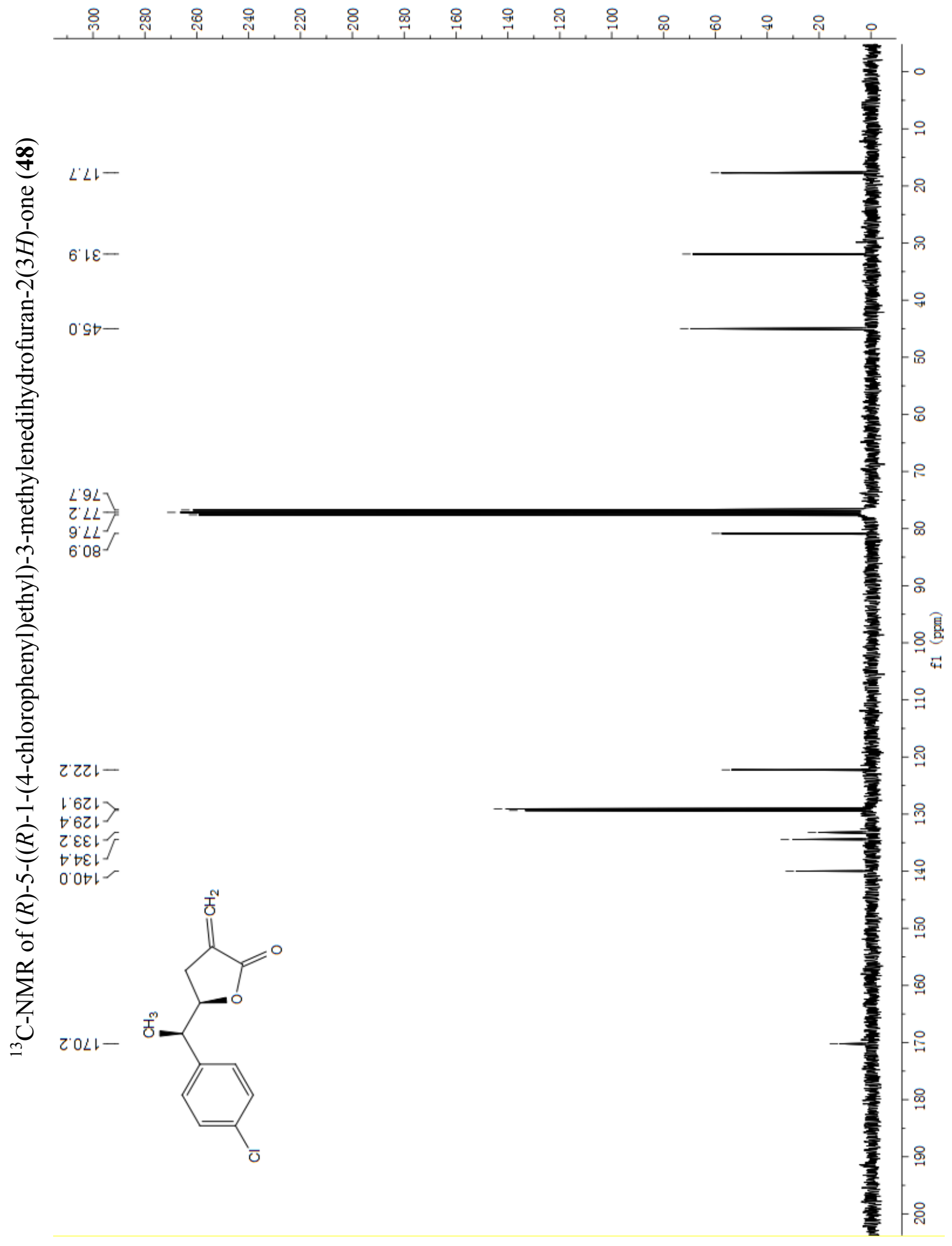




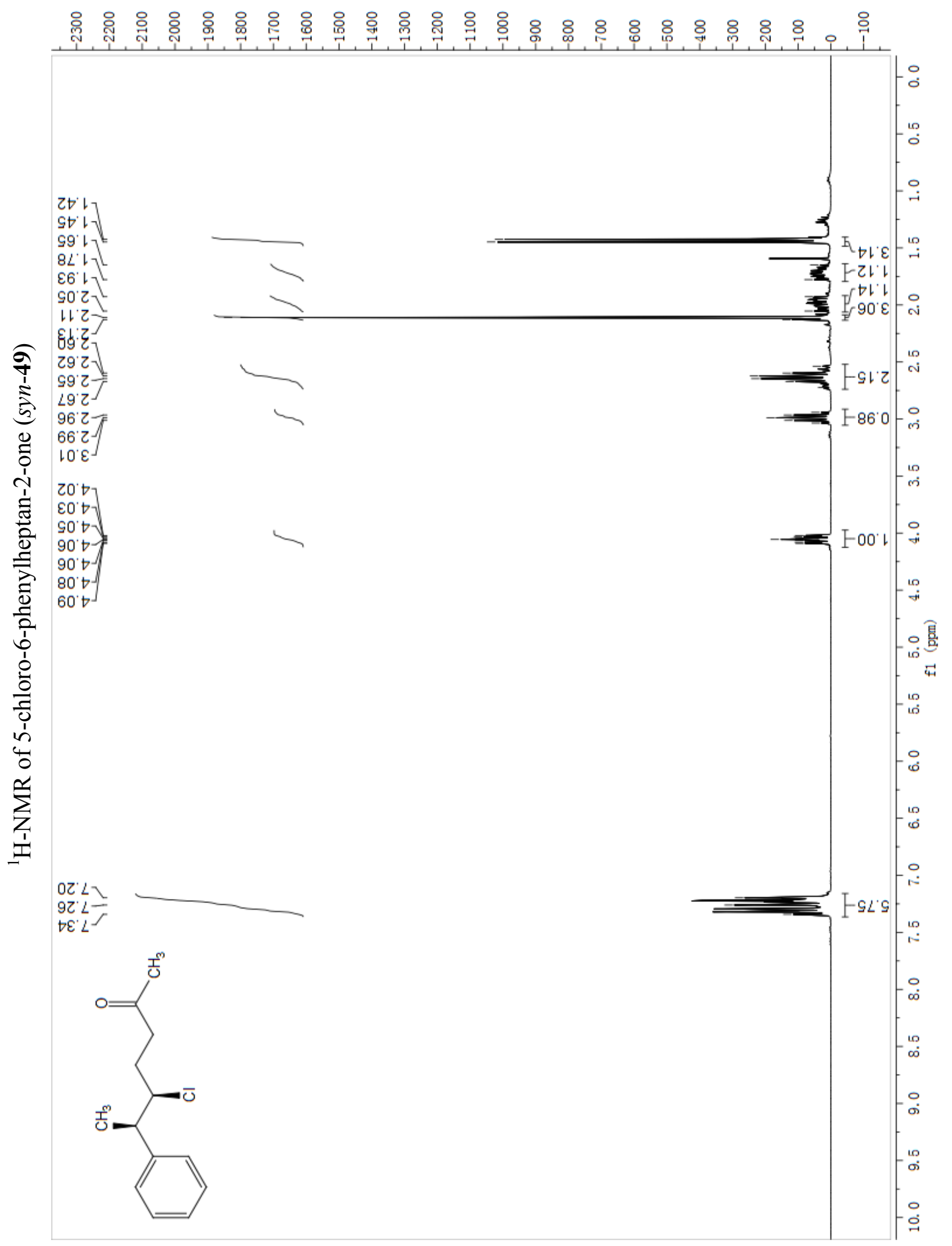




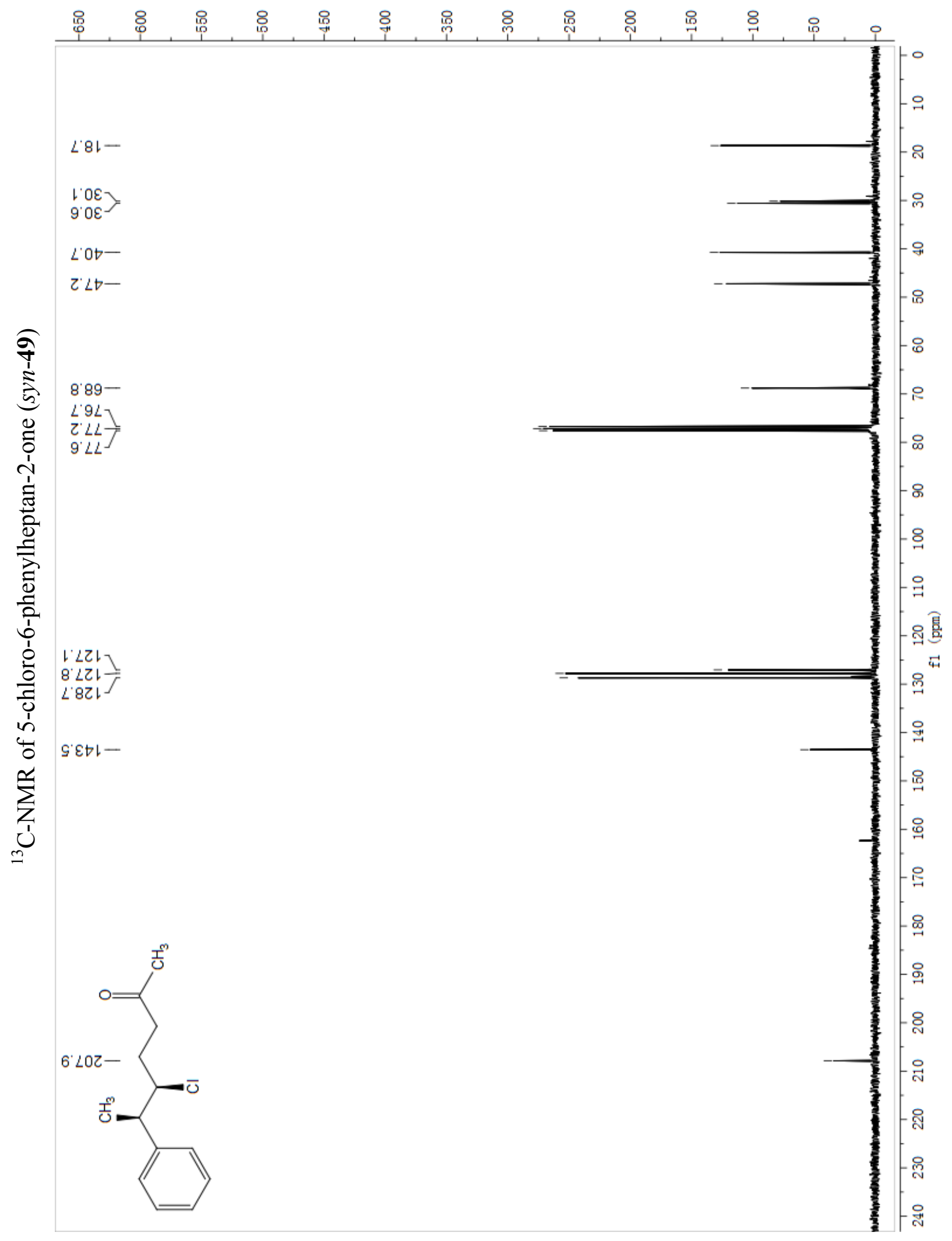




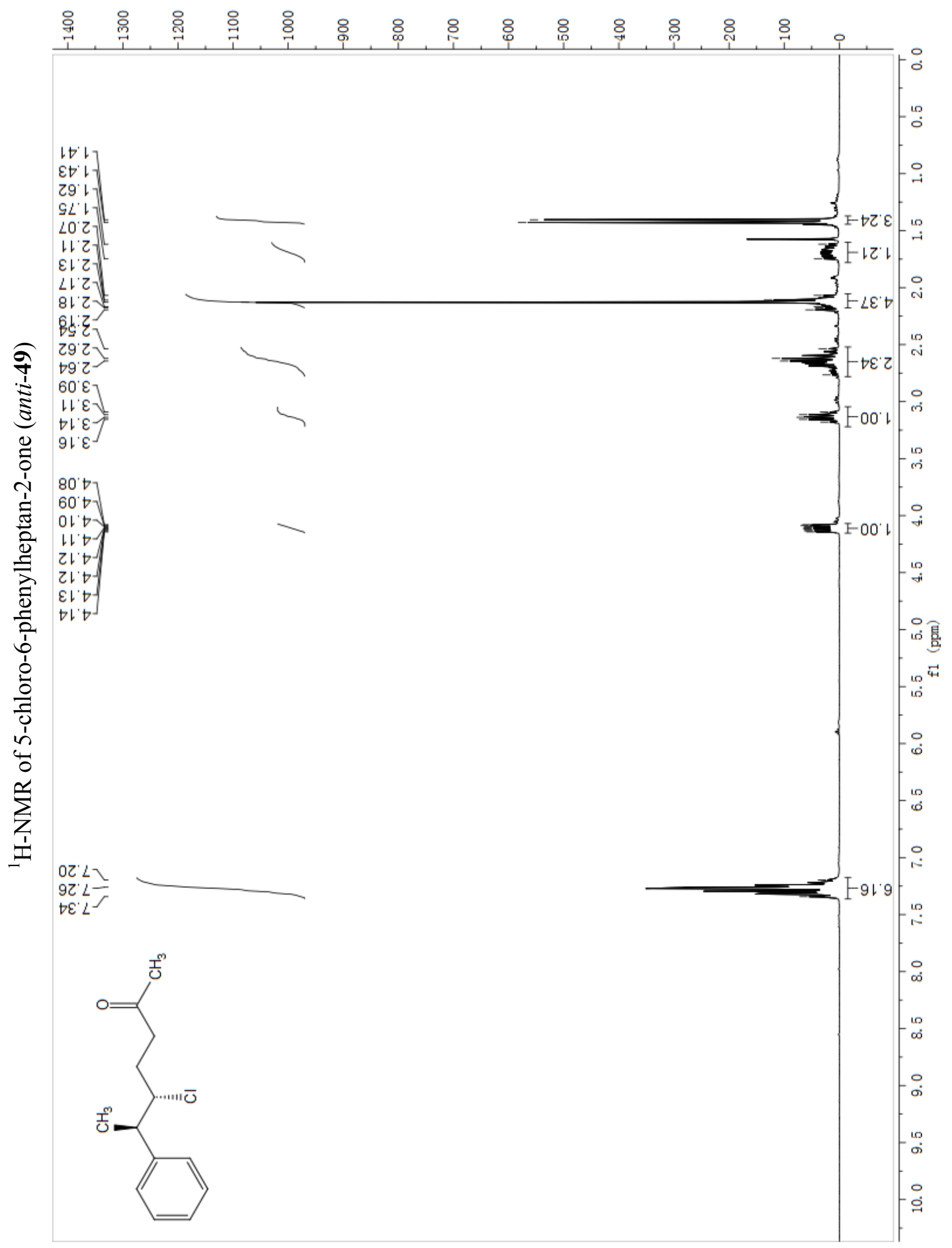


83

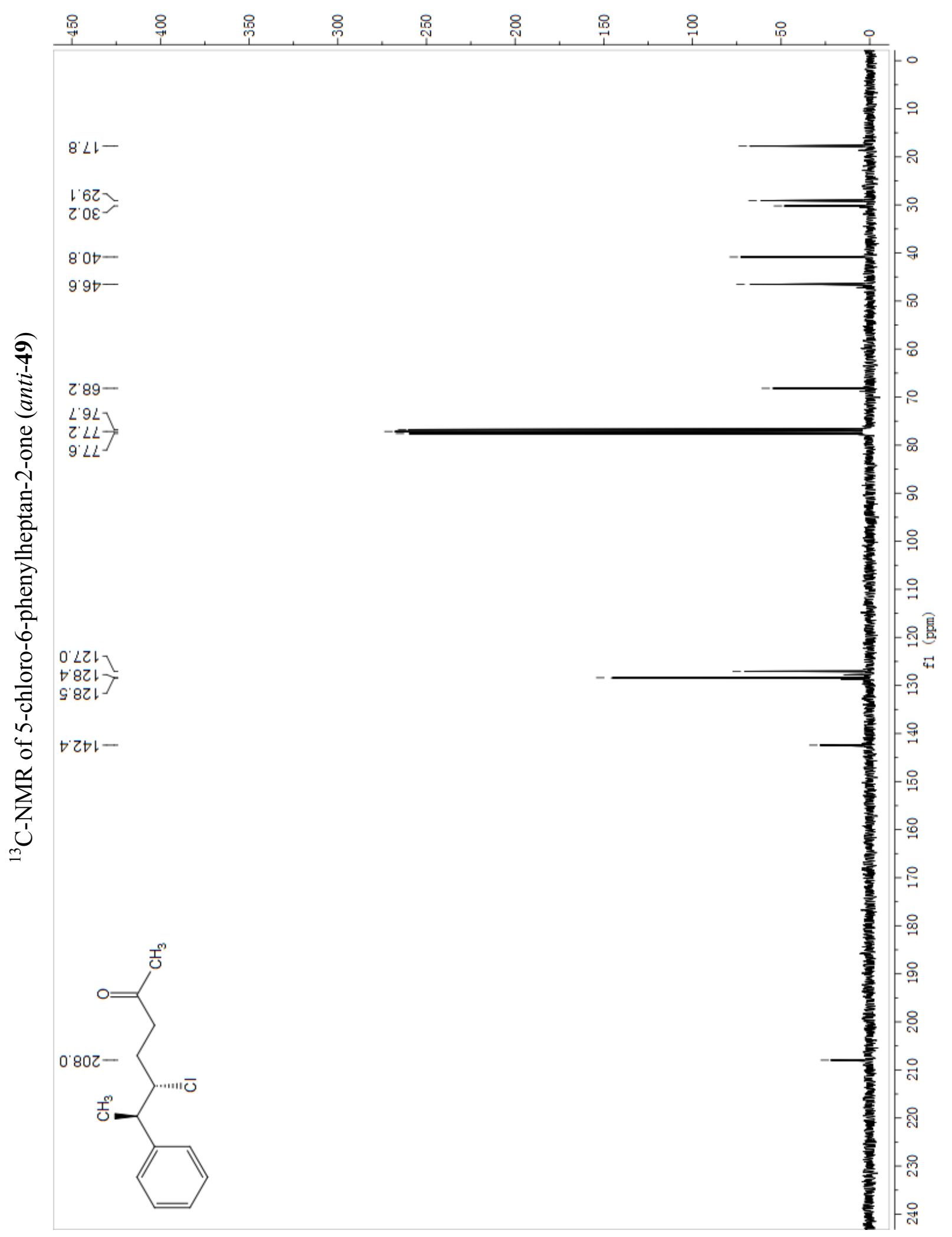




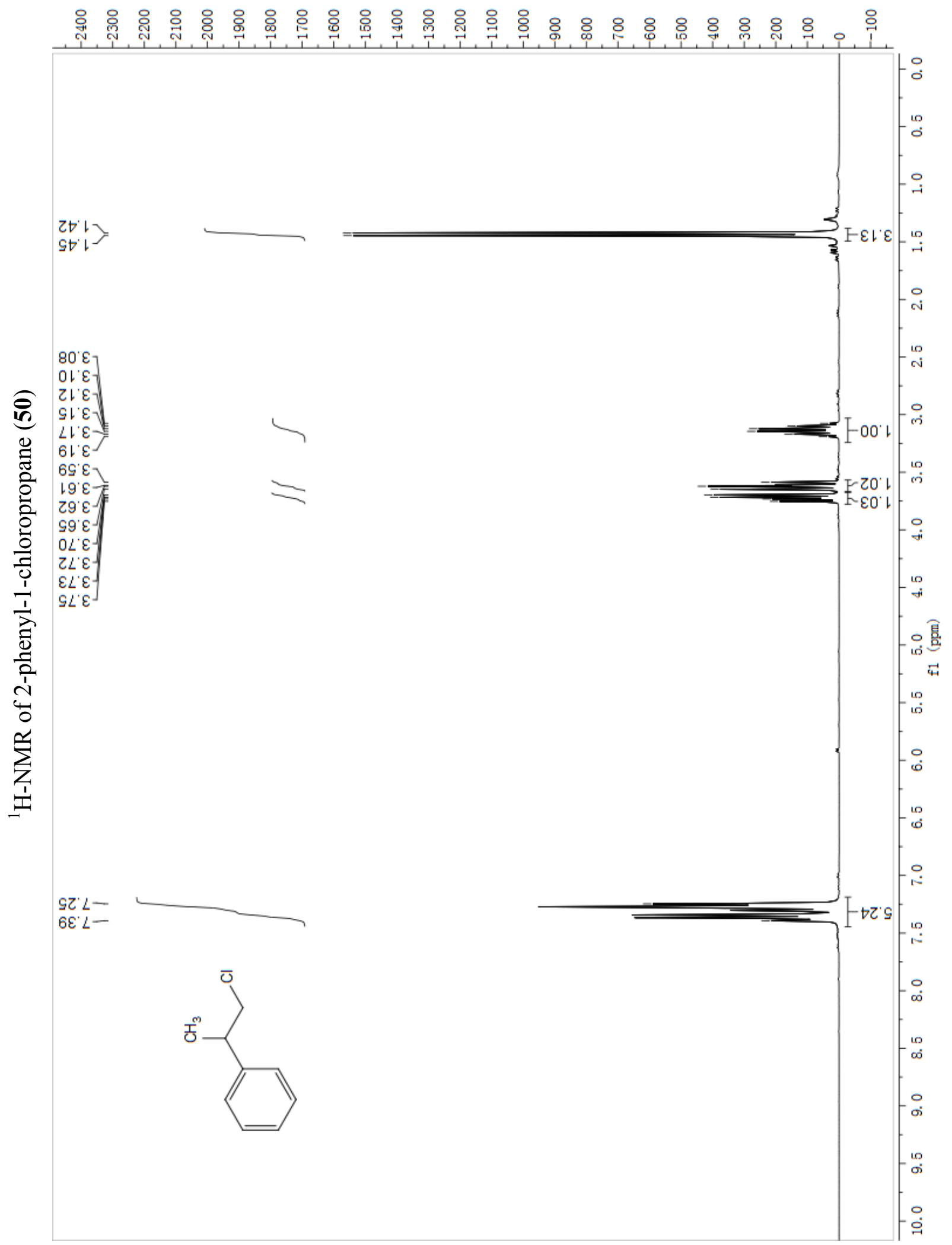




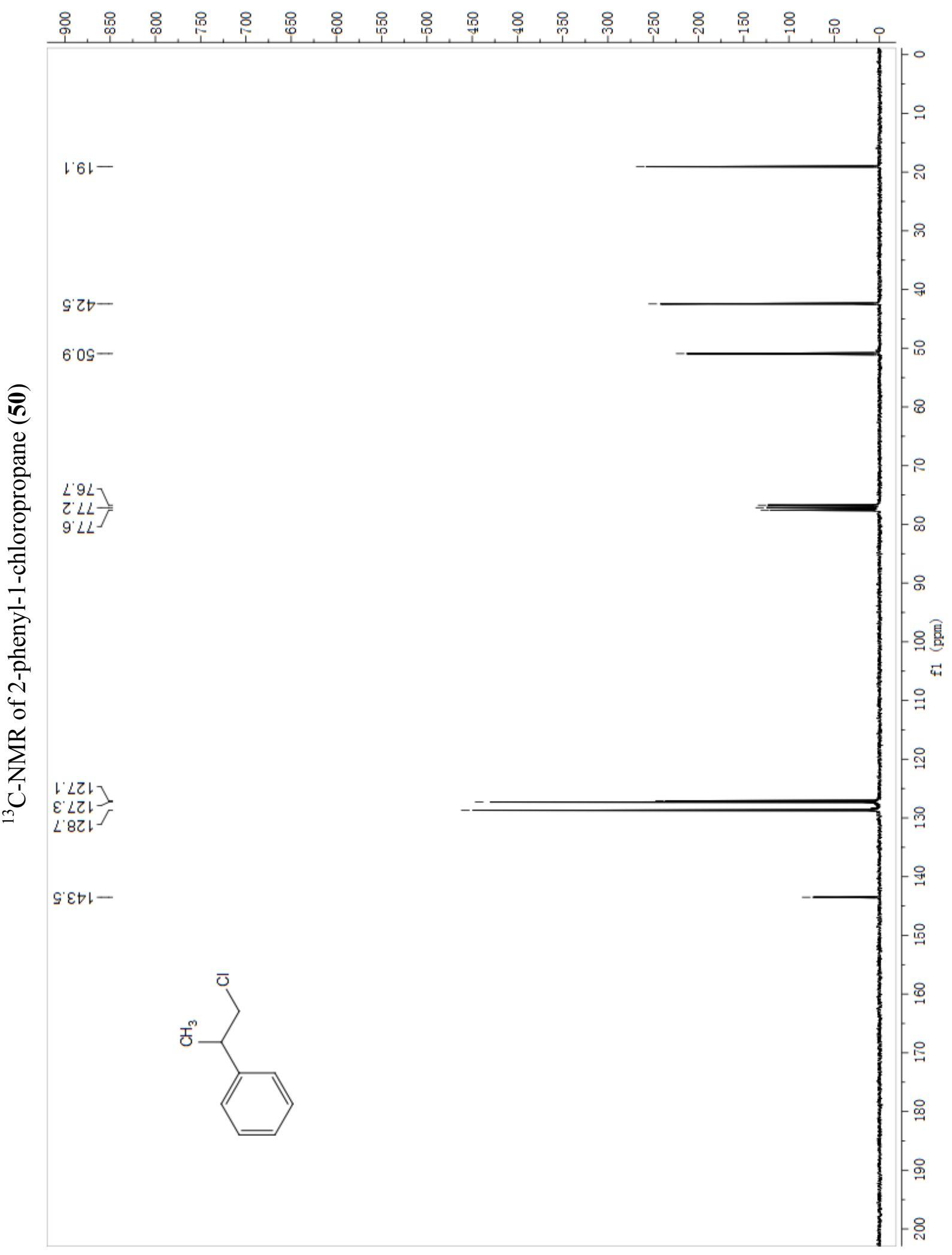




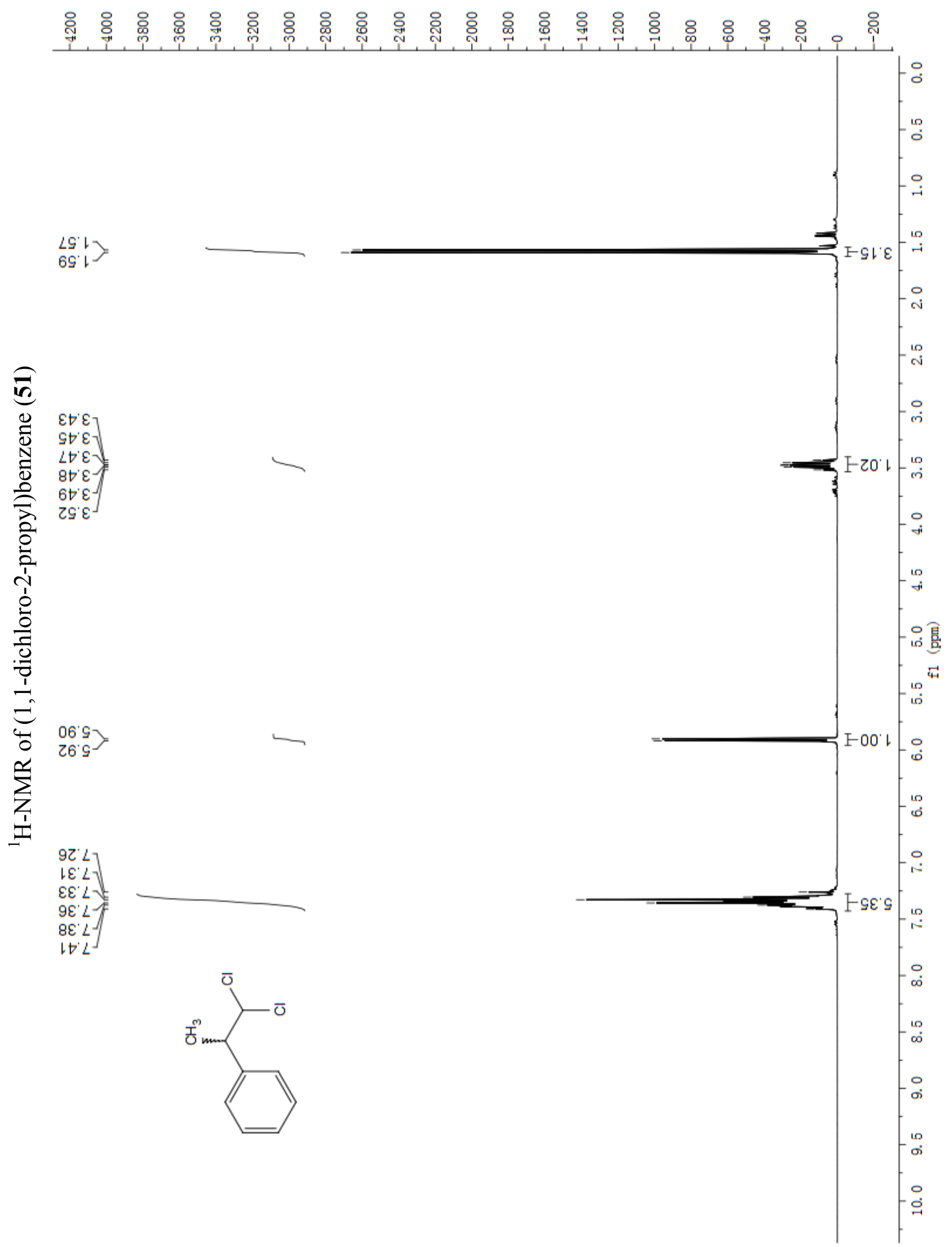




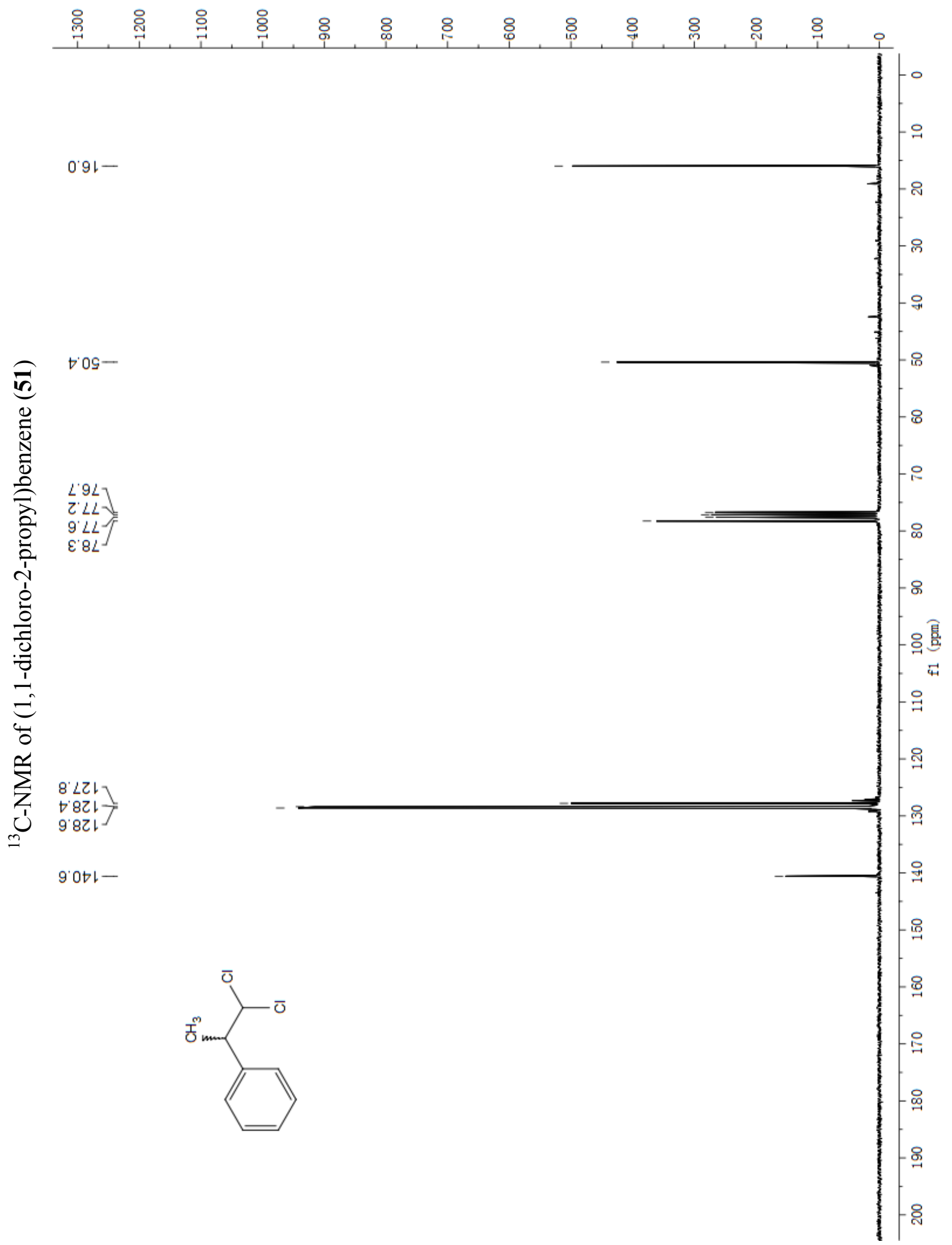




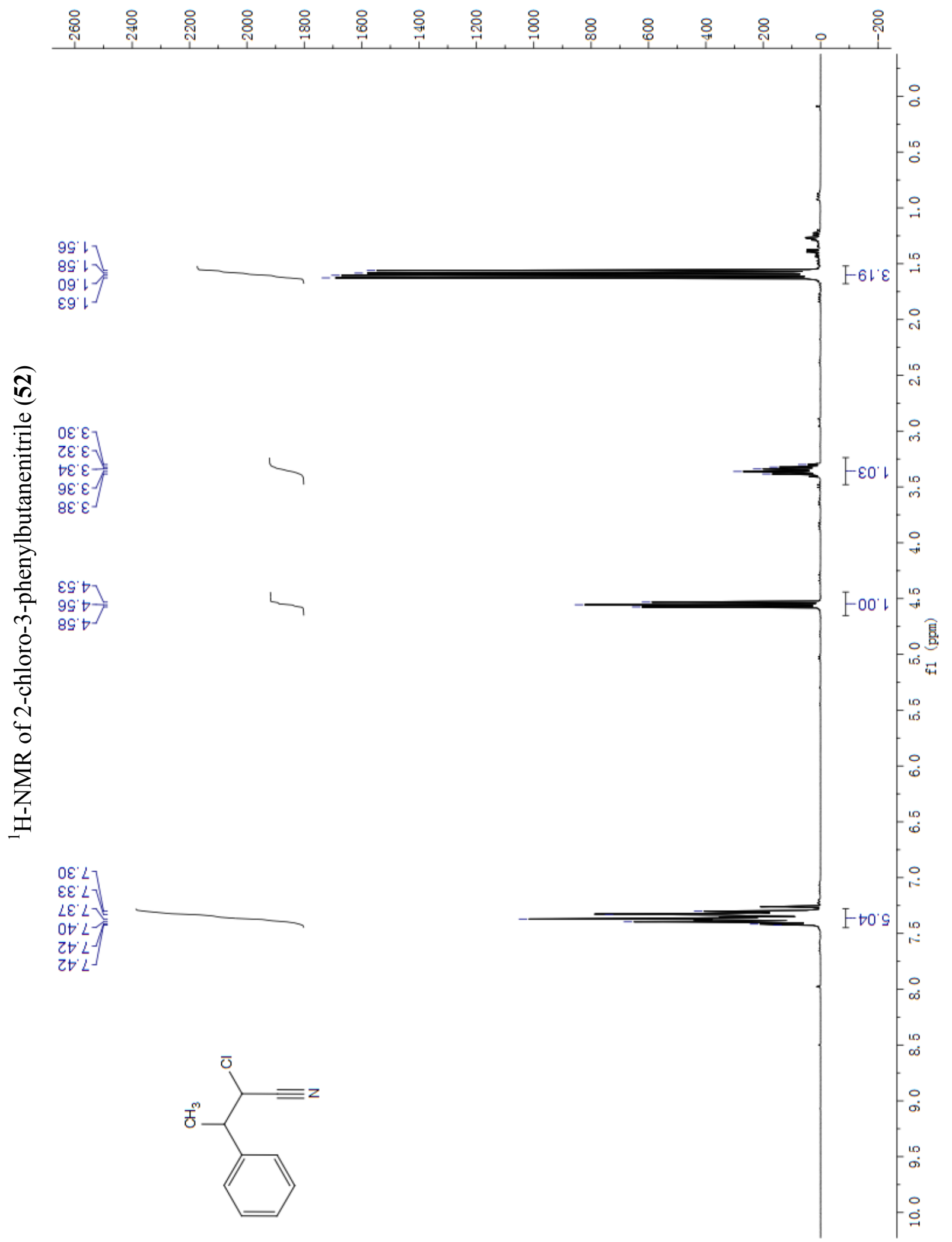




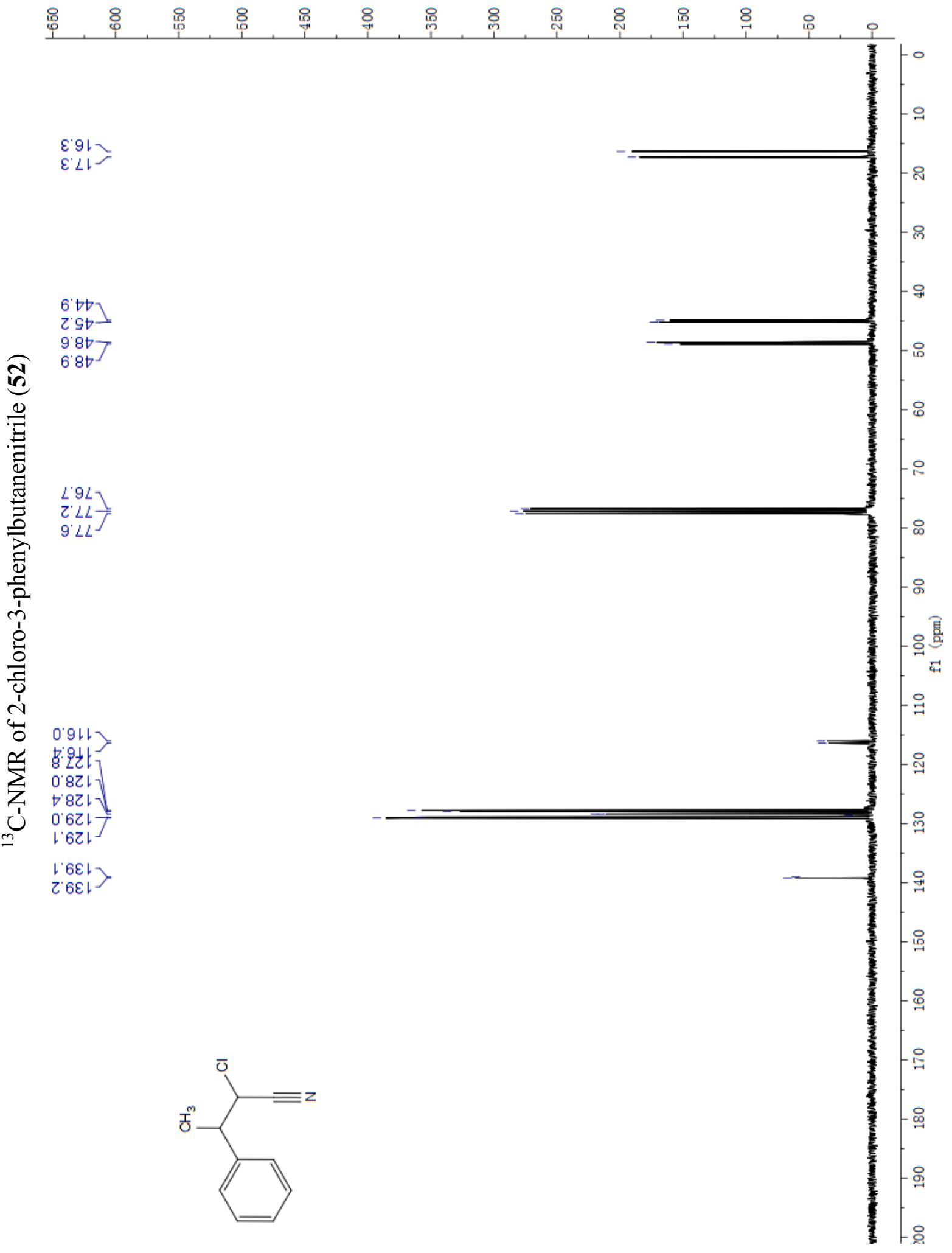




\section{(R)-1-(4-chlorophenyl)ethanol}

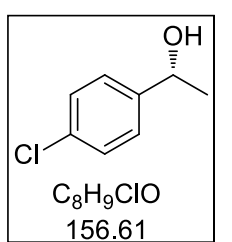

The enantiomeric purity of 1-(4-chlorophenyl)ethanol was determined to be $92.4 \%$ by GC analysis with a chiral stationary phase column in comparison with racemic material. $\mathrm{RT}=203.9 \mathrm{~min}(R$ isomer $), 230.8 \mathrm{~min}$ ( $S$ isomer $)$; GC-type: Trace GC Ultra; Column type: $\quad$ FS $32025, \quad 50 \%$ 6-TB-2.3 di gly $\beta$ in PS 086, df: $0.25 \mu \mathrm{m}$, length: $20 \mathrm{~m}, 50 \mathrm{kPa}$, constant flow: 1.8 $\mathrm{mL} / \mathrm{min}$, helium; Oven Method: Initial temperature: $80{ }^{\circ} \mathrm{C}$, Initial time: $240 \mathrm{~min}$, Number of ramps: 1 , rate: $4{ }^{\circ} \mathrm{C} / \mathrm{min}$, final temperature: $210^{\circ} \mathrm{C}$, hold time: $20 \mathrm{~min}$.

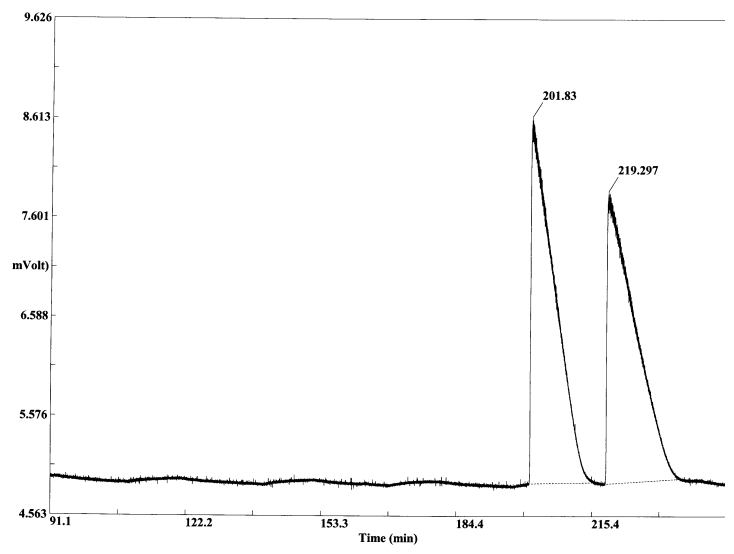

Peak \# Area \% Ret. Time

$1 \quad 50.5817 \quad 201.834$

$\begin{array}{lll}2 & 49.4183 & 219.304\end{array}$

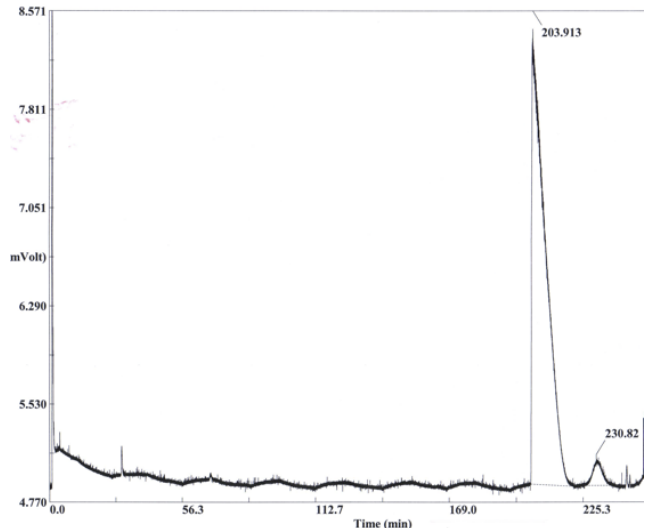

Peak \# Area \% Ret. Time

$\begin{array}{lll}1 & 96.1843 \quad 203.914\end{array}$

$\begin{array}{lll}2 & 3.8157 & 230.824\end{array}$ 


\section{(4R,5R)-Ethyl 4-chloro-5-(4-chlorophenyl)-2-methylenehexanoate (36)}

The enantiomeric purity of minor diastereoisomer was determined to be

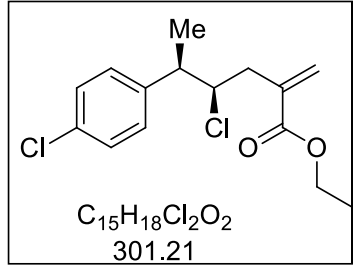
about $90 \%$ by GC analysis with a chiral stationary phase column in comparison with racemic material. GC analysis of crude product: $\mathrm{RT}=522.5$ min (major diastereoisomer), 599.9 min (minor diastereoisomer), $626.1 \mathrm{~min}$ (minor diastereoisomer). GC-type: Trace GC Ultra; Column type: FS 32025, 50\% 6-TB-2.3 di gly $\beta$ in PS 086, df: $0.25 \mu \mathrm{m}$, length: $20 \mathrm{~m}, 50 \mathrm{kPa}$, constant flow: $1.8 \mathrm{~mL} / \mathrm{min}$, helium; Oven Method: Initial temperature: $110^{\circ} \mathrm{C}$, Initial time: $700 \mathrm{~min}$, Number of ramps: 1 , rate: $6^{\circ} \mathrm{C} / \mathrm{min}$, final temperature: $210{ }^{\circ} \mathrm{C}$, hold time: $20 \mathrm{~min} .1$
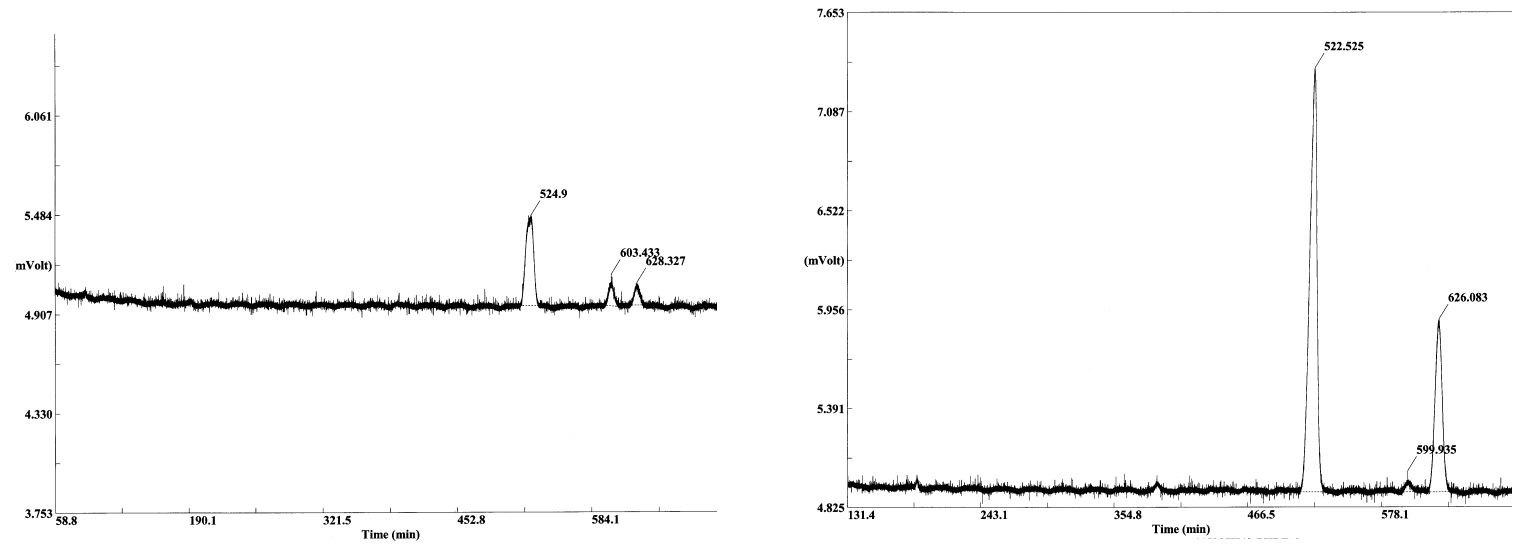

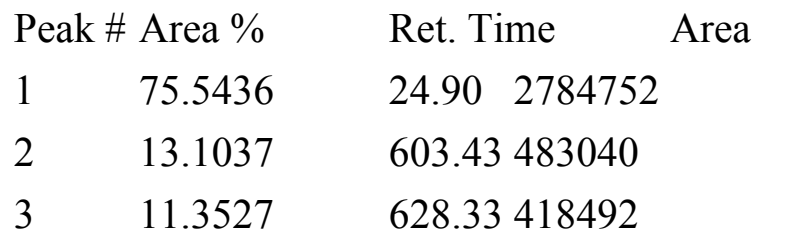

Peak \# Area \% Ret. Time

$1 \quad 70.4054 \quad 522.53$

$2 \quad 1.4761 \quad 599.94$

$3 \quad 28.1186 \quad 626.08$ 


\section{(4-Chlorophenyl)ethyl)-3-methylenedihydro-furan-2(3H)-one (48)}

The enantiomeric purity of $(S)-5-((R)-1-(4-c h l o r o p h e n y l) e t h y l)-3-m e t h y l e n e d i h y d r o-f u r a n-2(3 H)$ one 48 was determined to be $92.7 \%$ by GC analysis with a chiral stationary phase column in comparison with racemic material. RT $=548.2 \mathrm{~min}$ (minor isomer), $568.6 \mathrm{~min}$ (major isomer); GCtype: Trace GC Ultra; Column type: FS 32025, 30\% 6-TB-2.3 di gly $\gamma$ in OV 1701, df: $0.25 \mu \mathrm{m}$, length: $20 \mathrm{~m}, 35 \mathrm{kPa}$, constant flow: $1.6 \mathrm{~mL} / \mathrm{min}$, helium; Oven Method: Initial temperature: $110{ }^{\circ} \mathrm{C}$, Initial time: $760 \mathrm{~min}$, Number of ramps: 1 , rate: $4{ }^{\circ} \mathrm{C} / \mathrm{min}$, final temperature: $210{ }^{\circ} \mathrm{C}$, hold time: 20 $\min$.

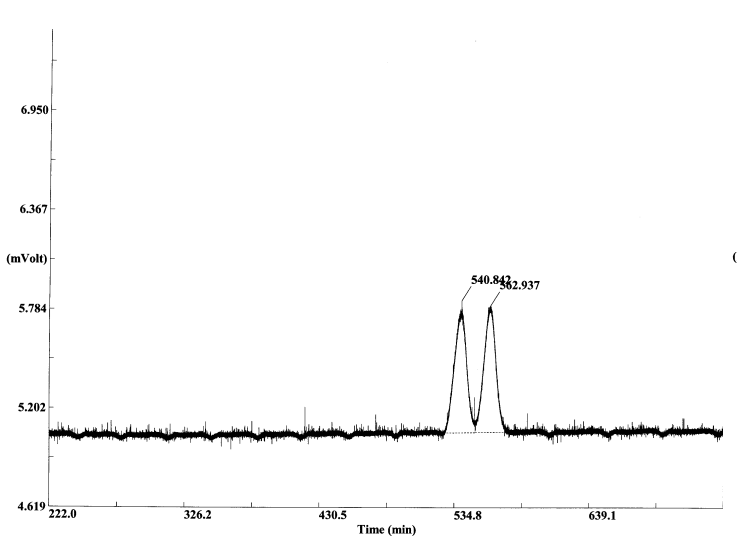

Peak \# Area \%

$1 \quad 49.9584$

Ret. Time

$2 \quad 50.0416$

540.84

562.94

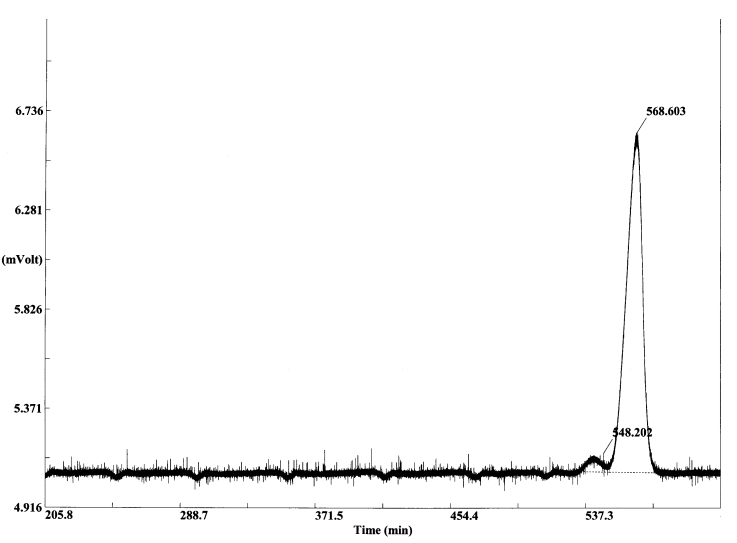

Peak \# Area \% $\quad$ Ret. Time

$1 \quad 3.6691$

548.20

$2 \quad 96.3310$

568.60

The enantiomeric purity of $(R)-5-((R)-1-(4-c h l o r o p h e n y l) e t h y l)-3-m e t h y l e n e d i h y d r o-f u r a n-2(3 H)-$ one 48 was also determined to be $92.7 \%$ by GC analysis with a chiral stationary phase column in comparison with racemic material. RT $=887.5 \mathrm{~min}$ (major isomer), $919.1 \mathrm{~min}$ (minor isomer); GCtype: Trace GC Ultra; Column type: FS 32025, 50\% 6-TB-2.3 di gly $\beta$ in PS 086, df: $0.25 \mu \mathrm{m}$, length: $20 \mathrm{~m}, 50 \mathrm{kPa}$, constant flow: $1.8 \mathrm{~mL} / \mathrm{min}$, helium; Oven Method: Initial temperature: $105^{\circ} \mathrm{C}$, Initial time: $960 \mathrm{~min}$, Number of ramps: 1, rate: $4^{\circ} \mathrm{C} / \mathrm{min}$, final temperature: $210^{\circ} \mathrm{C}$, hold time: $20 \mathrm{~min}$.
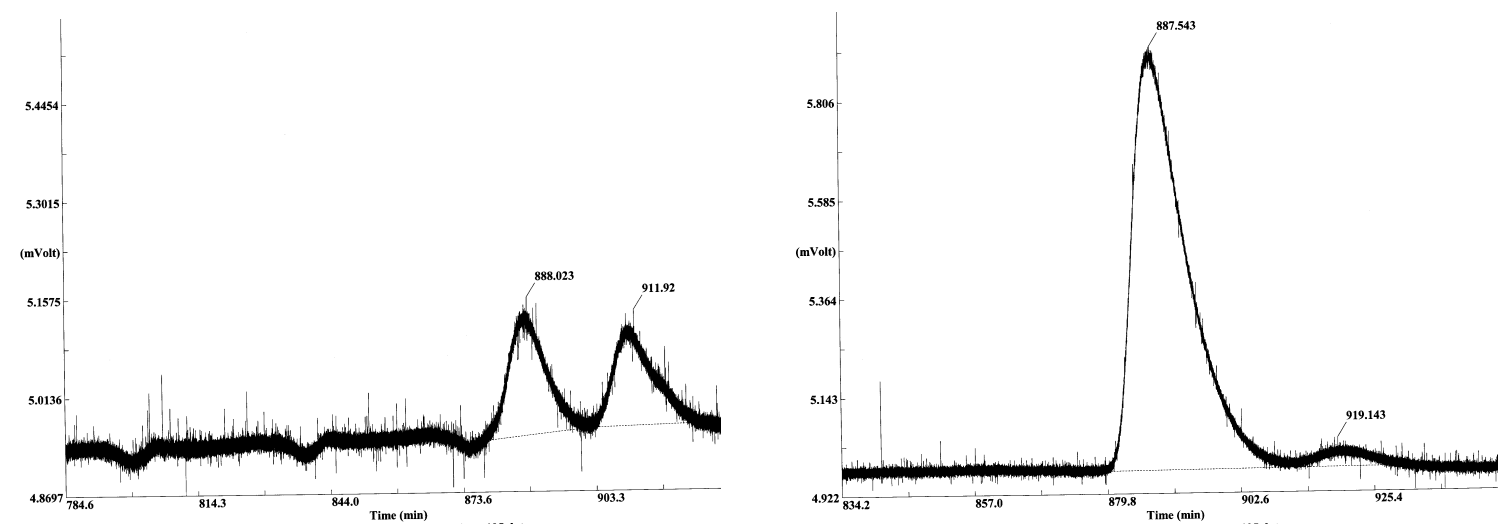

\begin{tabular}{|c|c|c|}
\hline & Area \% & Ret. Time \\
\hline 1 & 53.7721 & 888.02 \\
\hline 2 & 46.2279 & 911.92 \\
\hline
\end{tabular}

\begin{tabular}{lll}
\multicolumn{2}{l}{ Peak $\#$ Area \% } & Ret. Time \\
1 & 96.3687 & 887.54 \\
2 & 3.6313 & 91
\end{tabular}


X-Ray crystal structure of 5-([1,1'-biphenyl]-4-yl)-4-chloro-2-methylenehexyl 3,5dinitrobenzoate (syn-37)

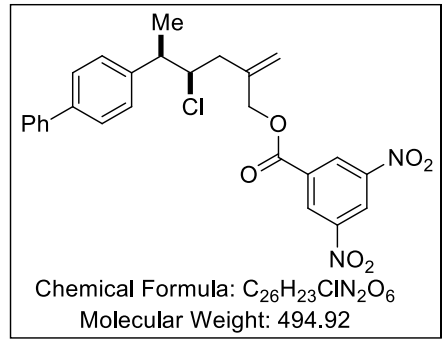

Crystal-Structure Determination. - A colorless crystal of $\mathrm{C}_{26} \mathrm{H}_{23} \mathrm{ClNO}_{6}$ was mounted in air and used for X-ray structure determination at $173 \mathrm{~K}$. All measurements were made on a Oxford Diffraction SuperNova area-detector diffractometer ${ }^{1}$ using mirror optics monochromated Mo Ka radiation $(\lambda=0.71073 \AA)$ and $\mathrm{Al}$ filtered. ${ }^{2}$ The unit cell constants and an orientation matrix for data collection were obtained from a least-squares refinement of the setting angles of reflections in the range $2.0^{\circ}<\theta<27^{\circ}$. A total of 460 frames were collected using $\omega$ scans, with $30+30$ seconds exposure time, a rotation angle of $1.0^{\circ}$ per frame and a crystal-detector distance of 65.0 $\mathrm{mm}$ at $173 \mathrm{~K}$.

Data reduction was performed using the CrysAlisPro ${ }^{1}$ program. The intensities were corrected for Lorentz and polarization effects, and an absorption correction based on the multi-scan method using SCALE3 ABSPACK in CrysAlisPro ${ }^{1}$ was applied. Data collection and refinement parameters are given in Table 1.

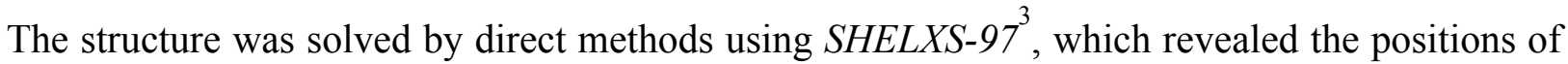
all non-hydrogen atoms of the title compound. The non-hydrogen atoms were refined anisotropically. All H-atoms were placed in geometrically calculated positions and refined using a riding model where each $\mathrm{H}$-atom was assigned a fixed isotropic displacement parameter with a value equal to $1.2 \mathrm{Ueq}$ of its parent atom (1.5Ueq for the methyl groups).

The structure is characterized by two molecules in the asymmetric unit, with very similar conformation. No symmetry relation was found between the two molecules and the hypothesis of a smaller unit cell could be safely rejected.

Refinement of the structure was carried out on $F^{2}$ using full-matrix least-squares procedures, which minimized the function $\operatorname{\Sigma w}\left(\mathrm{F}_{\mathrm{o}}{ }^{2}-\mathrm{F}_{\mathrm{c}}{ }^{2}\right)^{2}$. The weighting scheme was based on counting statistics and included a factor to downweight the intense reflections.

All calculations were performed using the SHELXL-97 $7^{3}$ program.

1) Oxford Diffraction (2010). CrysAlisPro (Version 1.171.34.44). Oxford Diffraction Ltd., Yarnton, Oxfordshire, UK.

2) Macchi, P.; Bürgi, H.B.; Chimpri, A. S.; Hauser, J.; Gál, Z. (2011) J. Appl. Cryst. 44, 763771.

3) Sheldrick, G. M. (2008). Acta Cryst. A64, 112-122. 


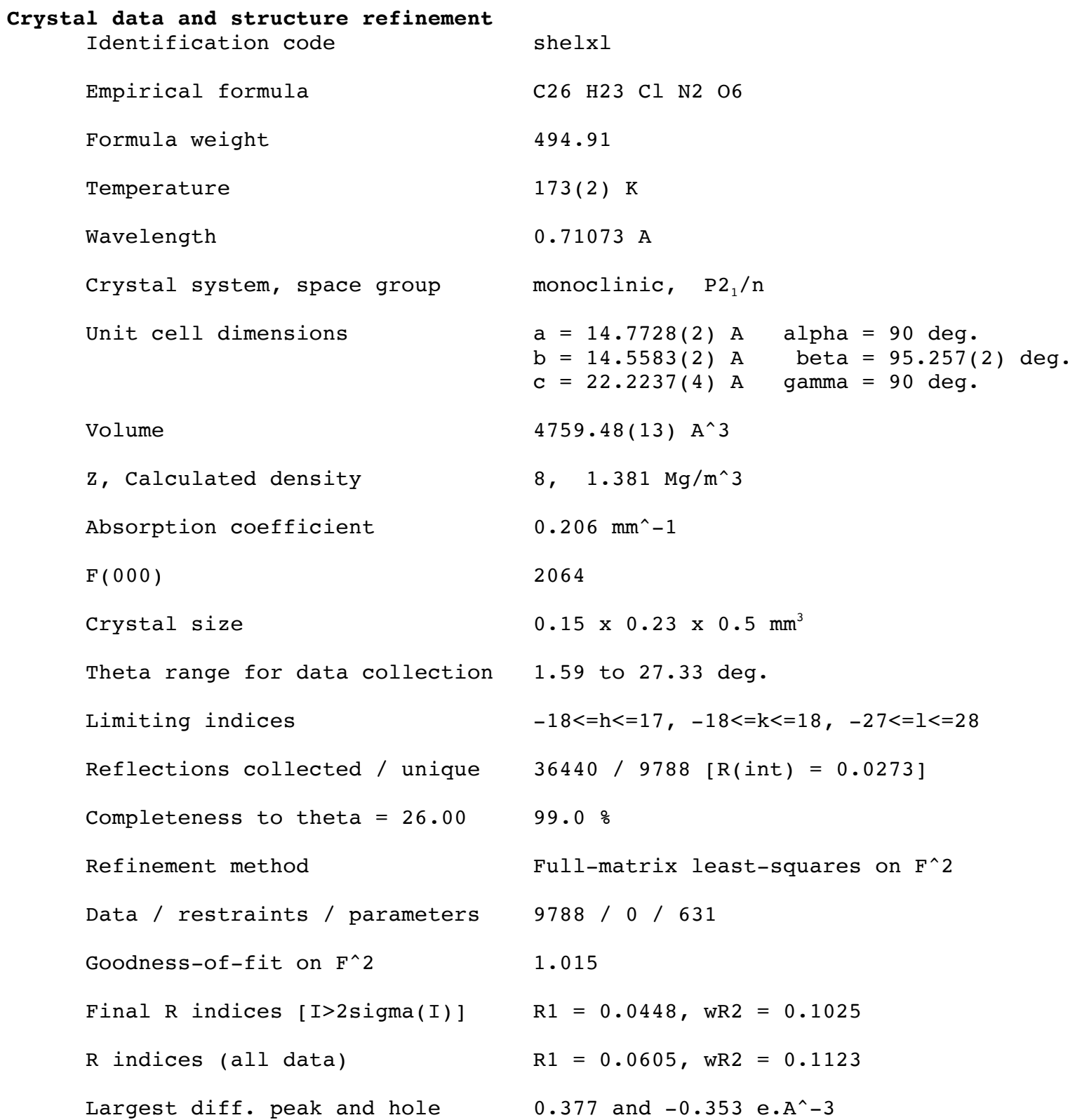


X-ray single crystal structure of syn-37 derived from the major diastereomer of 35 (50\% probability ellipsoids).

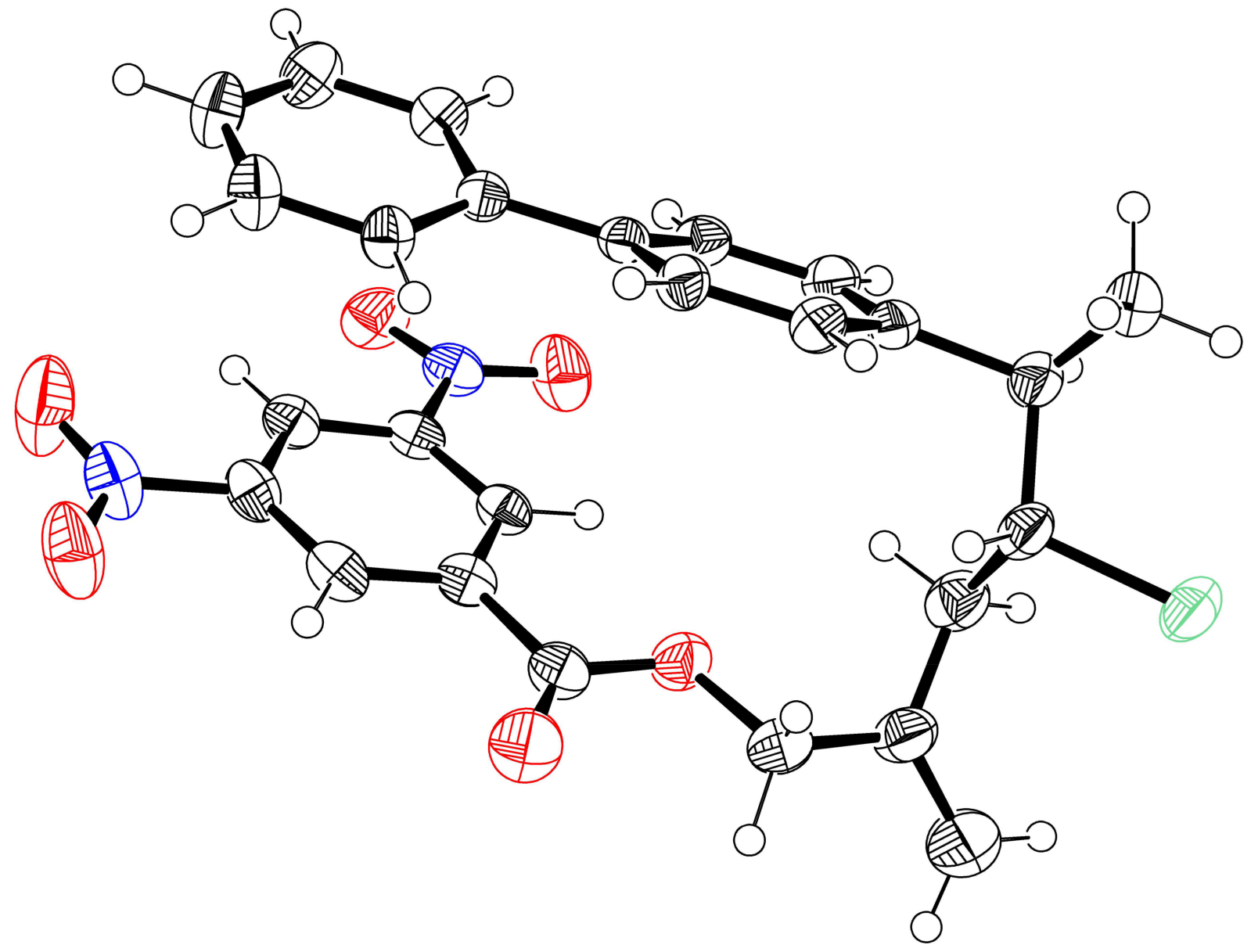




\section{$\mathrm{X}$-Ray crystal structure of 5-(4-Methoxyphenyl)-6-methyl-3-methylenetetrahydro- $2 \mathrm{H}$ - pyran-2-one 44}

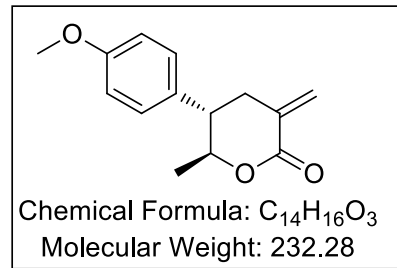

Crystal-Structure Determination. - A colorless crystal of $\mathrm{C}_{26} \mathrm{H}_{23} \mathrm{ClN}_{2} \mathrm{O}_{6}$ was mounted in air and used for X-ray structure determination at $120 \mathrm{~K}$. All measurements were made on a Oxford Diffraction SuperNova area-detector diffractometer ${ }^{1}$ using mirror optics monochromated Mo Ka radiation $(\lambda=0.71073 \AA)$ and $\mathrm{Al}$ filtered. ${ }^{2}$ The unit cell constants and an orientation matrix for data collection were obtained from a least-squares refinement of the setting angles of reflections in the range $1.5^{\circ}<\theta<27^{\circ}$. A total of 579 frames were collected using $\omega$ scans, with $40+40$ seconds exposure time, a rotation angle of $1.0^{\circ}$ per frame and a crystal-detector distance of 65.0 $\mathrm{mm}$ at $120 \mathrm{~K}$.

Data reduction was performed using the CrysAlisPro ${ }^{1}$ program. The intensities were corrected for Lorentz and polarization effects, and an absorption correction based on the multi-scan method using SCALE3 ABSPACK in CrysAlisPro ${ }^{1}$ was applied. Data collection and refinement parameters are given in Table 1.

The structure was solved by direct methods using $S H E L X S-97^{3}$, which revealed the positions of all non-hydrogen atoms of the title compound. The non-hydrogen atoms were refined anisotropically. All H-atoms were placed in geometrically calculated positions and refined using a riding model where each $\mathrm{H}$-atom was assigned a fixed isotropic displacement parameter with a value equal to $1.2 \mathrm{Ueq}$ of its parent atom (1.5Ueq for the methyl groups).

Refinement of the structure was carried out on $F^{2}$ using full-matrix least-squares procedures, which minimized the function $\operatorname{\Sigma w}\left(\mathrm{F}_{\mathrm{o}}{ }^{2}-\mathrm{F}_{\mathrm{c}}{ }^{2}\right)^{2}$. The weighting scheme was based on counting statistics and included a factor to downweight the intense reflections.

All calculations were performed using the SHELXL-97 $7^{3}$ program.

Two dynamically disordered conformations could be refined. Compared to an experiment carried out at $173 \mathrm{~K}$ (data not reported here) we conclude that cooling the sample to $120 \mathrm{~K}$ did not improve the ordering.

The presence of $\mathrm{CH}_{2} \mathrm{Cl}_{2}$ could be detected (disordered over an inversion center) and its occupancy was refined to $6 \%$. $\mathrm{H}$ atoms of the solvent were not located.

1) Oxford Diffraction (2010). CrysAlisPro (Version 1.171.34.44). Oxford Diffraction Ltd., Yarnton, Oxfordshire, UK.

2) Macchi, P.; Bürgi, H.B.; Chimpri, A. S.; Hauser, J.; Gál, Z. (2011) J. Appl. Cryst. 44, 763771 .

3) Sheldrick, G. M. (2008). Acta Cryst. A64, 112-122. 
Crystal data and structure refinement

\begin{tabular}{|c|c|}
\hline Empirical formula & $(\mathrm{C} 14 \mathrm{H} 16 \mathrm{O} 3) \quad(\mathrm{C} \mathrm{H} 2 \mathrm{Cl}) 0.06$ \\
\hline Formula weight & 237.24 \\
\hline Temperature & $120(2) \mathrm{K}$ \\
\hline Wavelength & $0.70730 \mathrm{~A}$ \\
\hline Crystal system, space group & monoclinic, $\quad \mathrm{P} 21 / \mathrm{C}$ \\
\hline Unit cell dimensions & $\begin{array}{ll}\mathrm{a}=13.2938(5) \mathrm{A} & \text { alpha }=90 \text { deg. } \\
\mathrm{b}=8.6528(3) \mathrm{A} & \text { beta }=97.224(3) \text { deg. } \\
\mathrm{c}=11.2228(3) \mathrm{A} & \text { gamma }=90 \mathrm{deg} .\end{array}$ \\
\hline Volume & $1280.70(8) \mathrm{A}^{\wedge} 3$ \\
\hline Z, Calculated density & 4, $1.230 \mathrm{Mg} / \mathrm{m}^{\wedge} 3$ \\
\hline Absorption coefficient & $0.109 \mathrm{~mm}^{\wedge}-1$ \\
\hline$F(000)$ & 505.7 \\
\hline Theta range for data collection & 1.54 to 27.11 deg. \\
\hline Limiting indices & $-16<=\mathrm{h}<=16, \quad-10<=\mathrm{k}<=11, \quad-14<=1<=14$ \\
\hline Reflections collected / unique & $10001 / 2635[R($ int $)=0.0287]$ \\
\hline Completeness to theta $=26.00$ & $99.0 \div$ \\
\hline Refinement method & Full-matrix least-squares on $\mathrm{F}^{\wedge} 2$ \\
\hline Data / restraints / parameters & $2635 / 2 / 197$ \\
\hline Goodness-of-fit on $\mathrm{F}^{\wedge} 2$ & 1.072 \\
\hline Final $R$ indices [I>2sigma(I)] & $R 1=0.0439, \quad w R 2=0.1032$ \\
\hline R indices (all data) & $\mathrm{R} 1=0.0576, \mathrm{wR} 2=0.1107$ \\
\hline Extinction coefficient & $0.0079(19)$ \\
\hline Largest diff. peak and hole & 0.263 and -0.166 e. $A^{\wedge}-3$ \\
\hline
\end{tabular}


X-ray single crystal structure of $\mathbf{4 4}$ and mechanistic rationalization for its formation (50\% probability ellipsoids).

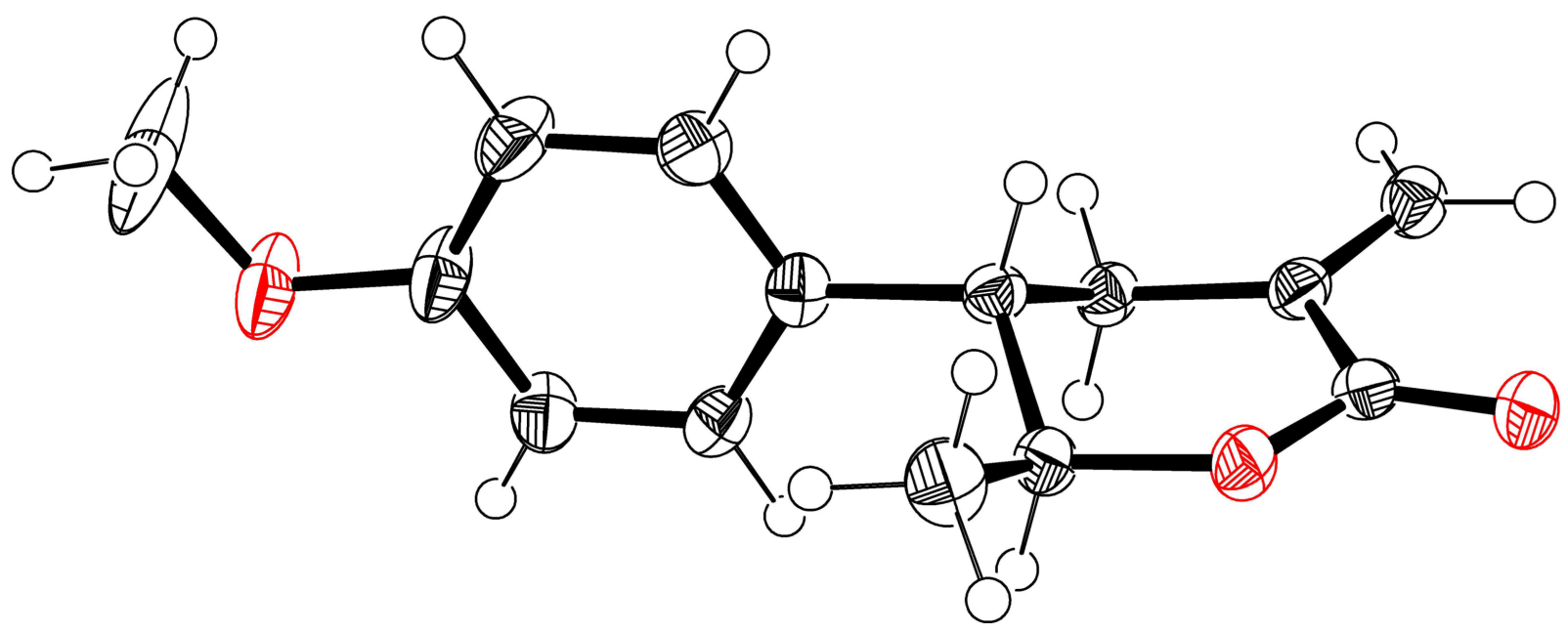




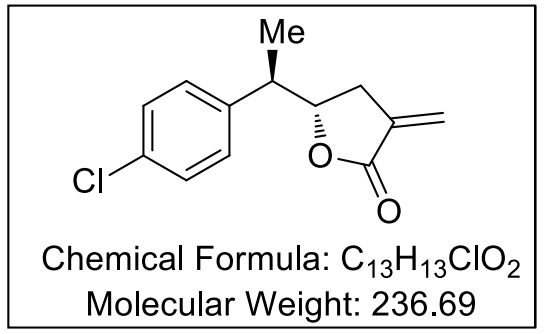

Crystal-Structure Determination. - A colorless transparent crystal of $\mathrm{C}_{13} \mathrm{H}_{13} \mathrm{Cl}_{1} \mathrm{O}_{2}$ was mounted in air and used for X-ray structure determination at ambient conditions. All measurements were made on a Oxford Diffraction SuperNova area-detector diffractometer using mirror optics monochromated Mo $K \alpha$ radiation $(\lambda=0.71073 \AA)$ and $\mathrm{Al}$ filtered. ${ }^{2}$ The unit cell constants and an orientation matrix for data collection were obtained from a least-squares refinement of the setting angles of reflections in the range $1.5^{\circ}<\theta<27.1^{\circ}$. A total of 543 frames were collected using $\omega$ scans, with $5+5$ seconds exposure time, a rotation angle of $1.0^{\circ}$ per frame, a crystal-detector distance of $65.1 \mathrm{~mm}$, at $\mathrm{T}=173(2) \mathrm{K}$.

Data reduction was performed using the CrysAlisPro ${ }^{1}$ program. The intensities were corrected for Lorentz and polarization effects, and an absorption correction based on the multi-scan method using SCALE3 ABSPACK in CrysAlisPro ${ }^{1}$ was applied. Data collection and refinement parameters are given in Table 1.

The structure was solved by direct methods using $S H E L X S-97^{3}$, which revealed the positions of all non-hydrogen atoms of the title compound. The non-hydrogen atoms were refined anisotropically. All $\mathrm{H}$-atoms were placed in geometrically calculated positions and refined using a riding model where each $\mathrm{H}$-atom was assigned a fixed isotropic displacement parameter with a value equal to $1.2 \mathrm{Ueq}$ of its parent atom.

Refinement of the structure was carried out on $F^{2}$ using full-matrix least-squares procedures, which minimized the function $\Sigma \mathrm{w}\left(\mathrm{F}_{\mathrm{o}}{ }^{2}-\mathrm{F}_{\mathrm{c}}{ }^{2}\right)^{2}$. The weighting scheme was based on counting statistics and included a factor to downweight the intense reflections. Thanks to the presence of $\mathrm{Cl}$, the absolute configuration could be determined. All calculations were performed using the SHELXL-97 $7^{2}$ program.

1) Oxford Diffraction (2010). CrysAlisPro (Version 1.171.34.44). Oxford Diffraction Ltd., Yarnton, Oxfordshire, UK.

2) Macchi, P.; Bürgi, H.B.; Chimpri, A. S.; Hauser, J.; Gál, Z. (2011) J. Appl. Cryst. 44, 763771.

3) Sheldrick, G. M. (2008). Acta Cryst. A64, 112-122. 
Crystal Data and Details of the Structure Determination

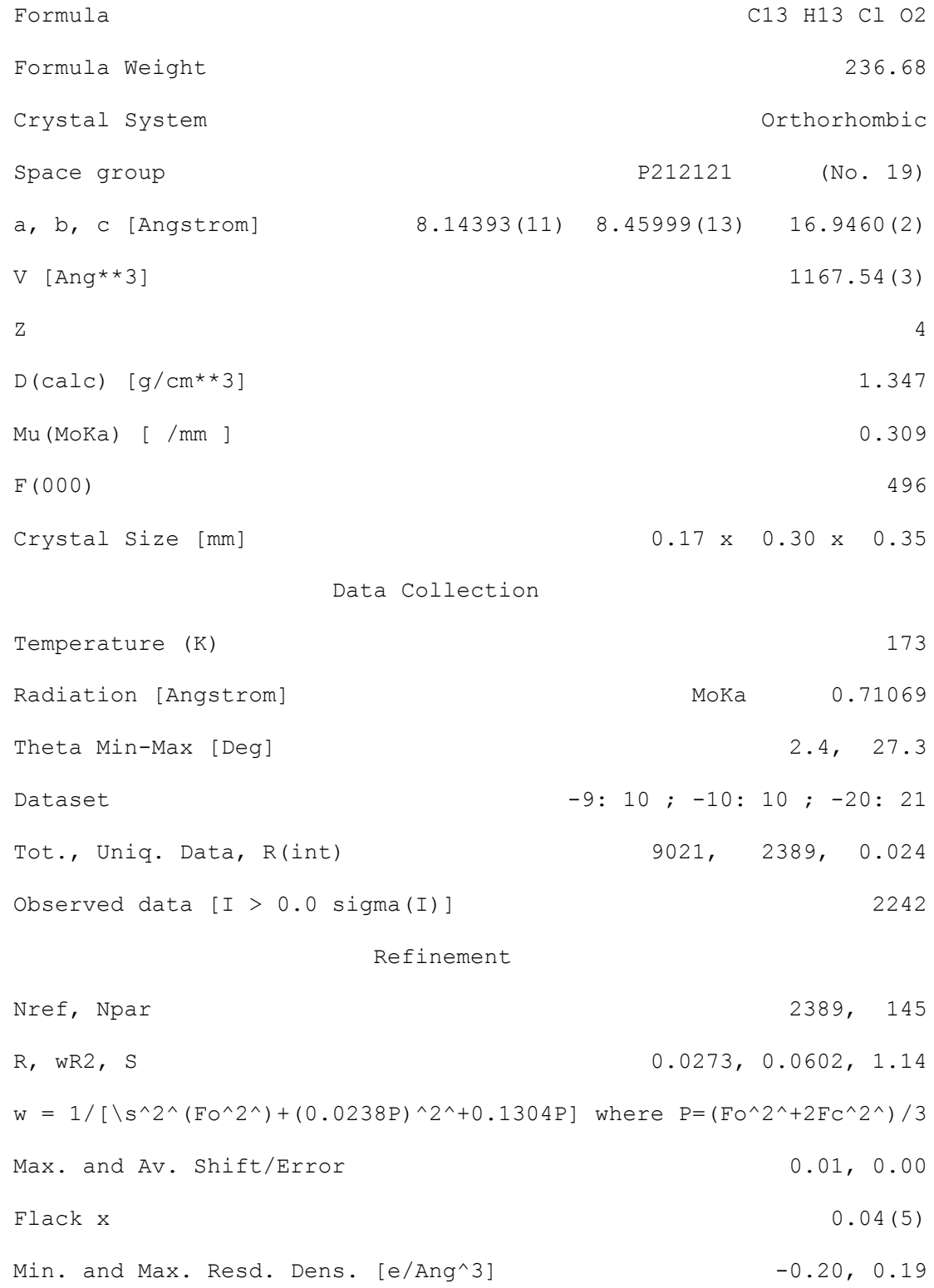

Refinement

Nref, Npar

$R$ ，WR2， $S$ $0.0273,0.0602,1.14$

$\mathrm{w}=1 /\left[\backslash \mathrm{S}^{\wedge} 2^{\wedge}\left(\mathrm{FO}^{\wedge} 2^{\wedge}\right)+(0.0238 \mathrm{P}) \wedge 2^{\wedge}+0.1304 \mathrm{P}\right]$ where $\mathrm{P}=\left(\mathrm{FO} \mathrm{O}^{\wedge} 2^{\wedge}+2 \mathrm{FC} \mathrm{C}^{\wedge} 2^{\wedge}\right) / 3$ Max. and Av. Shift/Error

$0.01,0.00$

Flack $x$

Min. and Max. Resd. Dens. [e/Ang^3] 
X-ray crystal structure of the major diastereomer $(S)-5-((R)-1-(4-c h l o r o p h e n y l) e t h y l)-3-$ methylenedihydrofuran-2(3H)-one (48) (50\% probability ellipsoids).

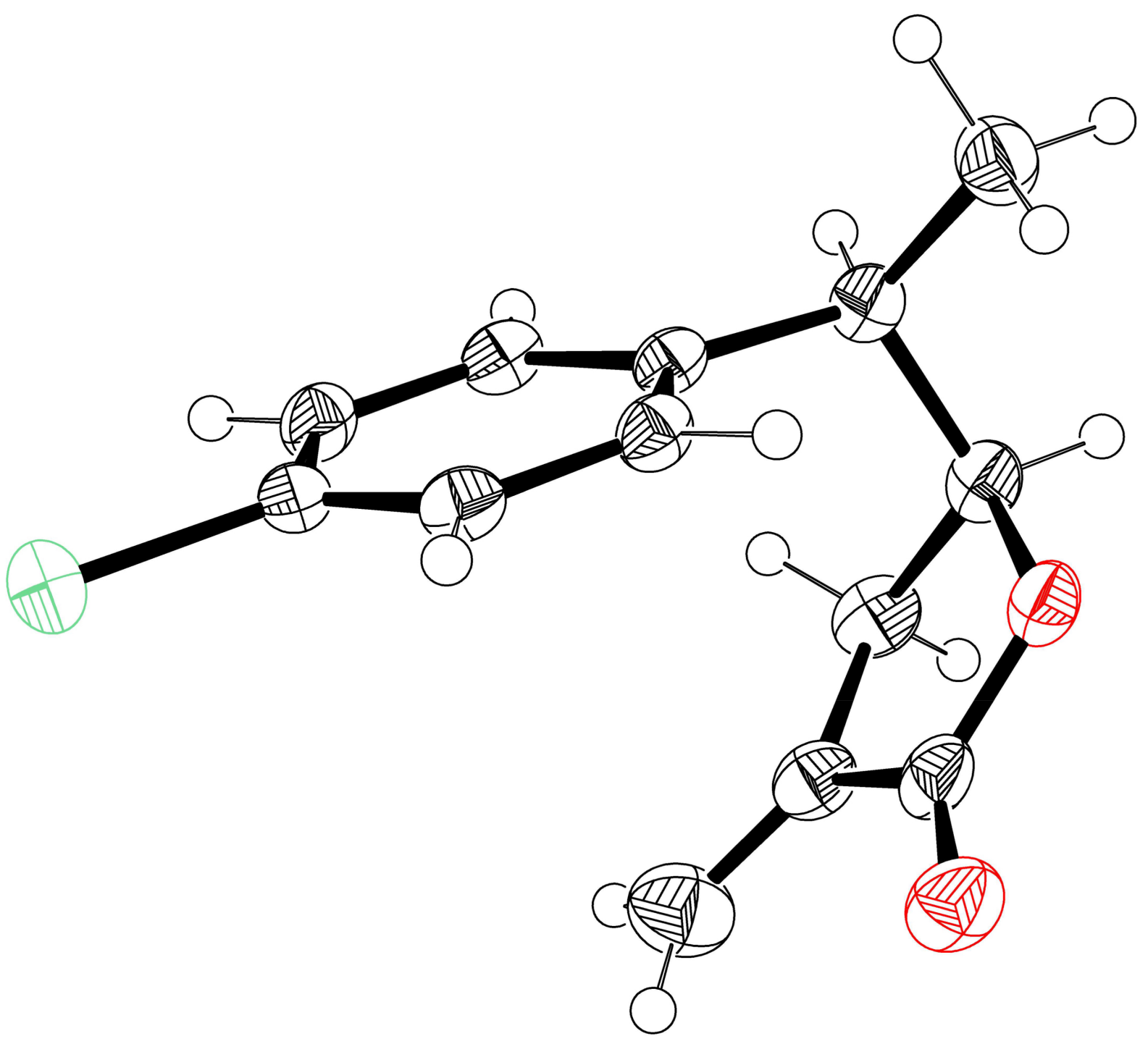

